\title{
Black Hole Based Tests of General Relativity
}

\author{
Kent Yagi \\ Department of Physics, Princeton University, Princeton, NJ 08544, USA. \\ Department of Physics, Montana State University, Bozeman, MT 59717, USA. \\ E-mail: kyagi@princeton.edu
}

\section{Leo C. Stein}

Theoretical Astrophysics, Walter Burke Institute for Theoretical Physics, California Institute of Technology, Pasadena, CA 91125, USA

Cornell Center for Astrophysics and Planetary Science (CCAPS), Cornell University, Ithaca, NY 14853, USA

\begin{abstract}
.
General relativity has passed all solar system experiments and neutron star based tests, such as binary pulsar observations, with flying colors. A more exotic arena for testing general relativity is in systems that contain one or more black holes. Black holes are the most compact objects in the universe, providing probes of the strongest-possible gravitational fields. We are motivated to study strong-field gravity since many theories give large deviations from general relativity only at large field strengths, while recovering the weak-field behavior. In this article, we review how one can probe general relativity and various alternative theories of gravity by using electromagnetic waves from a black hole with an accretion disk, and gravitational waves from black hole binaries. We first review model-independent ways of testing gravity with electromagnetic/gravitational waves from a black hole system. We then focus on selected examples of theories that extend general relativity in rather simple ways. Some important characteristics of general relativity include (but are not limited to) (i) only tensor gravitational degrees of freedom, (ii) the graviton is massless, (iii) no quadratic or higher curvatures in the action, and (iv) the theory is 4 dimensional. Altering a characteristic leads to a different extension of general relativity: (i) scalar-tensor theories, (ii) massive gravity theories, (iii) quadratic gravity, and (iv) theories with large extra dimensions. Within each theory, we describe black hole solutions, their properties, and current and projected constraints on each theory using black hole-based tests of gravity. We close this review by listing some of the open problems in model-independent tests and within each specific theory.
\end{abstract}




\section{Contents}

1 Introduction $\quad 4$

1.1 Observations . . . . . . . . . . . . . . . . . 4

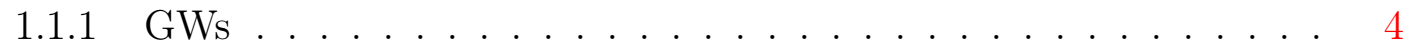

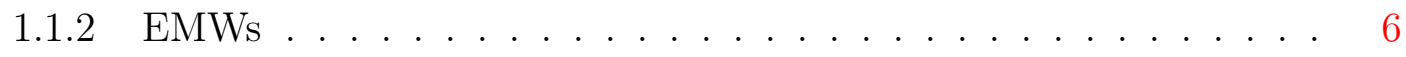

1.2 Alternative Theories of Gravity . . . . . . . . . . . . . . . 6

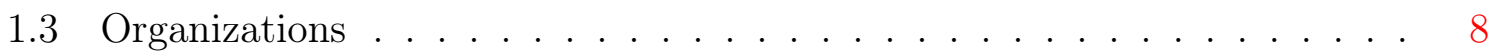

2 Theory-independent tests of GR with BHs 9

2.1 Bumpy BHs . . . . . . . . . . . . . . . . . . . 10

2.2 GW Tests . . . . . . . . . . . . . . . . . . . . 16

2.2 .1 GWs in GR . . . . . . . . . . . . . . . . 16

2.2.2 Data Analysis . . . . . . . . . . . . . . . . . . 18

2.2.3 Parameterized Tests of GR . . . . . . . . . . . . . . 19

2.2.4 BH No-hair Tests . . . . . . . . . . . . . . . . . 20

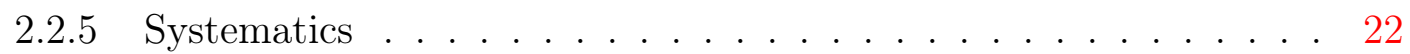

2.3 Generic Scalar Corrections to GR . . . . . . . . . . . . . . . . . 24

2.3.1 Parameterization of Theories . . . . . . . . . . . . . 25

2.3.2 Parameterization of Compact Object Properties . . . . . . . . . . 26

2.3.3 Binary, Scalar Interaction, and Pulsar Timing Bounds . . . . . . . 27

2.3.4 Parameterization of Scalar Radiation . . . . . . . . . . . . . . 29

2.3.5 Connection to PPE and Estimates of GW Bounds . . . . . . . . . 31

2.4 EMW Tests . . . . . . . . . . . . . . . . . . . . . 35

2.4.1 Continuum Spectrum . . . . . . . . . . . . . 35

2.4 .2 Iron Line Emission . . . . . . . . . . . . . . . . . . 37

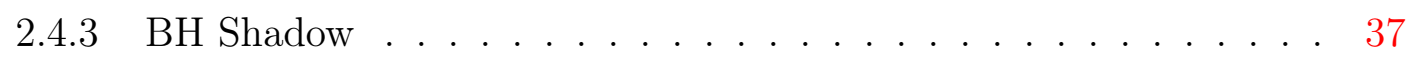

2.4.4 Quasi-periodic Oscillation . . . . . . . . . . . . 39

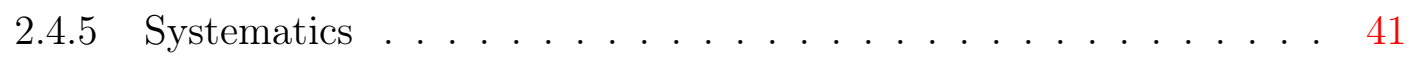

3 Scalar-Tensor Theories $\quad 42$

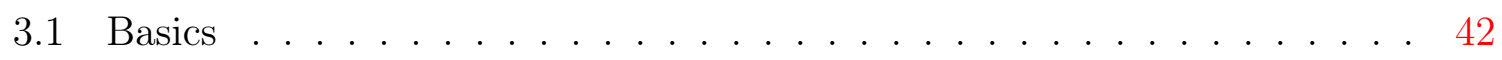

3.1.1 Theories . . . . . . . . . . . . . . . . . 43

3.1 .2 BH Solutions . . . . . . . . . . . . . . . . 44

3.1.3 Curent Constraints . . . . . . . . . . . . . . . . . . 44

3.2 GW Tests . . . . . . . . . . . . . . . . . . . . . 45

3.2.1 Massless BD Theory . . . . . . . . . . . . . . . 45

3.2 .2 Massive BD Theory . . . . . . . . . . . . . . . . 47

3.2.3 Quasi BD Theory .................. 50

3.2.4 Generic Scalar-tensor Theories . . . . . . . . . . . . . . 50

3.3 EMW Tests . . . . . . . . . . . . . . . . . . 50

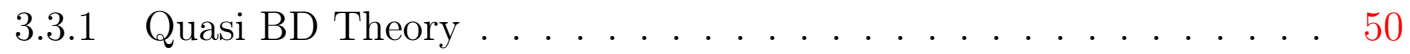

3.3.2 Generic Scalar-tensor Theories . . . . . . . . . . . . . 51 
4 Massive Gravity Theories $\quad 51$

4.1 Basics . . . . . . . . . . . . . . . . . . . 51

4.1 .1 Theories . . . . . . . . . . . . . . . . . 51

4.1 .2 Current Constraints . . . . . . . . . . . . . . . . 53

4.2 GW Tests . . . . . . . . . . . . . . . . . . 53

4.2 .1 GW Signals Alone . . . . . . . . . . . . . . . . 54

4.2 .2 Coincident Tests with EMW Signals . . . . . . . . . . . 58

5 Quadratic Gravity $\quad 59$

5.1 Basics . . . . . . . . . . . . . . . . . . . . . 59

5.2 BH Solutions . . . . . . . . . . . . . . . . . . . 61

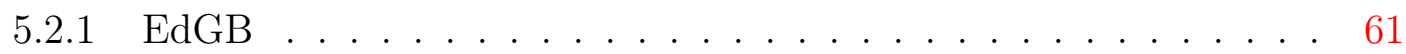

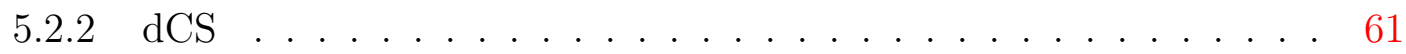

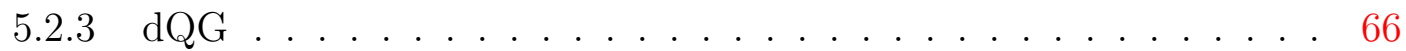

5.3 GW Tests . . . . . . . . . . . . . . . . . . . 67

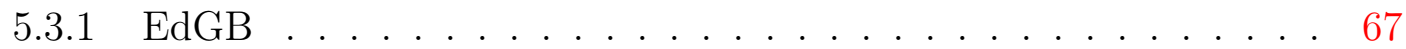

$5.3 .2 \mathrm{dCS} \ldots \ldots \ldots \ldots \ldots \ldots$

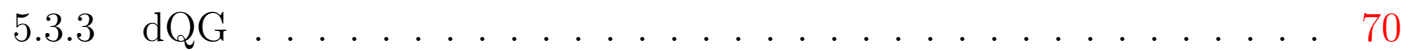

5.4 EMW Tests . . . . . . . . . . . . . . . . . . . 71

5.4 .1 EdGB . . . . . . . . . . . . . . . . . . . 71

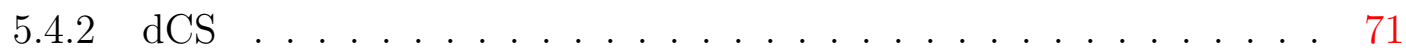

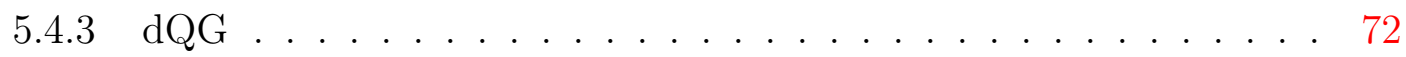

$6 \quad$ Large Extra Dimension $\quad 73$

6.1 Basics . . . . . . . . . . . . . . . . . . . . . 73

6.1.1 RS braneworld Models . . . . . . . . . . . . . . . . . . 73

6.1.2 Emparan-Fabbri-Kaloper-Tanaka Conjecture . . . . . . . . . . 75

6.2 GW Tests . . . . . . . . . . . . . . . . . . 76

6.2 .1 Monochromatic Signals . . . . . . . . . . . . . . . 77

6.2 .2 Chirping Signals . . . . . . . . . . . . . . . . . . 77

6.2 .3 Event Rate . . . . . . . . . . . . . . . . . . . . . . . . . . . 79

6.3 EMW Tests . . . . . . . . . . . . . . . . . . . 79

$\begin{array}{llr}7 & \text { Open Questions } & 80\end{array}$

7.1 Generic Ways of Testing GR . . . . . . . . . . . . . . . . . 80

7.2 Scalar-tensor Theories . . . . . . . . . . . . . . . . . . 80

7.3 Massive Gravity Theories . . . . . . . . . . . . . . . . . . . . . . 80

7.4 Quadratic Gravity . . . . . . . . . . . . . . . . . . . 81

7.5 Other Theories . . . . . . . . . . . . . . . . . . . . . 81

7.6 Common Open Problems . . . . . . . . . . . . . . . . . . . . 82 


\section{Introduction}

This past year, 2015, was the centennial anniversary of Einstein proposing general relativity (GR). So far, GR has passed all tests of gravity with flying colors. Such tests include solar system experiments [1,2], binary pulsar observations [3,4] and table-top experiments [5]. These tests are restricted to either weak-field, nondynamical or mildly dynamical situations. Precise tests of gravity in the strong-field/dynamical regime are necessary to probe certain alternative theories of gravity that can show a large deviation only in such a regime. Black holes (BHs) offer an excellent testbed to probe strong-field gravity due to their large internal gravity. BH based tests of gravity have advantages over neutron star (NS) based tests since the latter in general have large systematic errors due to uncertainties in nuclear physics (though see e.g. [6,7] that discusses how one can in principle project out such uncertainties and perform strong field tests of gravity with $\mathrm{NSs}$ ).

\subsection{Observations}

One can either use gravitational wave (GW) [8,9] or electromagnetic wave (EMW) [10,11] observations to probe strong-field gravity with BHs.

\subsubsection{GWs Regarding the former, the gravitational waveform of an inspiraling compact} binary depends on the binary's binding energy and the energy flux carried out to infinity. In alternative theories of gravity, BH solutions are in general different from the Kerr one,* which modifies the binding energy of a binary system relative to GR. Furthermore, the additional gravitational degrees of freedom (e.g. a scalar field) also affect the binding energy, and generate additional radiation, which modifies the energy flux, and in turn, the binary's evolution. One can either pick a specific theory and calculate the correction to the waveform from GR [13-43], or consider a parameterized waveform that captures deviations from GR in the waveform in a generic way [44-64]. One then carries out a matched filtering analysis, in which one takes a correlation between the GW signal and the theoretical template, to see how well one can constrain non-GR theories with future GW interferometers. One can also carry out a Bayesian model selection analysis to see whether a hypothetical observation favors GR or a specific non-GR model [61].

Another interesting way of testing GR through GWs is to probe properties of a $\mathrm{BH}$ spacetime directly. In particular, Kerr BHs enjoy the no-hair property where higher order multipole moments are completely determined by the first two, namely the $\mathrm{BH}$ mass and spin angular momentum [65-71]. After a merger, one can use the BH ringdown frequencies and damping times of different modes to check the consistency of the no-hair relation and constrain possible non-GR effects [72-90]. One can also carry out such no-hair tests with GWs from binary inspirals, such as extreme mass ratio

\footnotetext{
* Though many theories exist in which the Kerr BH is also a solution to the modified field equations [12].
} 
inspirals (EMRIs) [88,91-103]. The BH ringdown also allows us to probe the "firewall" effect [104, 105].

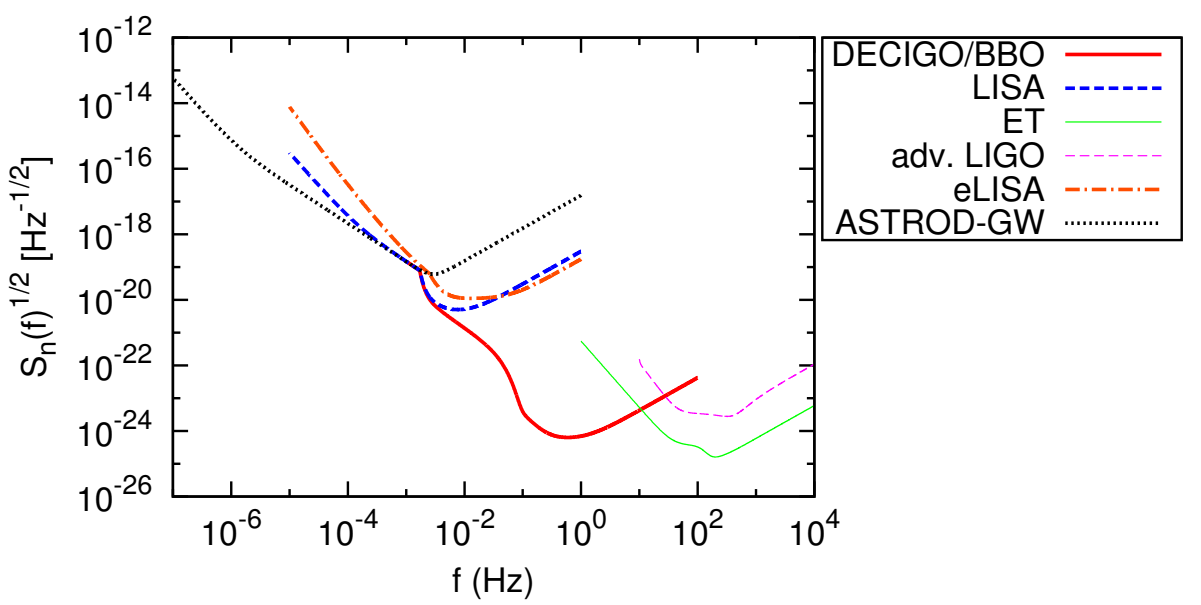

Figure 1. (Color online) Noise spectral density $S_{n}(f)$ of various GW interferometers. Adv. LIGO and ET are the second- and third-generation ground-based detectors respectively. All the other detectors are the space-borne ones. This figure is taken from [21].

Let us now describe various GW interferometers that are considered in this review. Figure 1 presents the sensitivity curve of these detectors. Currently, Adv. LIGO is in operation, and other second-generation ground-based interferometers, such as Adv. Virgo or KAGRA, will come online shortly. These detectors have armlengths of $3-4 \mathrm{~km}$ and their best sensitivities at around 100-1000Hz. The third-generation ground-based interferometer, Einstein Telescope (ET), is an underground and cryogenic detector with armlengths of $10 \mathrm{~km}$, and may be realized in the second half of the 2020-2030 decade. ET roughly has a sensitivity that is one order of magnitude better than second-generation ones. Regarding space missions, eLISA was proposed for the theme of the "Gravitational Wave Universe" for the Cosmic Vision L3 mission of the European Space Agency. The base configuration has one mother and two daughter spacecrafts separated by $10^{6} \mathrm{~km}$, and eLISA is aimed to be launched in 2034. It has its best sensitivity at around $1 \mathrm{mHz}$. DECIGO is similar to eLISA, but has four triangular constellations in total, with two of them forming a star-of-David. DECIGO has its best sensitivity at around $0.1-1 \mathrm{~Hz}$, armlengths of $10^{3} \mathrm{~km}$, and is aimed to be launched in the $2030 \mathrm{~s}$.

Astrophysical environment around BH binaries, such as accretion disks, another SMBH, magnetic fields, non-vanishing charges, etc., is unlikely to spoil strong-field tests of GR. Although thin disks around EMRIs for eLISA [104-110] and circumbinary disks around stellar-mass and intermediate-mass BH binaries for DECIGO [111] may have detectable effects, event rates are estimated to be rather small. Other non-astrophysical effects, such as cosmological constant, are also small [104,105]. Mismodelling of the waveform due to unknown self-force effects $[112,113]$ might be problematic for testing GR with EMRIs and intermediate-mass ratio inspirals. 
1.1.2. EMWs Regarding EMW observations, one can use X-ray continuum spectra [114122] and Fe K $\alpha$ line emissions [118-120,123-128] that have been used to measure BH spins in GR. Since these spectra depend strongly on the inner edge of the accretion disks, which are usually taken to be the innermost stable circular orbit (ISCO), such spectra can also be used to probe deviations from the Kerr BH. One can also use BH shadow observations at (sub-)millimeter wavelengths using very long baseline interferometry (VLBI) [119,129-145]. The size and shape of the shadow depends not only on the BH spin and inclination, but also on possible deviations from the Kerr solution. Although systematic errors are quite large, one can in principle use quasi-periodic oscillation (QPO) frequencies to probe the BH spacetime [119,146-149]. We note that although EMW tests can, in principle, probe deviations from the Kerr spacetime, this does not always mean that such tests can probe modified theories of gravity as there are many theories that allow the Kerr BH as a solution to the modified field equations (see the footnote above). On the other hand, GW tests can probe non-GR effects under the Kerr background since the perturbation is in general modified from the GR one in alternative theories of gravity, including those that admit the Kerr solution [150].

Regarding detector facilities, the continuum spectra, iron lines and QPOs have already been observed by X-ray missions, including RXTE, Chandra, NuSTAR, ASCA, Suzaku, XMM-Newton and Beppo-SAX. Future X-ray missions include Astro-H, LOFT and Athena. Astro-H is planned to be launched in 2016, while other two are aimed in the 2020s. Regarding (sub-)millimeter telescopes, Event Horizon Telescope is suitable for detecting $\mathrm{BH}$ shadows. It combines existing and planned (sub-)millimeter facilities into a high-sensitivity and high-angular-resolution telescope over the next decade.

Systematics on EMW observations for testing GR are more problematic than those in GW observations. One major origin is the correct modeling of the accretion disk. Other systematics are due to uncertainties in e.g. emissivities, hardening factors due to deviations from blackbody radiation and inclinations. QPOs also suffer from knowing the correct model a priori.

\subsection{Alternative Theories of Gravity}

In this article, we first describe model-independent ways of testing GR using GW and EMW observations as mentioned above. We then focus on testing four specific alternative theories of gravity as selected examples that extend one or more of the important characteristics of GR*, namely (i) gravity has tensor degrees of freedom, (ii) the graviton is massless, (iii) no higher order curvature terms in the action, and (iv) a 4 dimensional theory.

The first example that we consider is scalar-tensor theories [162]. These theories are one of the most well-studied alternative theories of gravity and one of the simplest extensions of GR, namely introducing scalar gravitational degrees of freedom via

* BHs can be useful for probing theories other than those mentioned in this review, e.g. Einstein-Æther theory [151-155] and string axiverse [156-161]. 
scalar fields. Such theories are motivated from the low energy effective theory of string theory [163,164], the late-time accelerated expansion of the Universe [165], and inflation [166]. As in GR, the no-hair theorems also apply to BHs in the Jordan-BransDicke type of scalar-tensor theories. Namely, stationary, asymptotically flat BHs with a single real scalar field in vacuum are the same as the Kerr one and do not possess scalar hairs [167-172]. A similar theorem holds for more generic scalar-tensor theories and $f(R)$ theories [171], and in shift-symmetric Horndeski theories [173] modulo some exceptions [174-178]. Proposed constraints on scalar-tensor theories with GWs from $\mathrm{BH} / \mathrm{NS}$ binaries have been derived in [13, 15-23, 179], while late inspiral and merger simulations of compact binaries in these theories were performed in [24, 25, 180-184]. Strong constraints on scalar-tensor theories have already been placed from binary pulsar observations [4,185-189] and solar system experiments [188,190,191]. Future BH/pulsar observations may allow us to further constrain such theories [192].

The second example is massive gravity theories, which are another well-motivated extension of GR, adding a finite mass to the graviton. At the linear level, such an extension was originally proposed by Fierz and Pauli [193], though it suffers from pathologies such as the van Dam-Veltman-Zakharov (vDVZ) discontinuity [194,195] and the Boulware-Deser ghost modes [196]. Recently, massive graviton theories have been generalized in a nonlinear way so that the theories are ghost free to all orders [197-199]. The mass of the graviton has been constrained by observations of orbits of planets in the solar system [200]. Weaker bounds on Fierz-Pauli type theory were derived from binary pulsar observations [201]. Stronger but less robust bounds were also derived from tidal interactions between galaxies [202], weak lensing [203] and galactic velocity dispersion [204]. The dispersion relation of the massive graviton modifies the phase of the gravitational waveform of compact binaries [26]. Projected constraints on the mass of the graviton with future GW interferometers have been derived in [16-21, 26-31, 33, 47, 50]. If one measures both GW signals and their EM counterparts, one can also constrain the graviton mass from the difference in the arrival time of gravitons and photons [205-207].

Our third example is quadratic gravity, which introduces generic quadratic curvature terms to the Einstein-Hilbert action that are coupled to dynamical scalar fields [176]. Such a theory includes well-known theories motivated from string theory, such as Einsteindilaton-Gauss-Bonnet (EdGB) theory [208, 209] and dynamical Chern-Simons (dCS) gravity [210-212]. BHs play a crucial role in constraining quadratic gravity. Regarding EdGB gravity, BHs possess a scalar hair [38, 174-176,178] while ordinary stars such as NSs do not [38] in the so-called decoupled limit where the scalar field linearly couples to the Gauss-Bonnet density in the action. This means that binary pulsar observations with two NSs cannot place stringent constraints on this theory due to the absence of the scalar dipole radiation, while $\mathrm{BH}$ binaries allow us to place strong constraints on the theory with future radio and GW observations [38,213,214]. Regarding dCS gravity, spherically symmetric solutions are the same as in GR due to parity considerations. Therefore, one needs to consider spinning compact objects in order to place meaningful constraints on the theory. Since BHs generally spin faster than NSs, BHs in general place 
stronger constraints on dCS gravity than NSs. For example, future GW interferometers, such as Adv. LIGO, may place constraints that are six orders of magnitude stronger than the current solar system and table-top experiments [39].

Our fourth example is a large extra dimension model, in particular the braneworld model proposed by Randall and Sundrum [215] (RS-II model), in which we live on a 4-dimensional brane with a positive tension and the bulk is anti-de Sitter (AdS) spacetime. The size of the extra dimension is characterized by the AdS curvature length $\ell$. Gravity is localized on the brane and the gravitational potential acquires a correction that is proportional to $\ell^{2}$ [215,216], which has been constrained from table-top experiments [217]. Emparan et al. [218] and Tanaka [219] applied the anti-de Sitter/conformal field theory (AdS/CFT) correspondence to brane-localized BHs, where the correspondence states that the gravity in the $A d S_{5} \times S^{5}$ spacetime can be interpreted as the four-dimensional $\mathcal{N}=4$ $U(N)$ super Yang-Mills theory on the AdS boundary [220,221]. They proposed that such brane-localized BHs effectively lose their mass classically due to the universal acceleration in the bulk away from the brane [222] and the Gregory-Laflamme instability [222]. On the CFT side, such an effect can be understood as an enhanced Hawking radiation due to the large number of degrees of freedom in CFT [221]. Such an enhanced Hawking radiation has been constrained from astrophysical BH observations, in particular, the orbital decay rate of low-mass X-ray binaries (LMXBs)* with BHs [223, 224]. Proposed constraints with GW observations on the size of extra dimension are derived in [42,43]. Notice that BHs are crucial to probe such an effect since it is absent for ordinary stars like NSs.

Since static brane-localized BH solutions have now been constructed numerically in [225, 226], the correctness of the conjecture proposed in [218, 219] is unclear. Nevertheless, we cover this theory in this review for the following reasons: (i) the conjecture itself is interesting and may stimulate other work in the context of the AdS/CFT correspondence; (ii) the gravitational waveform for BH binaries with the mass loss effect can have other applications, such as to varying $G$ theories [227] and to the BH binary system with dark energy accretion [228, 229]; and (iii) the RS-II model serves as an interesting example to see how GWs in higher-dimensional theories are modified from those in GR.

\subsection{Organizations}

The organization of this article is as follows. In Sec. 2, we review how one can carry out model-independent tests of GR with BHs. We first explain bumpy BH spacetimes that parameterize deviations away from the Kerr spacetime in a generic manner. We then summarize how to perform model-independent tests using GWs and EMWs from a system that contains at least one BH. We then turn to the four specific types of modifications

* A LMXB consists of a NS or a BH as a primary and a companion being e.g. a post-main sequence star. Gas from the companion fills its Roche lobe and accretes onto the primary. X-rays are emitted from the accretion disk around the primary, and in the case of an NS primary, also from the NS surface. 
discussed above. In Secs. 3-6, we review (respectively) scalar-tensor theories, massive gravity theories, quadratic gravity, and extra dimension theories. In each section, we describe BH solutions within each theory and their properties. Then, we explain current and projected constraints with GWs and EMWs in each theory. We conclude in Sec. 7 by giving some of the open questions related to testing GR with BHs generically, in the specific theories we have discussed, and in theories beyond the scope of this review. We mostly use geometrical units with $c=1=G$.

\section{Theory-independent tests of GR with BHs}

Scientists give many names to their approaches to experimentally testing a physical theory $X$, but in essence there are just two methods. First, there are the theory-specific tests, and second, there are the theory-independent tests. There are advantages and disadvantages to each approach. Theory-specific tests (sometimes called "external" tests) naturally require specific alternative physical theories $Y_{i}$ which compete with $X$ at giving a more faithful mathematical description of nature. Meanwhile, theory-independent tests (sometimes called "internal" or "null" tests) focus on whether or not observations agree with the predictions of theory $X$, without regard to specific alternatives.

The advantage of a theory-specific test is that it is straightforward to quantify whether theory $X$ or $Y_{i}$ is a more faithful description of nature. This quantification comes from Bayesian model comparison [61]. Model comparison is easy to understand: it quantifies the degree of belief you should place in theory $X$ over theory $Y_{i}$, given some prior beliefs, and observational/experimental data whose signatures may be computed in each theory. The disadvantage is that you need to have a specific calculation in each theory under consideration, of e.g. the spectrum of an accretion disk, or the precession of an orbit, or a gravitational waveform. Each theory $Y_{i}$ is different and thus requires unique calculations which can not be reused for theory $Y_{j \neq i}$.

This same argument is an advantage for theory-independent tests. In theoryindependent tests, one tries to parametrize just enough of the physics to create parametric tests of some experiment or observation. Examples are to parametrize the spacetime metric (the parameterized post-Newtonian or PPN formalism [1,2, 230]), parametrize orbital post-Keplerian (PK) parameters [3, 231-233], parametrize perturbations about the FLRW space (the parameterized post-Friedmann (PPF) framework [234-236]), or parametrize a gravitational waveform (the parameterized post-Einstein or PPE framework $[9,46])$. However, it is difficult to create a parametrization that is sufficiently general that it captures the signatures of all alternative theories. For example, the original PPE framework failed to capture the GW signature in dCS gravity, where the leading deformation from GR is sourced by spin effects. Similarly, many bumpy BH spacetimes (which we discuss in Sec. 2.1) fail to capture BH solutions in deformations of GR.

In this Section we turn our attention to theory-independent tests of GR with BHs. In Sec. 2.1 we will discuss parametrizing the spacetime around a BH. In Sec. 2.2 we 
will focus on parameterized gravitational wave tests, including the parameterized postEinstein framework. In Sec. 2.3 we will discuss a quasi-theory-independent framework for parametrizing scalar theories which deform away from GR. In Sec. 2.4 we will discuss electromagnetic observations of BHs which can be used for theory-independent and theory-specific tests.

\subsection{Bumpy BHs}

One of the most astounding predictions of classical GR is that of BHs - regions of spacetime where gravity is so strong that light, matter, and any information which enters may never exit. This is perhaps the most non-Newtonian phenomenon in general relativity, and exists because GR is nonlinear (while Newtonian gravity is linear).* Nonlinearity is essential because a gravitational field may become so strong that gravity itself gravitates - thus a BH consists of nothing besides gravity. In fact, though real astrophysical BHs are formed from the collapse of a massive star, once a BH forms, it very rapidly settles down [238] to the unique vacuum solution in GR: the Kerr metric. The BH has no "memory" of any light, matter, or information that falls in, save for the total mass $M$ and spin angular momentum $S$. BHs in GR are thus very simple, forming a two-parameter family of spacetimes, with all of the (Geroch-Hansen) multipole moments given by the simple formula [70, 239, 240]

$$
M_{\ell}+i S_{\ell}=M(i a)^{\ell}
$$

where $a \equiv S / M,-M \leq a \leq M$ is the Kerr spin parameter.

An obvious theory-independent test of GR is to ask: Are real astrophysical BHs described by the Kerr metric? This is sometimes called the Kerr hypothesis.

One may pose several analogous theory-independent questions in different contexts of testing GR. As mentioned above, the parameterized post-Newtonian, post-Keplerian, and parameterized post-Einstein frameworks are such examples. Let us look at the weakfield limit of GR, where tests of GR are addressed by the parameterized post-Newtonian (PPN) formalism [1,2]. There, the metric may be parameterized in standard harmonic coordinates via [1]

$$
\begin{aligned}
& g_{00}=-1+2 U-2 \beta U^{2}+\ldots \\
& g_{j k}=(1+2 \gamma U) \delta_{j k}+\ldots
\end{aligned}
$$

where $U$ is the potential sourced by mass density, and $\beta, \gamma$ are two of the ten PPN parameters. PPN is specially tailored to the weak-field regime where the metric is close to the flat Minkowski metric. PPN is perturbative, and in order for it to be applicable, the potential $U \sim G M / r c^{2}$ must be small compared to 1 . This condition is obviously violated in extreme spacetimes such as those harboring NSs and BHs, which have dimensionless compactnesses $M_{\star} / R_{\star} \sim 0.1-1$.

* Michell and Laplace developed a black-hole-like concept that predates Maxwell theory, treating light as corpuscular [237]. 
Therefore a different approach is needed to formulate a null test of the Kerr hypothesis. The approach we discuss here is to parametrize BH metrics or deviations away from the Kerr metric. To parametrize the weak-field metric up to 1PN order requires only 10 numbers, the $\operatorname{PPN}$ parameters $\left(\gamma, \beta, \xi, \alpha_{1,2,3}, \zeta_{1,2,3,4}\right)$. In contrast, an infinite number of parameters are required to specify deviations from the Kerr spacetime. In fact, it is still unknown if the parameter space of interest is countably infinite or if it is uncountable. Parameterized deviations from the Kerr metric are commonly referred to as bumpy BHs.

The starting point for most bumpy BH investigations is the Kerr metric, which in Boyer-Lindquist coordinates $(t, r, \theta, \phi)$ reads [241]

$$
\begin{aligned}
d s^{2}= & -\left(1-\frac{2 M r}{\Sigma}\right) d t^{2}-\frac{4 M a r \sin ^{2} \theta}{\Sigma} d t d \phi+\frac{\Sigma}{\Delta} d r^{2}+\Sigma d \theta^{2} \\
& +\left(r^{2}+a^{2}+\frac{2 M a^{2} r \sin ^{2} \theta}{\Sigma}\right) d \phi^{2}
\end{aligned}
$$

with the usual definitions $\Sigma \equiv r^{2}+a^{2} \cos ^{2} \theta$ and $\Delta \equiv r^{2}-2 M r+a^{2}=\left(r-r_{+}\right)\left(r-r_{-}\right)$, which has the two roots of the equation $\Delta=0$ at $r_{ \pm} \equiv M \pm \sqrt{M^{2}-a^{2}}$. These roots are the locations of null surfaces of constant $r$ which coincide with outer $(+)$ and inner $(-)$ horizons.

The Kerr metric is a special case of a stationary, axisymmetric, asymptotically flat (SAAF) spacetime, which is the expectation for an isolated $\mathrm{BH}$ geometry at late times, after perturbations have settled down. The general form for a SAAF spacetime is given in cylindrical coordinates $(t, \phi, \rho, z)$ as [241],

$$
d s^{2}=-V(d t-w d \phi)^{2}+V^{-1} \rho^{2} d \phi^{2}+\Omega^{2}\left(d \rho^{2}+\Lambda d z^{2}\right)
$$

where $(V, w, \Omega, \Lambda)$ are functions of $(\rho, z)$ only. This form requires certain integrability conditions to be satisfied (see [241] for details); these are automatically satisfied when working in vacuum in GR. They may also be satisfied in other theories of gravity, but a deeper analysis would be required. When using Ricci-flatness, Eq. (5) can be further simplified to the Weyl-Lewis-Papapetrou form,

$$
d s^{2}=-V(d t-w d \phi)^{2}+V^{-1}\left[\rho^{2} d \phi^{2}+e^{2 \gamma}\left(d \rho^{2}+d z^{2}\right)\right]
$$

where $\gamma=\frac{1}{2} \ln \left(V \Omega^{2}\right)$.

The solutions for functions $(V, w, \gamma)$ needed to express the Kerr metric can be found in Eq. (51) of ref. [242]. Of course this solution enjoys the multipole relation given in Eq. (1), and the Kerr metric is said to have "no hairs" (though two hairs would be more appropriate). Other solutions for $(V, w, \gamma)$ will have different multipole moments (the Geroch-Hansen multipole moments [70,240] are defined for stationary spacetimes via derivatives of analytic metric functions taken at the point at spatial infinity). This generalization was carried out by Manko and Novikov [243] and can describe an exterior vacuum spacetime with arbitrary mass multipole moments. This same spirit was taken 
up more recently by Backdahl [244-246], paying special attention to when a prescribed set of multipole moments leads to the existence of a solution. Essentially, one can not choose arbitrary multipole moments for $\left(M_{\ell}, S_{\ell}\right)$; in order for a solution to exist, the choice of multipole moments must satisfy a convergence criterion.

It is not enough to simply describe the metric of a bumpy BH spacetime. To test the Kerr hypothesis, we also need gauge-invariant observables to compare between models and data. The first such calculations were performed by Ryan [91]. Ryan considered the motion of a small compact object in the field of a large bumpy $\mathrm{BH}$, leaving $(V, w, \gamma)$ free, focusing on nearly-circular and nearly-equatorial trajectories. Ryan treated the motion as being geodesic except for back-reaction.

Let us comment here on the applicability of the geodesic motion assumption, because many papers on bumpy BHs include geodesic calculations. The assumption of geodesic motion restricts one to bodies in theories of gravity which respect the strong equivalence principle (SEP). Note that the SEP is violated in a large number of theories, e.g. dCS and EdGB. In Jordan-Brans-Dicke, BHs in the absence of a scalar field will respect the SEP, while NSs violate the SEP. If the degree of violation is sufficiently small then the geodesic approximation may still be relevant.

Within this framework, Ryan computed the motion, calculated precession frequencies and GWs in the adiabatic approximation, and gave a prescription for finding the unknown multipole moments from the observables. Later, Ryan performed a Fisher matrix forecast of how well LISA would be able to extract multipole moments [92].

One criticism of the preceding work is that it is tied to the Geroch-Hansen multipoles, which are evaluated at the point at spatial infinity. This is a Newtonian-inspired formalism, but it might not be relevant in the strong-field region, close to the horizon. Indeed, some of the bumpy black hole spacetimes we discuss have naked singularities, lack horizons, or have other pathologies [247]. A different approach was taken in [248-250]. Collins and Hughes [248] advocated to instead apply perturbation theory about the Schwarzschild solution. That is, we take the metric functions to be

$$
(V, w, \gamma)=\left(V_{\text {Schw }}, w_{\text {Schw }}, \gamma_{\text {schw }}\right)+\epsilon\left(V^{(1)}, w^{(1)}, \gamma^{(1)}\right)+\mathcal{O}\left(\epsilon^{2}\right)
$$

and expand the Einstein field equations to linear order in $\epsilon$. In principle, this approach should be equivalent to the linearization of the approach used by Manko, Novikov, Ryan, and Backdahl, at least in the far-field.

For two examples of bumpy solutions, Collins and Hughes took as sources (i) point masses along the North and South poles of the coordinate system (inspired by [251]), and (ii) adding an axisymmetric equatorial ring of mass to the spacetime. Both of these perturbations affect the Geroch-Hansen quadrupole (and higher moments) of the spacetime. For each of these perturbations, Collins and Hughes then computed (geodesic) orbital frequencies, which could in principle be measured through pulsar timing or by a LISA-like mission.

This same approach was extended by Vigeland and Hughes (VH) [249] and Vigeland [250]. Rather than using the artificial point or ring sources of [248], VH 
found everywhere-Ricci-flat solutions to order $\mathcal{O}(\epsilon)$ for a number of low multipole orders. For purely mass multipole moments, the VH approach should be equivalent to a MankoNovikov solution [243] near Schwarzschild, with the higher mass moments perturbatively small. In the Schwarzschild background, VH's axially-symmetric solutions are all of the qualitative form

$$
\gamma_{\ell}^{(1)} \sim B_{\ell} M^{\ell+1} \frac{P_{\ell}\left(\cos \theta_{\text {Weyl }}\right)}{\left(\rho^{2}+z^{2}\right)^{(\ell+1) / 2}}
$$

for some angular mode number $\ell$, where $\cos \theta_{\text {Weyl }}=z / \sqrt{\rho^{2}+z^{2}}$, and where $B_{\ell}$ is a dimensionless coefficient which describes how large the $\ell$-mode bump is. For these solutions they then went on to compute the (geodesic) trajectories of orbits, including finding the excess pericenter precession induced by the bumpy modes.

Further, VH went on to use the Newman-Janis trick [252] to perform a complex rotation on the Schwarzschild solution in an attempt to construct bumpy rotating solutions.* They also computed (geodesic) orbits and observables, such as the three precession frequencies of an orbit, for the rotating bumpy metrics with $\ell=2,3,4$.

Vigeland further extended this line of work in [250]. Firstly, she generalized the types of bumps which could be produced from only the mass-type moments in [249] to both the mass- and spin-type moments. Secondly, she constructed the mapping between these linearized bumps and the Geroch-Hansen multipole moments. In particular, she found that a linearized perturbation of order $\ell$ only affected Geroch-Hansen multipole moments of order $\ell$ and above.

Another simple and commonly-used parameterization is the quasi-Kerr metric of Glampedakis and Babak [95]. Unlike the previously-mentioned parameterizations, the quasi-Kerr metric only has one additional parameter instead of an infinite number. Furthermore, this free parameter is designed to affect the quadrupole (though we are unaware of general $\ell$ Geroch-Hansen moments being computed for the quasi-Kerr metric, so it likely affects higher moments). The principle behind constructing the quasi-Kerr metric was to extract the quadrupole and spin-squared pieces of the Hartle-Thorne slow rotation metric [254], which describes the exterior spacetime of any slowly-rotating body. The difference between the Hartle-Thorne metric for general quadrupole moment (not tied to the spin, like in Kerr) and that for the Kerr solution then gives a metric deformation to "paste onto" the Kerr metric. Glampedakis and Babak also studied (geodesic) motion in the quasi-Kerr metric, especially for equatorial orbits, computing "kludge" gravitational waveforms. $\dagger$

* Applying the NJ transformation to the non-rotating VH spacetime leads to a spacetime which is not Ricci-flat. The applicability of the NJ trick has been called into question by Hansen and Yunes [253], especially in constructing metrics which are supposed to satisfy field equations other than those of GR.

$\dagger$ A "kludge" approach is one which mixes different approximations, not necessarily arising from a single consistent approximation scheme. For example: using particles on Kerr geodesics as sources entering into PN waveform formulas; or using GR waveform formulas even in the context of other theories. Kludge waveforms are easier to compute and should still capture the qualitative nature of full waveforms. 
Many of the observational properties of Glampedakis and Babak's proposed quasi-Kerr metric have been computed by Johannsen and Psaltis in a series of papers $[123,130,146,255]$. This series of papers focused on (i): general properties, including the location of the horizon and ISCO, redshifts, and null trajectories; (ii): numerically ray-tracing images of the quasi-Kerr spacetime (assuming that photons still follow null geodesics); (iii): computing geodesic and epicyclic (precession) frequencies in the strong-field, which could be putative QPO frequencies; and (iv): simulating the $\mathrm{Fe}-\mathrm{K} \alpha$ line spectrum from the strong-field region of the quasi-Kerr spacetime, which includes relativistic broadening and boosting (again assuming that photons and matter follow respectively null and timelike geodesics).

Johannsen and Psaltis also introduced another proposal called the modified Kerr metric [256]. Their approach was to start with a spherically symmetric and static metric,

$$
d s^{2}=-f(r)[1+h(r)] d t^{2}+f(r)^{-1}[1+h(r)] d r^{2}+r^{2}\left(d \theta^{2}+\sin ^{2} \theta d \phi^{2}\right),
$$

where $f(r)=1-2 M / r$ is the usual Schwarzschild metric function. In the limit of $h(r) \rightarrow 0$ this metric agrees with Schwarzschild. For $h(r)$ they take the ansatz

$$
h(r)=\sum_{k=0}^{\infty} \epsilon_{k}\left(\frac{M}{r}\right)^{k}
$$

which is inspired by a far-field expansion. Johannsen and Psaltis then arrive at the modified Kerr metric by applying the Newman-Janis trick [252], as was earlier done by $\mathrm{VH}$ [249]. As mentioned in the footnote on the previous page, applying the NJ trick is very questionable here, especially since Eq. (9) is not a solution to the Einstein equations [253]. Johannsen and Psaltis then go on to find the event horizon, compute circular, equatorial geodesics, and find the ISCO and circular, equatorial photon orbit (again assuming photons follow null geodesics).

Cardoso, Pani, and Rico performed a thorough analysis and extended the Johannsen and Psaltis metric in [257]. Their generalization simply introduced $h^{t}$ and $h^{r}$ in place of $h$ in the $t t$ and $r r$ components in Eq. (9), doubling the infinite number of coefficients needed to parameterize the spacetime. They also showed that all coefficients become equally important in the strong-field, near the central object. This is a strong criticism of the Glampedakis and Babak metric, which only has one parameter. This same feature highlights a severe degeneracy in strong-field observables in terms of the $\epsilon_{k}$ parameters. Furthermore, Cardoso, Pani, and Rico also showed that known non-GR solutions, such as those in dCS and EdGB, do not fit within either the original or the extended modified Kerr parameterization. For these reasons, the authors urged extreme caution with this parameterization.

More recently, Rezzolla and Zhidenko took a similarly-inspired yet unique approach to parameterizing deviations from Schwarzschild [258]. Their starting point was to write the spherically-symmetric, static metric as

$$
d s^{2}=-N^{2}(r) d t^{2}+\frac{B^{2}(r)}{N^{2}(r)} d r^{2}+r^{2}\left(d \theta^{2}+\sin ^{2} \theta d \phi^{2}\right) .
$$


At this point it is essentially the same as the starting point of Johannsen and Psaltis. However, to maintain regularity at the horizon and to parameterize the near-horizon behavior, Rezzolla and Zhidenko use the coordinate $x=1-r_{0} / r$ (where $N\left(r_{0}\right)=0$ is the coordinate of the horizon), and write

$$
N^{2}=x A(x), \quad A(x)>0, \quad 0 \leq x \leq 1 .
$$

They then expand $A(x)$ and $B(x)$ as

$$
\begin{aligned}
& A(x)=1-\epsilon(1-x)+\left(a_{0}-\epsilon\right)(1-x)^{2}+\tilde{A}(x)(1-x)^{3}, \\
& B(x)=1+b_{0}(1-x)+\tilde{B}(x)(1-x)^{2} .
\end{aligned}
$$

The functions $\tilde{A}, \tilde{B}$ are then expanded as infinite continued fractions,

$$
\tilde{A}(x)=\frac{a_{1}}{1+\frac{a_{2} x}{1+\frac{a_{3} x}{1+\ldots}}}, \quad \tilde{B}(x)=\frac{b_{1}}{1+\frac{b_{2} x}{1+\frac{b_{3} x}{1+\ldots}}} .
$$

In Eqs. (13)-(15), $\epsilon, a_{n}$ and $b_{n}$ (with $n$ a non-negative integer) are real constants. The continued fraction expansion may have desirable convergence properties. Rezzolla and Zhidenko showed how to map between their metric and the non-rotating one of Johannsen and Psaltis. They computed the ISCO and circular photon orbit (again assuming that test bodies and photons move on timelike and null geodesics, respectively), and found the quasinormal mode frequencies for a test scalar field living on this background spacetime. However, these frequencies may have little relation to the quasinormal modes of the spacetime itself, which depends on the dynamics in the theory of gravity which gives rise to these solutions. Different theories could give rise to the same solution but have distinct quasinormal frequencies. Finally, Rezzolla and Zhidenko considered a Hartle-Thorne-like slow-rotation expansion to linear order in spin to additionally parameterize spinning objects.

Some of the aforementioned approaches to bumpy BHs satisfied the Einstein field equations; some used the NJ trick; and some were either ad-hoc or general parameterizations of SAAF spacetimes. A completely different approach was taken in Vigeland, Yunes, and Stein [242] (VYS). The authors were motivated to find spacetimes which retained the Liouville integrability of test-particle motion that the Kerr spacetime enjoys. VYS did start with a totally general perturbative parameterization by linearizing the Weyl-Lewis-Papapetrou metric about the Kerr solution. From this point, VYS then imposed the condition that the perturbed spacetime retained a Carter-like second rank Killing tensor to leading order in perturbation theory, which restricts the function space of deformations to Kerr. This space is still large enough that it was possible to describe the slowly-rotating $\mathrm{BH}$ solution in $\mathrm{dCS}$ [259] (it was later found that at order $\mathcal{O}\left(a^{2}\right)$, the dCS BH does not have a third integral of motion, and thus does not fall under the VYS 
parameterization [260]). The metric of VYS was later simplified in [261] and reads

$$
\begin{aligned}
g_{t t} & =-\frac{\tilde{\Sigma}\left[\Delta-a^{2} A_{2}(r)^{2} \sin ^{2} \theta\right]}{\left[\left(r^{2}+a^{2}\right) A_{1}(r)-a^{2} A_{2}(r) \sin ^{2} \theta\right]^{2}}, \\
g_{t \phi} & =-\frac{a\left[\left(r^{2}+a^{2}\right) A_{1}(r) A_{2}(r)-\Delta\right] \tilde{\Sigma} \sin ^{2} \theta}{\left[\left(r^{2}+a^{2}\right) A_{1}(r)-a^{2} A_{2}(r) \sin ^{2} \theta\right]^{2}}, \\
g_{r r} & =\frac{\tilde{\Sigma}}{\Delta A_{5}(r)}, \\
g_{\theta \theta} & =\tilde{\Sigma} \\
g_{\phi \phi} & =\frac{\tilde{\Sigma} \sin ^{2} \theta\left[\left(r^{2}+a^{2}\right)^{2} A_{1}(r)^{2}-a^{2} \Delta \sin ^{2} \theta\right]}{\left[\left(r^{2}+a^{2}\right) A_{1}(r)-a^{2} A_{2}(r) \sin ^{2} \theta\right]^{2}},
\end{aligned}
$$

where Johannsen defined $\tilde{\Sigma} \equiv \Sigma+f(r)$. This metric has four functional degrees of freedom, $A_{1,2,5}(r)$ and $f(r)$. Still, this metric is of limited applicability because there is no reason that the $\mathrm{BH}$ solution in some theory of gravity should have Liouville integrable geodesics (for instance the $\mathcal{O}\left(a^{2}\right) \mathrm{BH}$ in dCS).

Despite this plethora of bumpy BH frameworks, none of them are completely satisfactory. Most of them have pathologies, some of which were investigated in [247]. Johannsen showed that some of these metrics include naked singularities and closed timelike curves. In addition, no one framework seems to be able to capture all BH solutions from non-GR theories. More research is required along this line of work to reach a theoretically satisfactory description of bumpy BHs and the phenomena which take place in these spacetimes.

\section{2. $G W$ Tests}

In this subsection, we review how one can perform a model independent tests of GR with GW observations. We refer to recent reviews [8,9] for more details on this topic.

2.2.1. GWs in GR Let us first briefly explain how one can calculate GWs from a compact binary inspiral with masses $\left(m_{1}, m_{2}\right)$ and a separation $r_{12}$ to leading postNewtonian (PN) order, where one expands in $m / r_{12}$ with $m=m_{1}+m_{2}$ representing the total mass. Such a PN approximation corresponds to assuming that the velocity of binary constituents is much smaller than the speed of light. The total energy of the system is given by $E=-\eta m^{2} /\left(2 r_{12}\right)$ while the energy flux emitted from this binary is calculated from the quadrupole formula as $\dot{E}=-(32 / 5) \eta^{2} m^{5} / r_{12}^{5}$, where $\eta \equiv m_{1} m_{2} / m^{2}$ is the symmetric mass ratio. Since GW frequency $f$ is related to the orbital angular frequency $\Omega=\sqrt{m / r_{12}^{3}}$ by $f=\Omega / \pi$, one can derive the evolution of the GW frequency as

$$
\dot{f}=\frac{d f}{d r_{12}} \frac{d r_{12}}{d E} \frac{d E}{d t}=\frac{96}{5} \pi^{8 / 3} \mathcal{M}^{5 / 3} f^{11 / 3},
$$


where $\mathcal{M} \equiv m \eta^{3 / 5}$ is the chirp mass. One solves this equation to yield

$$
f=\left(\frac{5}{256}\right)^{3 / 8} \frac{1}{\pi \mathcal{M}^{5 / 8}} \frac{1}{\left(t_{0}-t\right)^{3 / 8}}, \quad \phi(t)=\int 2 \pi f d t=-2\left(\frac{1}{5} \mathcal{M}^{-1}\left(t_{0}-t\right)\right)^{5 / 8}+\phi_{0}
$$

where $\phi(t)$ is the GW phase in the time domain and $t_{0}$ and $\phi_{0}$ correspond to the time and phase at coalescence respectively.

Next, we derive the gravitational waveform in the Fourier domain by applying the stationary phase approximation (see e.g. [262]). Let us begin by Fourier transform a function $B(t) \equiv A(t) \cos \phi(t)$ as

$$
\begin{aligned}
\tilde{B}(f) & \equiv \int_{-\infty}^{\infty} A(t) \cos \phi(t) e^{2 \pi i f t} d t \\
& =\frac{1}{2} \int_{-\infty}^{\infty} A(t)\left(e^{i \phi(t)}+e^{-i \phi(t)}\right) e^{2 \pi i f t} d t .
\end{aligned}
$$

Since $f>0$ and $d \phi / d t>0$, the term proportional to $e^{i(2 \pi f t-\phi)}$ has a stationary point while the term proportional to $e^{i(2 \pi f t+\phi)}$ oscillates rapidly and becomes negligible upon integration. Requiring $d \ln A / d t \ll d \phi / d t$, the stationary point $t_{*}(f)$ is determined by solving $2 \pi f=d \phi\left(t_{*}\right) / d t$, while one can approximate $A(t)$ with $A\left(t_{*}\right)$ and take it out of the integral. Expanding the exponent in the integrand around $t=t_{*}$, one finds

$$
\tilde{B}(f) \approx \frac{1}{\sqrt{2}} A\left(t_{*}\right)\left(\frac{d^{2} \phi\left(t_{*}\right)}{d t^{2}}\right)^{-1 / 2} e^{i\left[2 \pi f t_{*}-\phi\left(t_{*}\right)\right]} \int_{-\infty}^{\infty} e^{-i X^{2}} d X
$$

with $X \equiv \sqrt{\left(d^{2} \phi\left(t_{*}\right) / d t^{2}\right) / 2}\left(t-t_{*}\right)$. Using the Fresnel integral $\int_{-\infty}^{\infty} e^{-i X^{2}} d X=\sqrt{\pi} e^{-i \pi / 4}$, one finally arrives at

$$
\tilde{B}(f) \approx \frac{1}{2} A\left(t_{*}\right)\left(\frac{d f\left(t_{*}\right)}{d t}\right)^{-1 / 2} e^{i\left[2 \pi f t_{*}-\phi(f)-\pi / 4\right]},
$$

where we remind that $t_{*}=t_{*}(f)$.

Let us now apply this result to the gravitational waveform. From Eq. (21), one finds that the phase in the Fourier domain is $2 \pi f t_{*}(f)-\phi\left[t_{*}(f)\right]-\pi / 4$. On the other hand, from Eq. (18), one finds

$$
t_{*}(f)=t_{0}-5 \mathcal{M}(8 \pi \mathcal{M} f)^{-8 / 3}, \quad \phi(f)=\phi_{0}-2(8 \pi \mathcal{M} f)^{-5 / 3} .
$$

Combining Eqs. (21) and (22), the phase of the gravitational waveform in GR is given by

$$
\Psi_{\mathrm{GR}}(f)=2 \pi f t_{0}-\phi_{0}-\frac{\pi}{4}+\frac{3}{128}(\pi \mathcal{M} f)^{-5 / 3}\left\{1+\mathcal{O}\left[(\pi \mathcal{M} f)^{2 / 3}\right]\right\} .
$$

One can extend the above calculations by including higher PN corrections. For example, for a non-spinning binary, the waveform phase is known up to 3.5PN order [263] and are given in the form

$$
\Psi_{\mathrm{GR}}(f)=2 \pi f t_{0}-\phi_{0}-\frac{\pi}{4}+\sum_{i=0}^{7}\left[\psi_{i}^{(0)}+\psi_{i}^{(1)} \ln f\right] f^{(i-5) / 3}
$$

where the coefficients $\psi_{i}^{(0)}$ and $\psi_{i}^{(1)}$ are functions of $m$ and $\eta$. 
2.2.2. Data Analysis We here review how one can carry out a GW data analysis to probe strong-field gravity with GWs. One method is to use a Fisher analysis [264,265] and determine how well future GW interferometers can measure parameters that characterize deviations away from GR. Such an analysis corresponds to performing a matched filtering analysis and take correlations between GW signals and templates. Fisher analysis is only valid when the signal-to-noise ratio (SNR) is large.

Let us start by assuming that the detector noise is stationary and Gaussian. Then, the noise follows a Gaussian probability distribution given by

$$
p\left(n_{0}\right) \propto \exp \left[-\frac{1}{2}\left(n_{0} \mid n_{0}\right)\right], \quad(A \mid B) \equiv 4 \operatorname{Re} \int_{0}^{\infty} d f \frac{\tilde{A}^{*}(f) \tilde{B}(f)}{S_{n}(f)}
$$

where $S_{n}(f)$ is the noise power spectral density (see Fig. 1). SNR is defined via this definition of the inner product as $\rho=\sqrt{(h \mid h)}$, where $h$ is the GW signal. We denote the detected signal $s(t)=h\left(t ; \boldsymbol{\theta}_{t}\right)+n_{0}(t)$, where $\boldsymbol{\theta}_{t}$ is the true parameters of a binary. Then, one can rewrite Eq. (25) as

$$
p\left(\boldsymbol{\theta}_{t} \mid s\right) \propto \exp \left[\left(h_{t} \mid s\right)-\frac{1}{2}\left(h_{t} \mid h_{t}\right)\right],
$$

with $h_{t} \equiv h\left(\boldsymbol{\theta}_{t}\right)$. Determined binary parameters $\hat{\boldsymbol{\theta}}$ are those that maximize $p\left(\boldsymbol{\theta}_{t} \mid s\right)$, which satisfies $\left(\partial_{i} h_{t} \mid s\right)-\left(\partial_{i} h_{t} \mid h_{t}\right)=0$ with $\partial_{i} \equiv \partial / \partial \theta_{t}^{i}$. Introducing $\Delta \theta^{i}$ by $\theta_{t}^{i}=\hat{\theta}^{i}+\Delta \theta^{i}$ and expanding Eq. (26) around $\Delta \theta^{i}=0$ and keeping up to quadratic order, one finds

$$
p(\boldsymbol{\theta} \mid s) \propto \exp \left[-\frac{1}{2} \Gamma_{i j} \Delta \theta^{i} \Delta \theta^{j}\right],
$$

where $\Gamma_{i j}=\left(\partial_{i} \partial_{j} h \mid h-s\right)+\left(\partial_{i} h \mid \partial_{j} h\right)$ is the Fisher matrix. Since $h-s=-n$, one can neglect the first term of $\Gamma_{i j}$ above in the large SNR limit and finds

$$
\Gamma_{i j}=\left(\partial_{i} h \mid \partial_{j} h\right)
$$

The measurement error of $\theta^{i}$ then becomes

$$
\sqrt{\left\langle\left(\Delta \theta^{i}\right)^{2}\right\rangle}=\sqrt{\left(\Gamma^{-1}\right)_{i i}}
$$

A more sophisticated way of carrying out a parameter estimation study is to perform a Bayesian Markov Chain Monte Carlo (MCMC) analysis [266,267]. With this approach, one can directly calculate the posterior distribution $p(\boldsymbol{\theta} \mid d, \overline{\mathcal{M}})$ of a parameter for a given data $d$ within a model $\overline{\mathcal{M}}$ through the Bayes' theorem as

$$
p(\boldsymbol{\theta} \mid d, \overline{\mathcal{M}})=\frac{p(d \mid \boldsymbol{\theta}, \overline{\mathcal{M}}) p(\boldsymbol{\theta}, \overline{\mathcal{M}})}{p(d, \overline{\mathcal{M}})} .
$$

Here, $p(d \mid \boldsymbol{\theta}, \overline{\mathcal{M}})$ and $p(\boldsymbol{\theta}, \overline{\mathcal{M}})$ are the likelihood and prior distributions respectively, while $p(d, \overline{\mathcal{M}})$ is the evidence given by

$$
p(d, \overline{\mathcal{M}})=\int d \boldsymbol{\theta} p(d \mid \boldsymbol{\theta}, \overline{\mathcal{M}}) p(\boldsymbol{\theta}, \overline{\mathcal{M}}) .
$$


One can also carry out a model selection study between models $\overline{\mathcal{M}}_{1}$ and $\overline{\mathcal{M}}_{2}$ by calculating the Bayes factor defined by taking the ratio of the evidence between the two models. Such a Bayes factor can be calculated with e.g. thermodynamic integration [267, 268], nested sampling [268] and reverse jump, MCMC [266]. References [47,51] proposed a less computationally expensive way of calculating the Bayes factor between GR and non-GR models, whose validity was confirmed in [63].

2.2.3. Parameterized Tests of GR We will next review how one can construct parameterized gravitational waveforms from compact binaries to perform strong-field tests of gravity. The first approach extends the PN waveform in Eq. (24) to non-GR theories, which allows us to carry out tests of gravity similar to parameterized postKeplerian (PPK) tests with binary pulsar observations [3, 4, 189]. PPK parameters depend on two masses of the binary constituents. This means that any two independent measurement of two PPK parameters allows us to determine two masses, while an additional PPK parameter measurement enables us to perform a consistency test of GR.

Arun et al. [44] proposed that since $\psi_{i}^{(0)}$ and $\psi_{i}^{(1)}$ in Eq. (24) for gravitational waveforms from a compact binary inspiral also depend on two masses of the binary constituents, independent measurement of such PN parameters allows us to perform a consistency test of GR. The authors treated all of the coefficients with different is independently and found that large degeneracies exist among these parameters with a LISA observation. The authors extended this analysis in $[45,53]$ by treating $\psi_{0}^{(0)}$ and $\psi_{2}^{(0)}$ as fundamental parameters to determine two masses, and consider one of the remaining parameters as an additional parameter to perform consistency tests of GR. The authors used the restricted PN waveforms in [45] and the full PN waveform in [53], where the former only keeps the leading PN order term in the amplitude, while the latter keeps up to 3PN order. In particular, Ref. [53] studied how well one can constrain deviations from GR by detecting GWs from BH binaries with future ground-based GW interferometers, such as Adv. LIGO and ET, with a Fisher analysis. The authors found that one can carry out such a type of tests with Adv. LIGO using $\psi_{3}^{(0)}$ as an additional parameter, while one can also use other parameters with ET. Such a Fisher analysis was extended in $[49,61]$ to a Bayesian model selection study for binary NSs using a GW data analysis pipeline to test GR, called Test Infrastructure for GEneral Relativity (TIGER).

One of the limitations in the above approach is that one can only consider corrections to non-vanishing terms in the waveform phase in GR. This means that one cannot capture e.g. the effect of scalar radiation entering at $-1 \mathrm{PN}$ relative to GR and non-GR corrections to the waveform amplitude. In order to construct a more generic parameterized waveform, Yunes and Pretorius [46] proposed a parameterized post-Einsteinian (PPE) waveform. They first introduce a generic parameterized correction to the binding energy (or Kepler's law) and energy flux. Then, the authors propagate such corrections to the waveform of a compact binary inspiral in the Fourier domain and found the simplest PPE waveform given by

$$
\tilde{h}(f)=A_{\mathrm{GR}}(f)\left(1+\alpha_{\mathrm{ppE}} u^{a_{\mathrm{pPE}}}\right) \exp \left[i \Psi_{\mathrm{GR}}(f)+\beta_{\mathrm{ppE}} u^{b_{\mathrm{pPE}}}\right],
$$


where $u \equiv \pi \mathcal{M} f, A_{\mathrm{GR}}$ and $\Psi_{\mathrm{GR}}$ are the waveform amplitude and phase in GR respectively, while $\alpha_{\mathrm{ppE}}, a_{\mathrm{ppE}}, \beta_{\mathrm{ppE}}$ and $b_{\mathrm{ppE}}$ are the PPE parameters that encodes the dominant deviations from GR in the amplitude and phase. The waveform reduces to that in GR when $\alpha_{\mathrm{ppE}}=0=\beta_{\mathrm{ppE}}$ and the correction terms in the amplitude and phase correspond to $(3 / 2) a_{\mathrm{ppE}} \mathrm{PN}$ and $\left[(3 / 2) b_{\mathrm{ppE}}+5 / 2\right] \mathrm{PN}$ corrections relative to GR respectively. Such a parameterization can capture gravitational waveforms in e.g. scalar-tensor theories, massive gravity theories, quadratic gravity (including EdGB and dCS gravity), variable $G$ theories, large extra dimension models, Lorentz violating theories (including EinsteinÆther and khronometric gravity). The authors also constructed PPE waveforms in the merger and ringdown phases.

Many follow-up papers were published that extends the original PPE framework. Cornish et al. [47] performed a Bayesian inference and model selection study to reveal how well future GW interferometers, such as Adv. LIGO and LISA, can measure PPE parameters $\alpha_{\mathrm{ppE}}$ and $\beta_{\mathrm{ppE}}$ for each $a_{\mathrm{ppE}}$ and $b_{\mathrm{ppE}}$ respectively. The authors compared their results with [269], where the latter studies the current constraints on $\beta_{\mathrm{ppE}}$ for each $b_{\mathrm{ppE}}$ from orbital decay rate of the double binary pulsar J0737-3039 [270-273], and found that Adv. LIGO will perform better than such binary pulsar observations for $b_{\mathrm{ppE}} \geq-1.5$. Chatziioannou et al. [54] extended Eq. (32) to include non-tensorial polarization modes. Sampson et al. [56] included subdominant PPE correction terms to the waveform in Eq. (32) and carried out a Bayesian inference study. They found that the simplest PPE waveform in Eq. (32) is sufficient for measuring non-GR effects. Reference [58] relates the PPN framework for solar system experiments and the PPK framework for binary pulsar observations to the PPE one. Sampson et al. [57] studied the "trouble with templates" and found following: (i) LIGO/VIRGO network may miss signals using GR templates if the true signal is the non-GR one that is still consistent with existing constraints. (ii) The simplest PPE model of Eq. (32) can still capture non-GR signals that suddenly shows deviations from GR at a certain frequency, such as dynamical scalarization in quasi BD theory [180-184] and massive scalar tensor theories [22,188]. (iii) If one uses Eq. (32) that only contains the inspiral phase to filter the actual signals that also contain merger and ringdown phases, the deviations from GR may be misidentified. Loutrel et al. [64] constructed a non-GR, parameterized burst signals of GWs from binaries with highly eccentric orbits. Huwler et al. [62] constructed a PPE waveform in the time domain. Vallisneri and Yunes [55] and Vitale and Del Pozzo [60] carried out a Bayesian analysis and studied stealth bias [47] in detail, which refers to deviations from GR that are too small to be detected but causes systematic errors that are larger than statistical errors. They found that such systematic errors (in particular, in the mass measurements) can be significant even within the current bounds. However, detailed analyses on systematic errors on PPE parameters themselves due to the waveform mismodeling (similar to those carried out in GR in e.g. [104,105]) are still missing.

2.2.4. BH No-hair Tests We now review testing BH no-hair relations among multipole moments with (i) inspiral and (ii) ringdown GWs. Regarding the former, most literature 
considers EMRIs as a smaller compact object orbits around the central larger $\mathrm{BH}$ many times (since the radiation reaction is suppressed by the mass ratio compared to comparable mass binaries), and hence it can probe the central BH spacetime directly. As mentioned in Sec. 2.1, Ryan [91,92] derived a PN gravitational waveform from EMRIs with arbitrary multipole moments of the central object and carried out a Fisher analysis. He found that if LISA detects GW signals from an EMRI with masses $\left(10,10^{5}\right) M_{\odot}$ and SNR of 100, the dimensionless quadrupole moment can be measured to $\sim 10^{-3}$. Barack and Cutler [94] improved Ryan's analysis by considering generic orbits and including the satellite's motion. They constructed the waveform using an analytic-kludge approach [274], where the authors model, at every instant, the orbit as Newtonian, emitting the leading order GWs, and solve the PN equations to secularly evolve the orbit's parameters. They found constraints that are better than Ryan's.

One can also study future prospects of testing the BH no-hair property with GWs by studying test particle motion around a bumpy metric (see Sec. 2.1 for a detailed explanation of bumpy metrics). Glampedakis and Babak [95] studied geodesic motion of a test particle around a quasi-Kerr object, which was constructed based on the HartleThorne metric [254,275]. They then constructed gravitational waveforms and found that the mismatch from the Kerr waveform can be significant for a modest deviation from Kerr, suggesting that using the Kerr waveform templates for extracting GWs from EMRIs around a non-Kerr metric may result in a significant loss of SNRs. Gair et al. [96] studied orbits around a metric proposed by Manko and Novikov (MN) [243], whose multipole moments differ from the Kerr ones for $\ell \geq 2$. They found that certain orbits lead to an ergodic motion due to the loss of the Carter-like constant. Orbital properties around the MN spacetime were more thoroughly studied by Apostolatos, Lukes-Gerakopoulos and Contopoulos [97-99]. According to the Poincaré-Birkhoff theorem, the resonant tori in the phase space of a perturbed integrable system disintegrate to form a chain of islands, inside which the ratio of fundamental frequencies stays constant (also frequencies themselves evolve). The appearance of such islands is a distinct feature of non-Kerr spacetime, and hence, a plateau in the evolution of the ratio of the fundamental frequencies, when the orbit crosses the island, is a smoking gun for a generic deviations from Kerr. Gair and Yunes [101] constructed EMRI waveforms from the VYS bumpy metric [242] using an analytic-kludge approach.

Yet, another approach is to study GWs from EMRIs of exotic compact objects. Kesden et al. [93] studied the orbital motion of a compact object around a supermassive boson stars (see Ref. [276] for a review on boson stars) and found that a stable orbit exists even inside the surface of a boson star. They evolved the trajectory of a compact object from the exterior to the interior of the central boson star and found that GWs emitted from such a system is distinguishable from those from EMRIs with a central BH. Macedo et al. $[88,103]$ extended the above analysis by calculating the gravitational and scalar radiation in a consistent and fully relativistic way for a few different boson star models. They found that due to the absence of the event horizon, resonant oscillation modes are resonantly excited by orbiting compact objects and GW signals at the last 
stage of the inspiral becomes qualitatively different from that of an EMRI with a central BH. GWs from merging boson stars were studied in [277,278]. On the other hand,EMRI waveforms of gravastars [279] were calculated in [100], where gravastars have the de Sitter and Schwarzschild metric in the interior and exterior respectively, with a thin-shell with a tensions that stitches these two metrics. Similar to the boson star case, EMRI waveforms for gravastars have peaks that correspond to resonant modes excited during the inspiral phase by the resonant scattering of GWs due to the gravastar's surface. For a gravastar with a mass $10^{6} M_{\odot}$, such peak frequencies typically appear within the optimal sensitivity band of LISA.

Another type of $\mathrm{BH}$ no-hair tests is to use the ringdown signal of a $\mathrm{BH}$ e.g. after a merger of two BHs. Ringdown signals or quasi normal modes (QNMs) are exponentially damped sinusoids (see e.g. [280] for a review on QNMs). Since the frequency and the damping time are given by the mass and spin of the ringing $\mathrm{BH}$, a measurement of a single set of complex QNM frequencies allows one to extract the mass and spin, provided that one knows which particular normal mode such a measurement corresponds to. Then, an additional measurement allows one to perform a consistency test of the BH no-hair property [78].

Dreyer et al. [78] studied the prospects of testing the BH no-hair property with LISA by estimating the probability that one fails to identify an actual $\mathrm{BH}$ or one misidentifies non-BH QNMs to be the BH ones. Flanagan and Hughes [77] studied the detectability of these QNMs, while Berti et al. [80,83] and Kamaretsos et al. [86] carried out a Fisher analysis and investigated how accurately one can measure these QNM frequencies with future GW observations. Such Fisher analyses were extended to a Bayesian inference study in [87] by carrying out a parameter estimation study and a model selection analysis between GR and non-GR models. The authors used the $(\ell, m)=(2,2)$ mode to extract the mass and spin of a $\mathrm{BH}$ and used the frequency of the $(\ell, m)=(3,3)$ mode as additional information to check the consistency in the mass-spin plane of a $\mathrm{BH}$, just like the PPK test in binary pulsar observations [3,4,189] and GW tests with parameterized PN waveforms in $[44,45,53]$. They found that ET can measure a $10 \%$ deviation in the frequency of the $(\ell, m)=(2,2)$ mode from GR for a $500 M_{\odot} \mathrm{BH}$ if the luminosity distance is smaller than $\sim 6 \mathrm{Gpc}$ or the redshift smaller than $z \sim 1$. Meidam et al. [89] also carried out a Bayesian model selection study using the TIGER pipeline but for comparatively smaller SNR sources. They found that a $10 \%$ deviation in the frequency of the $(\ell, m)=(2,2)$ mode from GR for a $500 M_{\odot} \mathrm{BH}$ can be detected with $\mathrm{ET}$ if one combines results from $\mathcal{O}(10)$ sources out to $\sim 50 \mathrm{Gpc}(z \leq 5)$. QNMs of boson stars were studied in [74, 75,81,82,88,103], while those of gravastars were calculated in [84, 85, 100].

2.2.5. Systematics In this subsection, we explain possible systematic errors on probing GR with GW observations of BHs. One origin of such systematics is some gas around BHs that forms accretion disks. Narayan [106] studied such an effect on an EMRI embedded in an advection-dominated accretion flow disk. He estimated the amount of the hydrodynamic drag on the small compact object and found that the effect is 
negligible. Barausse and Rezzolla [107] studied the contribution of a non-self-gravitating torus around a SMBH. They found that the effect of the hydrodynamic drag is typically subdominant compared to the radiation reaction, though certain situations exist, where such an effect may be detectable with LISA. Yunes et al. [109] and Kocsis et al. [110] investigated various effects of a geometrically thin, standard model accretion disk on an EMRI in great detail, including the mass accretion, disk's self-energy, hydrodynamic drag, torques from spiral arms, and resonant interactions that are similar to planetary migrations [281, 282]. Among these, they found that the migration gives the dominant contribution to GWs, which may give large impacts on parameter estimation.

Barausse et al. [104, 105] also studied astrophysical systematic errors on testing GR with GWs in detail. They found that such systematics should not be important for eLISA, except for thin disks around EMRIs. However, they argue that EMRIs that can be detected by eLISA will likely to have thick disks instead of thin ones. This is because eLISA can only detect EMRI signals within the redshift $z \sim 0.7$, and galactic nuclei within the local universe are typically quiescent [283,284]. The authors mention that only a few percent of all the EMRIs detected with eLISA may have thin disks. Given that current estimated event rate of EMRIs with eLISA is 5-50 per year, it is likely that matter effects do not significantly affect the tests of GR with EMRIs. They also derived intrinsic lower bounds on non-GR effects to GWs, below which such effects are buried under astrophysical ones. Moreover, they estimated systematic errors on ringdown signals. For example, they found that a small matter distribution with mass $\delta M$ around an EMRI with central BH's mass $M$ can affect the ringdown frequency by roughly $0.05 \%\left[\delta M /\left(10^{-3} M\right)\right]$, which places an intrinsic lower bound on the non-GR effect to be probed with GW ringdowns.

Regarding comparable-mass binaries, Ref. [111] studied the effect of circumbinary disks around such binaries. The orbital angular momentum of a binary is transferred to a circumbinary disk through the tidal/resonant interaction induced by the time-dependent gravitational potential of the binary [285-287], and through the mass accretion of the gas from the inner edge of the circumbinary disk onto the central binary [288]. Irrespective of details of the circumbinary disk model, the authors found that the ratio between the angular momentum transferred from the binary to the disk to the orbital angular momentum of the binary as $C_{1} \dot{M} / \mu$, where $\dot{M}$ is the mass accretion rate, $\mu$ is the reduced mass and $C_{1}$ is a factor of $\mathcal{O}(1)$. For example, the three-dimensional magnetohydrodynamic simulation [289] shows $C_{1} \sim 0.65$. The authors then derived the leading effect of such a circumbinary disk to GWs from the central binary, and found that such an effect enters at $-4 \mathrm{PN}$ order relative to the leading radiation reaction effect. Carrying out a Fisher analysis, they found that the measurement accuracy of the accretion rate $\Delta \dot{M}$ with a $5 y$ r observation of DECIGO at a source distance of $3 \mathrm{Gpc}$ leads to

$$
\frac{\Delta \dot{M}}{\dot{M}_{\mathrm{Edd}}} \approx 1.0 \times 10^{-2} C_{1}^{-1}\left(\frac{m}{10 M_{\odot}}\right)^{10 / 23}
$$

for the total mass $m \lesssim 10^{3} M_{\odot}$, where $\dot{M}_{\text {Edd }}$ is the Eddington accretion rate. However, 
such an event turns out to be rare. When a binary travels through a dense molecular cloud, the gas within the Bondi-Hoyle-Lyttleton radius [290] will form a circumbinary disk. Assuming such a situation, the authors derived the expected number of events with which the accretion rate can be measured with DECIGO as

$$
N_{\text {disk }} \sim 6.0 \times 10^{-2} C_{1}^{-9 / 5}\left(\frac{m}{10 M_{\odot}}\right)^{117 / 115}\left(\frac{v_{\infty}}{20 \mathrm{~km} \mathrm{~s}^{-1}}\right)^{-27 / 5}\left(\frac{n_{\text {gal }}}{0.01 \mathrm{Mpc}^{-3}}\right)
$$

where $v_{\infty}$ and $n_{\text {gal }}$ correspond to the bulk velocity and the number density of galaxies respectively.

Regarding systematics from effects other than accretion disks, Yunes et al. [108] studied the contribution of another SMBH on an EMRI. They found that the acceleration acting on a binary in a time-independent external gravitational field gives again a $-4 \mathrm{PN}$ correction to the waveform phase, and such an effect might be detected with LISA if the perturber is a few tenths of a parsec away from an EMRI. Such a $-4 \mathrm{PN}$ correction degenerates with the correction in RS-II braneworld model (see Sec. 6.2.2) and that in varying $G$ theories [227]. However, notice that astrophysical environmental effects are unique to each source, while coupling constants in non-GR theories are typically universal. Hence, one may be able to distinguish the former from non-GR corrections to GWs by detecting signals from multiple sources. A $-4 \mathrm{PN}$ correction also arises from the time evolution of the redshift (redshift drift) [291-294], though such an effect will not be problematic as long as one has a good cosmological model. Barausse et al. [104,105] also studied systematics due to non-vanishing charges of BHs, cosmological constant, magnetic fields and dark matter, but the effects are negligible. EMRI GWs also suffer from systematics due to mismodeling of the waveform. For example, the self-force contribution is not fully understood $[112,113]$, though such an effect is suppressed by the mass ratio of a binary.

\subsection{Generic Scalar Corrections to GR}

A large portion of the literature on corrections to GR is focused on theories whose field content in the gravity sector consists of the metric and one additional dynamical, long-ranged scalar field. There is a good pragmatic reason for studying theories of this form: they are the simplest possible corrections to GR. Besides convenience, there is also good theoretical motivation to study these theories. In order for an interaction to be "gravitational" in nature, it should be long-ranged and couple weakly to matter. Coupled with the desire to have no preferred frame, these criteria motivate the study of theories with the metric and a scalar.

Unfortunately, there are still an infinite space of theories which are deformations of GR and include a long-ranged scalar field which couples weakly to matter. Rather than studying them one-by-one, here we discuss how to parameterize over theory space.

Stein and Yagi [59] provided one approach to generically classify these theories and their phenomenology. Unlike the other approaches described in this Section, Stein and Yagi (henceforth SY) did not only parameterize observables. Rather, they used a hybrid 
approach where they parameterized the theory, the properties of compact objects, the multipoles of binaries and the scalar radiation they emit; and from these to determine the observables. This approach more closely connects observables to the theoretical corrections that give rise to them.

2.3.1. Parameterization of Theories In Ref. [59], SY considered theories in the Jordan frame, such that matter only couples to the metric in this frame. Thus the scalar field only interacts by coupling non-minimally to curvature, with an action given by (in the conventions of SY),

$$
\begin{array}{rlrl}
S & =S_{\mathrm{EH}}+S_{\mathrm{kin}}+S_{\mathrm{int}}+S_{\mathrm{mat}}, & & \\
S_{\mathrm{EH}} & =\int \frac{1}{2} m_{\mathrm{pl}}^{2} R \sqrt{-g} d^{4} x, & & S_{\mathrm{kin}}=\int-\frac{1}{2}\left(\partial_{a} \vartheta\right)\left(\partial^{a} \vartheta\right) \sqrt{-g} d^{4} x, \\
S_{\mathrm{int}} & =\int \mathcal{L}_{\text {int }}[\vartheta, g, \epsilon, \nabla, R] \sqrt{-g} d^{4} x, & S_{\mathrm{mat}}=S_{\mathrm{mat}}[\Psi, g],
\end{array}
$$

where $m_{\mathrm{pl}}^{2}=(8 \pi G)^{-1}, \nabla$ is the Levi-Civita connection of the metric $g$ and $\epsilon$ its volume form, and $S_{\text {mat }}$ is the matter action, where $\vartheta$ does not appear. Here there is no potential for $\vartheta$, because a nontrivial potential would lead to a short-ranged scalar field.

Of course there is still an infinite functional freedom in $\mathcal{L}_{\text {int }}$. To make progress, SY use the effective field theory (EFT) approach and expand $\mathcal{L}_{\text {int }}$ in a power series in $\vartheta$ and truncate at linear order. This expansion is not capable of expressing theories which rely on strong self-interaction, such as nonlinear Galileons where the self-interaction creates a screening mechanism.

Terms at zeroth order do not source $\vartheta$. At first order in $\vartheta$ it is possible to integrate by parts and remove any derivative from $\vartheta$. Therefore the most general interaction Lagrangian which is homogeneous of degree one in $\vartheta$ may be put into the form of a sum of terms like

$$
\mathcal{L}_{\text {int }} \sim \vartheta T\left[g, \epsilon^{0,1}, \nabla^{d}, R^{r}\right],
$$

where $T[\cdot]$ is some scalar invariant built from the arguments. Each term is constructed from $d$ derivatives, $r$ curvature tensors, and $|\epsilon|=0$ or $|\epsilon|=1$ copies of the $\epsilon$ tensor (no more copies may appear due to an identity). This requires introducing a new length scale, $\ell$, for dimensional correctness; SY parameterize this as

$$
\mathcal{L}_{\text {int }} \sim\left(m_{\mathrm{pl}} \ell\right) \ell^{\wp} \vartheta T\left[g, \epsilon^{0,1}, \nabla^{d}, R^{r}\right],
$$

where for dimensional correctness we have $\wp=d+2 r-3$. The new length scale $\ell$ also controls the strength of this interaction. This part of theory space is parameterized by the integers $(|\epsilon|, d, r)$ and the length $\ell$. This parameterization includes other theories we discuss in this review, including some scalar-tensor theories (Sec. 3) and quadratic gravity theories (Sec. 5); see Table 1 for the parameters of these theories. 


\begin{tabular}{rccccccccc}
\hline Theory & $|\epsilon|$ & $\mathrm{d}$ & $\mathrm{r}$ & $\wp^{a}$ & $\ell_{\mathrm{BH}}$ & $\ell_{\mathrm{NS}}$ & $\ell_{\mathrm{rad}}^{\mathrm{HH}}$ & $\ell_{\mathrm{rad}}^{\mathrm{HS}}$ & $\ell_{\mathrm{rad}}^{\mathrm{SS}}$ \\
\hline "Scalar-tensor" & 0 & 0 & 1 & -1 & $-^{b}$ & 0 & $-^{b}$ & 1 & 1 \\
EDGB & 0 & 0 & 2 & 1 & 0 & $2^{c}$ & 1 & 1 & $3^{c}$ \\
$\mathrm{dCS}$ & 1 & 0 & 2 & 1 & 1 & 1 & 2 & 2 & 2 \\
\hline
\end{tabular}

Table 1. Parameters of three example theories with a long-ranged, weakly-coupling gravitational scalar. The parameters of the Lagrangian are $(|\epsilon|, d, r)$, and the multipole parameters are $\left(\ell_{\mathrm{BH}}, \ell_{\mathrm{NS}}, \ell_{\mathrm{rad}}\right)$. The combinations $\mathrm{HH} / \mathrm{HS} / \mathrm{SS}$ are the three binary combinations of $\mathrm{BH}(\mathrm{H})$ and $\mathrm{NS}(\mathrm{S})$. In this Table we have $\ell_{\mathrm{rad}}=1+\min \left(\ell_{1}, \ell_{2}\right)$. Notes: (a) Not independent, $\wp=2 r+d-3$. (b) BHs have no hair in classical scalar-tensor theories. (c) This is expected but has not yet been calculated. Table from Ref. [59]

2.3.2. Parameterization of Compact Object Properties Determining the leading effect of the scalar interaction on the orbital dynamics of a binary system requires knowledge of the scalar field profile sourced by each of the members of the binary system. This profile is sometimes called the "scalar hair" of an object. The structure of this hair must be determined through a strong-field matching calculation in each theory of interest. However, again in the interest of creating a parameterized framework, SY simply parameterize this hair by the lowest non-vanishing multipole number $\ell_{\text {body }}$ (an integer) which comes from this matching calculation. It is the lowest non-vanishing moments of the two bodies which control the scalar-mediated interaction between the two bodies. In several theories (e.g. Brans-Dicke and EdGB), BHs and NSs have different leading scalar multipole numbers. Therefore the body moments need to be parameterized through both $\ell_{\mathrm{NS}}$ and $\ell_{\mathrm{BH}}$. See Table 1 for the parameters in a number of theories.

For a given set of theory parameters $(|\epsilon|, d, r)$ and scalar multipole number $\ell_{\text {body }}$, it is possible to use curvature and compactness scalings to determine the scaling law of the scalar multipole moment $\mu^{Q}$ with $Q=q_{1} q_{2} \cdots q_{\ell_{\text {body }}}$ a multi-index of valence $|Q|=q=\ell_{\text {body }}$. This scaling is determined up to some dimensionless integrals of order unity. These symmetric tracefree (STF) tensors $\mu^{Q}$ are essentially Wilson coefficients for the world-line effective action.

For parity-even theories $(|\epsilon|=0)$, SY determine the scaling of $\mu^{Q}$ as

$$
\mu^{Q} \sim\left(m_{\mathrm{pl}} \ell\right)\left(\frac{\ell}{R_{*}}\right)^{\wp} C_{*}^{r} R_{*}^{q}
$$

where $R_{*}$ is the radius of the body, and $C_{*}=G M_{*} / R_{*}$ is the compactness of the body, where $M_{*}$ is the mass of the body. The scaling estimate of Eq. (40) agrees with the strong-field matching calculation in EdGB [176]. For parity-odd theories $(|\epsilon|=1)$, SY determine the scaling as

$$
\mu^{Q} \sim\left(m_{\mathrm{pl}} \ell\right) v_{\mathrm{eq}} \hat{S}\left(\frac{\ell}{R_{*}}\right)^{\wp} C_{*}^{r} R_{*}^{q}=\left(m_{\mathrm{pl}} \ell\right) S\left(\frac{\ell}{R_{*}}\right)^{\wp} C_{*}^{r-1} R_{*}^{q-2}
$$

where $S^{i}$ is the spin vector of the body (with dimensions of length squared), $\hat{S}^{i}$ the unit normal vector in the direction of $S^{i}$, and $v_{\text {eq }}$ the velocity at the equator of the rotating 
body. The scaling estimate of Eq. (41) agrees with the strong-field matching calculation in dCS [259, 295, 296].

The scalings presented in Eq. (40) and (41) may not capture effects such as spontaneous, dynamical, or induced scalarization in scalar-tensor theories. However, the fact still remains that such scalar multipole moments do exist, but in these cases they must be promoted to be environment-dependent. Therefore below we will present formulas with the $\mu^{Q}$ 's left general, and also substitute in the scalings of (40) and (41).

2.3.3. Binary, Scalar Interaction, and Pulsar Timing Bounds Let us now consider a binary system of compact objects, either two BHs (HH), two NSs (SS), or one of each (HS). The presence of a dynamical scalar and scalar "hairs" induces a new interaction between the two bodies besides the interaction through the metric (the Newtonian and post-Newtonian interactions).

In some theory where the scalar hairs of a $\mathrm{BH}$ and NS may differ $\left(\ell_{\mathrm{BH}} \neq \ell_{\mathrm{NS}}\right)$, each of these three cases may experience a different scalar pole-pole interaction. The electromagnetic analogy is that the electric monopole-monopole, monopole-dipole, dipolequadrupole, etc. interactions all have different radial and angular dependences. Therefore to continue to be generic, let the bodies with masses $m_{1,2}$ have scalar multipole moment tensors $\mu_{1}^{S}$ and $\mu_{2}^{T}$, with $|S|=s$ the scalar multipole number of body 1 and respectively $|T|=t$ for body 2 .

Through the EFT technique of integrating out the scalar field, SY show that the presence of these scalar hairs induces an effective world-line interaction given by*

$$
\begin{aligned}
L_{\times}\left[\mathbf{x}_{1}, \mathbf{x}_{2}\right] & =(-)^{s} 4 \pi \mu_{1}^{S} \mu_{2}^{T}\left(\partial_{S T} \frac{1}{r_{2}}\right)\left[\mathbf{x}_{1}\right] \\
& =(-)^{t} 4 \pi \mu_{1}^{S} \mu_{2}^{T}\left(\partial_{S T} \frac{1}{r_{1}}\right)\left[\mathbf{x}_{2}\right], \\
L_{\times}\left[\mathbf{x}_{1}, \mathbf{x}_{2}\right] & =(-)^{t} 4 \pi(2 s+2 t-1) ! ! \frac{\mu_{1}^{S} \mu_{2}^{T} n_{12}^{\langle S T\rangle}}{r_{12}^{1+s+t}} .
\end{aligned}
$$

Here $r_{A} \equiv\left|\mathbf{x}-\mathbf{x}_{A}\right|$ is the distance from some field point $\mathbf{x}$ to body $A, \mathbf{x}_{12} \equiv \mathbf{x}_{1}-\mathbf{x}_{2}$ is the vector which points from body 2 to body $1, r_{12} \equiv\left|\mathbf{x}_{12}\right|$ is the distance between the two bodies, $\mathbf{n}_{12} \equiv \mathbf{x}_{12} / r_{12}$ is the unit normal vector which points from body 2 to body 1 , and $\langle\cdots\rangle$ is the STF operation on the enclosed indices. Here we see that the sum $s+t$ controls the radial and angular dependence of the interaction. For $s=0=t$ we have the same structure as the Kepler interaction, which simply renormalizes $G \rightarrow \mathcal{G}_{A B}$ pairwise for each combination of bodies $A, B$. This is similar to the Nordtvedt effect, and the conservative effect can not be detected in an isolated binary. However, all other choices of $s, t$ lead to post-Newtonian corrections.

This effective interaction Lagrangian leads to a conservative shift in the binding

* This corrects a sign error in [59] and a number of other articles which made the same mistake in the integrating-out procedure. This sign error propagates through to the force, pericenter precession, etc. 
energy, which is simply $\delta E_{\text {bind }}=-L_{\times}\left[\mathbf{x}_{1}, \mathbf{x}_{2}\right]$. It also leads to an additional scalarmediated force between the two bodies, which SY find to be

$$
F_{1}^{i}=(-)^{t+1} 4 \pi(2 s+2 t+1) ! ! \frac{\mu_{1}^{S} \mu_{2}^{T} n_{12}^{\langle i S T\rangle}}{r_{12}^{2+s+t}},
$$

and similarly for the force on body 2 due to 1 , which is easily seen to be $F_{2}^{i}=-F_{1}^{i}$.

From this additional force it is possible to find the conservative correction to the motion of the binary. The most useful observable is the correction to the pericenter precession. Stein and Yagi compute this via Gauss perturbation of the orbital elements. The net result of the calculation is that the excess pericenter precession is given by

$$
\langle\delta \dot{\omega}\rangle=\frac{1}{T} \frac{p^{2}}{G m} \mathcal{A} \mu_{1}^{S} \mu_{2}^{T}\left[-\frac{1}{e} \frac{s+t+1}{2 s+2 t+1} I_{1}^{S T}+\frac{1}{e} \hat{L}^{i} I_{2}^{i S T}-\cot \iota \frac{s+t+1}{2 s+2 t+3} \epsilon_{i j k} \hat{L}^{j} I_{3}^{i k S T}\right]
$$

where $p$ is the semi-latus rectum of the background (Keplerian) orbit, $e$ its eccentricity, $T$ its period, $\iota$ the inclination (relative to the line of sight), $\hat{L}^{i}$ the unit vector in the direction of the angular momentum, $I_{1,2,3}$ are three tensor-valued dimensionless integrals of order unity which depend on the eccentricity, and defining the parameter

$$
\mathcal{A} \equiv \frac{1}{\mu}(-)^{t+1} 4 \pi(2 s+2 t+1) ! ! p^{-(2+s+t)},
$$

where $\mu \equiv m_{1} m_{2} / m$ is the reduced mass, and $m=m_{1}+m_{2}$ is the total mass of the binary. Comparing this with the leading GR pericenter precession, we see that this is a relative $+(s+t-1) \mathrm{pN}$ order correction. Since $s$ and $t$ should have the same parity, we see that this is always an odd relative $\mathrm{pN}$ order correction.

By using the scaling laws from Eqs. (40) and (41) it is possible to derive the scaling of the pericenter precession in terms of the size and compactness of the bodies in the binary. For parity even $(|\epsilon|=0)$ theories, SY find this to be

$$
\begin{aligned}
\frac{\langle\delta \dot{\omega}\rangle}{\langle\dot{\omega}\rangle_{\mathrm{GR}}} \sim & (-)^{t+1}(2 s+2 t+1) ! ! \frac{\ell^{2}}{(G m)(G \mu)}\left(\frac{\ell^{2}}{R_{1} R_{2}}\right)^{\wp}\left(C_{1} C_{2}\right)^{r} \\
& \times\left(\frac{R_{1}}{G m}\right)^{s}\left(\frac{R_{2}}{G m}\right)^{t} f(e) v^{2(s+t-1)}
\end{aligned}
$$

with $v=\left|v_{12}\right|$ the orbital velocity, and $f(e)$ is an $\mathcal{O}(1)$ function that depends on eccentricity. For parity odd $(|\epsilon|=1)$ theories, factors of $v_{\text {eq }}$ for each body must also be included. This result reproduces the known result from dCS [296].

From this calculation it is possible to estimate bounds which can be placed on the dimensional coupling parameter, $\ell$, in some theory. If a binary pulsar system is observed with sufficient precision to measure $\langle\dot{\omega}\rangle$ and two other post-Keplerian parameters, this constitutes a test of GR [3,4]. Let us presume that the variance on the measurement of $\langle\dot{\omega}\rangle$ is $\sigma$. If the measurements are consistent with GR, then we must have that the 


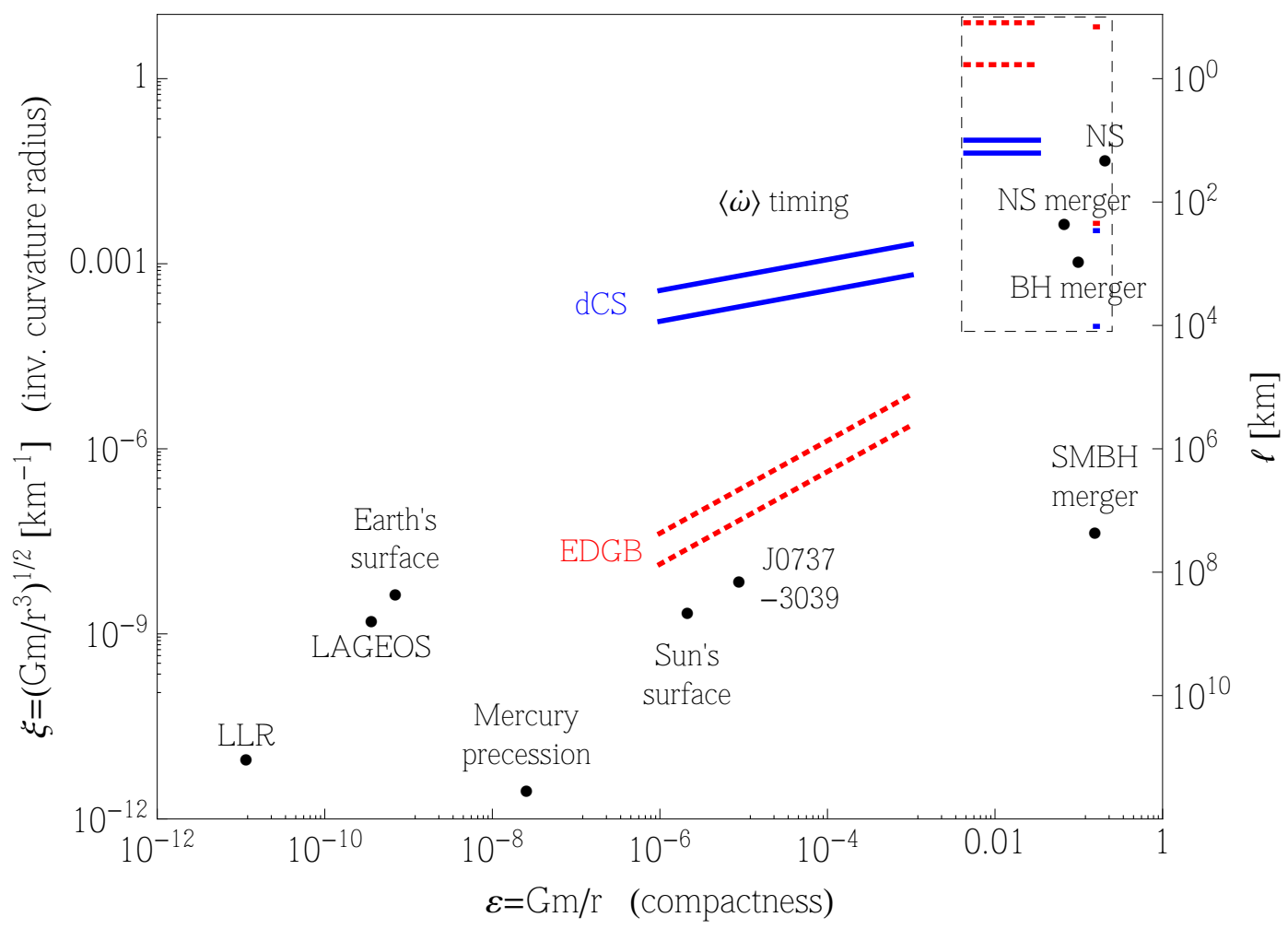

Figure 2. Estimated bounds on $\ell$, as shown on the right vertical axis. Tilted lines present estimates from pericenter precession in Eq. (49), while horizontal lines correspond to those from GWs. The horizontal axis represents the compactness of a binary given by $(\mathrm{Gm} / \mathrm{a})$. Solid (blue) curves are bounds for dCS, while dotted (red) curves are for EdGB. The lower (upper) curve for each theory is the estimate for $|\sigma /\langle\dot{\omega}\rangle| \sim 1\left(|\sigma /\langle\dot{\omega}\rangle| \sim 10^{-2}\right)$. The dashed region is expanded in Fig. 3. Figure from [59].

excess precession [from Eq. (48)] $\langle\delta \dot{\omega}\rangle \lesssim \sigma$ is smaller than this variance, which leads to the bound on $\ell$,

$$
\ell^{2+2 \wp} \lesssim \frac{|\sigma|}{\langle\dot{\omega}\rangle} \frac{G m G \mu R_{1}^{\wp} R_{2}^{\wp}}{(4 s+1) ! ! C_{1}^{r} C_{2}^{r}}\left[\frac{(G m)^{2}}{R_{1} R_{2}}\right]^{s} v^{2(1-2 s)} .
$$

Here we have set $t=s$ since all known binary systems are two NSs, and therefore they will have the same type of scalar hair. These scaling estimates of bounds are presented in Fig. 2.

2.3.4. Parameterization of Scalar Radiation A binary system where one or both bodies sources scalar hair will emit scalar radiation. This in turn will affect the rate of inspiral of the binary and thus change the gravitational waveform. The scalar radiation may be found in terms of derivatives of the binary's combined scalar moments [297],

$$
\theta=\sum_{q=0}^{\infty} \frac{(-)^{q}}{q !}\left(\frac{1}{r} \mu_{\mathrm{bin}}^{Q}\right)_{, Q}
$$


where $r$ is the distance to the binary, and where

$$
\mu_{\mathrm{bin}}^{Q}(u)=\int_{\mathcal{M}} \tau_{\text {eff }}(u, \mathbf{x}) x^{Q} d^{3} x
$$

with the point-particle effective source is given by the superposition

$$
\tau_{\text {eff }}=(-)^{s} \mu_{1}^{S} \partial_{S} \delta^{(3)}\left(\mathbf{x}-\mathbf{x}_{1}\right)+(-)^{t} \mu_{2}^{T} \partial_{T} \delta^{(3)}\left(\mathbf{x}-\mathbf{x}_{2}\right) .
$$

Stein and Yagi find the $q$ th binary moment as

$$
\mu_{\text {bin }}^{Q}= \begin{cases}\frac{q !}{s !} \mu_{1}^{\left(k_{1} \cdots k_{s}\right.} x_{1}^{\left.k_{s+1} \cdots k_{q}\right)}+(1 \leftrightarrow 2) & q \geq s \\ 0 & \text { otherwise. }\end{cases}
$$

Now for each $q$ in Eq. (50) one must compute $\left(\mu_{\text {bin }}^{Q} / r\right)_{, Q}$. It is not immediately clear which radiative binary moment will dominate this sum. Therefore SY simply parameterized this as $\ell_{\text {rad }}$, so that the radiative solution may be approximated as

$$
\theta_{\mathrm{rad}} \approx \frac{1}{r} \frac{n^{W}}{w !}(w) \mu_{\mathrm{bin}}^{W}(u)
$$

where $w=|W|=\ell_{\text {rad }},{ }^{(q)} f=(\partial / \partial t)^{q} f$, and $n^{i}$ is the radial outward unit vector at some field point, when the binary is taken at the origin of coordinates.

We know that $\ell_{\text {rad }}$ must be greater than or equal to $\min \left(\ell_{1}, \ell_{2}\right)$, the smaller of the two individual bodies' scalar multipole moments (since all binary moments of lower valence will vanish). It is possible that the dominant radiative multipole moment is simply $\ell_{\text {rad }}=\min \left(\ell_{1}, \ell_{2}\right)$. However, the computation of ${ }^{(w)} \mu_{\text {bin }}^{W}$ for $w=\min \left(\ell_{1}, \ell_{2}\right)$ does not contain any time derivatives of the separation vector between the two bodies. The $w$ th derivative of this quantity depends on changes to the internal structure of the bodies, or on the precession of their spins.

On the other hand, for $w=1+\min \left(\ell_{1}, \ell_{2}\right),{ }^{(w)} \mu_{\text {bin }}^{W}$ will indeed involve a time derivative of the separation vector, will thus vary on an orbital timescale, and is thus expected to be important. The binary moment for this $w$ is

$$
\mu_{\mathrm{bin}}^{a S}=w x_{1}^{(a} \mu_{1}^{S)}+(1 \leftrightarrow 2) .
$$

This sum may contain only one term if e.g. $\ell_{1} \neq \ell_{2}$ if the binary is a BH-NS system. For a circular orbit, SY find the relevant time derivative to be

$$
{ }^{(w)} \mu_{\mathrm{bin}}^{a S}=\frac{w}{(G m)^{s}}\left\{\begin{array}{lll}
(-)^{(1+s) / 2} & \mu_{\mathrm{red}}^{(S} n_{12}^{a)} v^{3 s+1}, & |\epsilon|=1 \\
(-)^{s / 2} & \mu_{\mathrm{red}}^{(S} v_{12}^{a)} v^{3 s}, & |\epsilon|=0
\end{array}\right.
$$

where $\ell_{\text {rad }}=w=1+s=1+\min \left(\ell_{1}, \ell_{2}\right)$, and where SY have defined a certain combination

$$
\mu_{\mathrm{red}}^{S} \equiv\left[\frac{m_{2}}{m} \mu_{1}^{S}-\frac{m_{1}}{m} \mu_{2}^{S}\right]
$$


By combining this calculation with the estimates of Eqs. (40) and (41), it is possible to estimate the scaling of $\mu_{\text {bin }}^{W}$ and its time derivatives. This scaling is controlled by the difference $s-\wp$. Stein and Yagi present this scaling estimate for the four cases $s-\wp=-1,0,+1,+2$.

This quantity appears in the energy flux due to scalar radiation, which SY find to be

$$
\dot{E}^{(\vartheta)}=-\frac{1}{(w !)^{2}} \frac{4 \pi}{2 w+1} \delta_{(V W)}\left\langle{ }^{(w+1)} \mu_{\mathrm{bin}}^{V}{ }^{(w+1)} \mu_{\mathrm{bin}}^{W}\right\rangle
$$

where $|W|=|V|=w$. Using the above assumptions, this result becomes

$$
\dot{E}^{(\vartheta)}=-\frac{1}{(s !)^{2}} \frac{4 \pi}{2 w+1} \frac{1}{(G m)^{2 w}} \mu_{\mathrm{red}}^{S} \mu_{\mathrm{red}}^{T} \delta_{(a b S T)} \begin{cases}\left\langle n_{12}^{a} n_{12}^{b} v^{6 s+8}\right\rangle, & |\epsilon|=0 \\ \left\langle v_{12}^{a} v_{12}^{b} v^{6 s+6}\right\rangle, & |\epsilon|=1\end{cases}
$$

where $|S|=|T|$ and $w=1+s$.

This is easily seen to be of $+(3 s-1) \mathrm{pN}$ order relative to the leading GR energy flux due to quadrupole radiation:

$$
\frac{\dot{E}^{(\theta)}}{\dot{E}^{\mathrm{GW}}} \sim \frac{5 \pi^{2}}{(2 w+1)(s !)^{2}} \frac{m_{\mathrm{pl}}^{2}}{\mu^{2}} \frac{\left|\mu_{\mathrm{red}}^{S}\right|^{2}}{(G m)^{2 s}} v^{6 s-2} .
$$

Using the scaling estimates, which recall are controlled by $s-\wp$, SY derive that for $|\epsilon|=0$ theories:

$$
\begin{array}{lrl}
\frac{\dot{E}^{(\vartheta)}}{\dot{E}^{\mathrm{GW}}} \sim \frac{\eta^{-4} v^{6 s-2}}{(2 w+1)(s !)^{2}}\left(\frac{\ell}{G m}\right)^{2+2 \wp} \frac{\delta m^{2}}{m^{2}} & (s-\wp=-1) \\
\frac{\dot{E}^{(\vartheta)}}{\dot{E}^{\mathrm{GW}}} \sim \frac{\eta^{-2} v^{6 s-2}}{(2 w+1)(s !)^{2}}\left(\frac{\ell}{G m}\right)^{2+2 \wp} \frac{\delta m^{2}}{m^{2}} & (s-\wp=0) \\
\frac{\dot{E}^{(\vartheta)}}{\dot{E}^{\mathrm{GW}}} \sim \frac{v^{6 s-2}}{(2 w+1)(s !)^{2}}\left(\frac{\ell}{G m}\right)^{2+2 \wp} & (s-\wp=+1) \\
\frac{\dot{E}^{(\vartheta)}}{\dot{E}^{\mathrm{GW}}} \sim \frac{v^{6 s-2}}{(2 w+1)(s !)^{2}}\left(\frac{\ell}{G m}\right)^{2+2 \wp} \frac{\delta m^{2}}{m^{2}}, &
\end{array}
$$

where $\eta=m_{1} m_{2} / m^{2}=\mu / m$ is the symmetric mass ratio. These scaling estimates agree with previously-derived results in specific theories such as EdGB (where $s-\wp=-1$ for two BHS) and dCS (where $s-\wp=0$ for two BHs; though note this theory has $|\epsilon|=1)[38]$.

2.3.5. Connection to PPE and Estimates of $G W$ Bounds There are four physical effects which SY identify that correct the inspiral and thus the GW signal. These effects are:

(i) The conservative scalar pole-pole interaction, which modifies the binding energy, and the relation between $r_{12}$ and the orbital frequency $\omega$ (the "Kepler" relation). This was discussed in Sec. 2.3.3. 
(ii) A conservative effect from the correction to the metric multipole moments of the bodies. For example, a shift in the bodies' metric quadrupoles from the GR values will shift the metric monopole-quadrupole interaction energy from the GR value.

(iii) The dissipative effect of the energy lost due to scalar radiation.

(iv) A dissipative correction to how much energy is radiated in GWs.

Effects (i) and (iii) were discussed above in Secs. 2.3.3 and 2.3.4. Each effect may contribute to the gravitational waveform, though they may all enter at different postNewtonian orders; therefore we do not know, a priori, which effect will dominate the correction to the GR waveform. In EdGB, for a BH-BH system, effect (iii) is a relative $-1 \mathrm{PN}$ effect and thus dominates [38]. Meanwhile, in dCS, all of these corrections enter the waveform at the same order: $+2 \mathrm{pN}$ relative to the leading GR phase [39, 296].

Since Ref. [59] focused only on the scalar interaction, they choose to simply parameterize effects (ii) and (iv) for the purposes of computing waveforms and mapping to the PPE framework explained in Sec. 2.2.3.

To parameterize effect (ii), SY wrote the shift to the binding energy due to the metric deformation as

$$
\delta E_{\mathrm{bind}}^{\mathrm{def}}=\frac{C_{\mathrm{def}}}{r^{1+n_{\mathrm{def}}}} .
$$

If this is the leading conservative non-GR correction, then $C_{\mathrm{def}}=E_{\mathrm{GR}} A_{\mathrm{ppE}}$ and $1+n_{\mathrm{def}}=p_{\mathrm{ppE}}$ [54]. If there is another effect at this same post-Newtonian order, then $C_{\text {def }}$ adds linearly to $E_{\mathrm{GR}} A_{\mathrm{ppE}}$. Otherwise, this contribution is sub-dominant.

To parameterize effect (iv), SY write the correction in the energy flux due to the correction in GWs as

$$
\dot{E}^{(h)}=C_{h}\left(\frac{G m}{r_{12}}\right)^{5+n_{h}} .
$$

Again if this is the leading dissipative non-GR correction, then this corresponds to $C_{h}=\dot{E}_{\mathrm{GR}} B_{\mathrm{ppE}}$ and $5+n_{h}=q_{\mathrm{ppE}}$ [54]. If there is another effect at the same order, then $C_{h}$ adds linearly to $\dot{E}_{\mathrm{GR}} B_{\mathrm{ppE}}$. Otherwise, this contribution is sub-dominant.

Having all effects (i-iv) parameterized, SY are then able to go through the standard post-Newtonian procedure to find the corrections to the GW phase. From the two conservative effects, they find the combined binding energy in frequency space is

$$
E_{\text {bind }} \sim \mu(G m \omega)^{2 / 3}\left[1+\frac{1}{G m \mu} \frac{\left|\mu_{1} \mu_{2}\right|}{(G m)^{2 s}}(G m \omega)^{4 s / 3}+\frac{1}{G m \mu} \frac{C_{\text {def }}}{(G m)^{n_{\text {def }}}}(G m \omega)^{2 n_{\mathrm{def}} / 3}\right] .
$$

Including these corrections and the two dissipative effects, they then find the corrected energy flux in frequency space as

$$
\begin{aligned}
\dot{E} \sim \frac{\eta^{2}}{G}(G m \omega)^{10 / 3}[1 & +\frac{1}{G m \mu} \frac{\left|\mu_{1} \mu_{2}\right|}{(G m)^{2 s}}(G m \omega)^{4 s / 3}+\frac{1}{G m \mu} \frac{C_{\mathrm{def}}}{(G m)^{n_{\mathrm{def}}}}(G m \omega)^{2 n_{\mathrm{def}} / 3} \\
& \left.+\frac{G}{\eta^{2}(G m)^{2}} \frac{\left|\mu_{\mathrm{red}}\right|^{2}}{(G m)^{2 s}}(G m \omega)^{2 s-2 / 3}+\frac{G C_{h}}{\eta^{2}}(G m \omega)^{2 n_{h} / 3}\right] .
\end{aligned}
$$


By going through the stationary phase approximation, SY can then find the correction to the gravitational waveform phase due to these 4 effects. They find

$$
\begin{aligned}
\Psi(f) \sim \frac{1}{\eta}(\pi G m f)^{-5 / 3}+\frac{1}{\eta^{2} G m^{2}} \frac{\left|\mu_{1} \mu_{2}\right|}{(G m)^{2 s}}(\pi G m f)^{(4 s-5) / 3} \\
+\frac{1}{\eta^{2} G m^{2}} \frac{C_{\mathrm{def}}}{(G m)^{n_{\mathrm{def}}}}(\pi G m f)^{\left(2 n_{\mathrm{def}}-5\right) / 3} \\
+\frac{1}{\eta^{3} G m^{2}} \frac{\left|\mu_{\mathrm{red}}\right|^{2}}{(G m)^{2 s}}(\pi G m f)^{(6 s-7) / 3}+\frac{G C_{h}}{\eta^{3}}(\pi G m f)^{\left(2 n_{h}-5\right) / 3}
\end{aligned}
$$

From this equation it is straightforward to read off the PPE coefficients in Eq. (32). The corresponding $b_{\mathrm{ppE}}$ parameters are

$$
\begin{aligned}
b_{\mathrm{ppE}}^{(i)} & =(4 s-5) / 3 \\
b_{\mathrm{ppE}}^{(i i)} & =\left(2 n_{\mathrm{def}}-5\right) / 3 \\
b_{\mathrm{ppE}}^{(i i i)} & =(6 s-7) / 3 \\
b_{\mathrm{ppE}}^{(i v)} & =\left(2 n_{h}-5\right) / 3 .
\end{aligned}
$$

The $\beta_{\mathrm{ppE}}$ coefficients for effects (ii) and (iv) are given as

$$
\begin{aligned}
& \beta_{\mathrm{ppE}}^{(i i)} \sim \frac{1}{\eta^{1+2 n_{\mathrm{def}} / 5} G m^{2}} \frac{C_{\mathrm{def}}}{(G m)^{n_{\mathrm{def}}}} \\
& \beta_{\mathrm{ppE}}^{(i v)} \sim \frac{G C_{h}}{\eta^{2+2 n_{h} / 5}} .
\end{aligned}
$$

For effect (i), in the case with $s=t$ and $|\epsilon|=0$, SY find

$$
\beta_{\mathrm{ppE}}^{(i)} \sim \frac{\ell^{2}}{\eta^{1+4 s / 5}(G m)^{2}}\left(\frac{\ell^{2}}{R_{1} R_{2}}\right)^{\wp}\left(\frac{R_{1} R_{2}}{(G m)^{2}}\right)^{s}\left(C_{1} C_{2}\right)^{r} .
$$

Finally, as seen in Eq. (61), different values of $s-\wp$ give different expressions for effect (iii). For $s-\wp=-1,0,+1,+2$, SY find

$$
\begin{array}{llrl}
\beta_{\mathrm{ppE}}^{(i i i)} & \sim \frac{1}{\eta^{(12+6 \wp) / 5}}\left(\frac{\ell}{G m}\right)^{2+2 \wp}\left(\frac{\delta m}{m}\right)^{2} & (s-\wp=-1) \\
\beta_{\mathrm{ppE}}^{(i i i)} \sim \frac{1}{\eta^{(8+6 \wp) / 5}}\left(\frac{\ell}{G m}\right)^{2+2 \wp}\left(\frac{\delta m}{m}\right)^{2} & (s-\wp=0) \\
\beta_{\mathrm{ppE}}^{(i i i)} \sim \frac{1}{\eta^{(4+6 \wp) / 5}}\left(\frac{\ell}{G m}\right)^{2+2 \wp} & (s-\wp=+1) \\
\beta_{\mathrm{ppE}}^{(i i i)} \sim \frac{1}{\eta^{(10+6 \wp) / 5}}\left(\frac{\ell}{G m}\right)^{2+2 \wp}\left(\frac{\delta m}{m}\right)^{2} . & (s-\wp=+2)
\end{array}
$$

This completes the mapping onto the PPE framework. These results agree with previous calculations within specific theories such as EdGB [38] and dCS [38,39].

Having mapped the effects of generic scalar interactions onto the PPE framework, it is possible to reuse PPE parameter estimation studies to determine how $\ell$ can be 
bounded in these theories. Consider a GW inspiral measurement with a given SNR, over a certain frequency range $f_{\min } \leq f \leq f_{\max }$, which is found to be consistent with the predictions of GR. For such a measurement, Cornish et al. [298] established the estimated bound

$$
|\beta| \lesssim \frac{3}{\operatorname{SNR} \Delta u^{b}}
$$

where $\Delta u^{b} \equiv\left|\left(u_{\min }\right)^{b_{\mathrm{ppE}}}-\left(u_{\max }\right)^{b_{\mathrm{ppE}}}\right|$, with the dimensionless frequency parameter $u=\pi G \mathcal{M} f=\eta^{3 / 5} v^{3}$ (recall that $\mathcal{M}=m \eta^{3 / 5}$ is the chirp mass of the inspiral). This can be immediately converted into an estimated bound on $\ell$.

Specifically, for effect (i), SY find the estimated bound

$$
\ell^{2+2 \wp} \lesssim \frac{3}{\operatorname{SNR} \Delta u^{b^{(i)}}} \frac{\eta^{+1+4 s / 5}}{C_{1}^{r} C_{2}^{r}}(G m)^{2} R_{1}^{\wp} R_{2}^{\wp}\left[\frac{(G m)^{2}}{R_{1} R_{2}}\right]^{s} .
$$

Similarly, SY derive inequalities for effect (iii), which are controlled by the difference $s-\wp$. For the four values previously considered, we have

$$
\begin{aligned}
& \ell^{2+2 \wp} \lesssim \frac{3 \eta^{(12+6 \wp) / 5}}{\operatorname{SNR} \Delta u^{b^{(i i i)}}}(G m)^{2+2 \wp}\left(\frac{m}{\delta m}\right)^{2} \quad(s-\wp=-1) \\
& \ell^{2+2 \wp} \lesssim \frac{3 \eta^{(8+6 \wp) / 5}}{\operatorname{SNR} \Delta u^{b^{(i i i)}}}(G m)^{2+2 \wp}\left(\frac{m}{\delta m}\right)^{2} \quad(s-\wp=0) \\
& \ell^{2+2 \wp} \lesssim \frac{3 \eta^{(4+6 \wp) / 5}}{\operatorname{SNR} \Delta u^{b^{(i i i)}}}(G m)^{2+2 \wp} \quad(s-\wp=+1) \\
& \ell^{2+2 \wp} \lesssim \frac{3 \eta^{(10+6 \wp) / 5}}{\operatorname{SNR} \Delta u^{b^{(i i i)}}}(G m)^{2+2 \wp}\left(\frac{m}{\delta m}\right)^{2} . \quad(s-\wp=+2)
\end{aligned}
$$

Some estimated bounds based on these inequalities have been plotted in Fig. 3. Stein and Yagi plot examples arising from BH-BH inspirals in both dCS and EdGB. For each example in each theory, SY plot both bounds arising from effects (i) and (iii) [respectively Eq. (70) and Eqs. (71)]. For each theory, the two example systems considered are a $10 M_{\odot}-11 M_{\odot}$ binary detected in LIGO over the frequency range $20-400 \mathrm{~Hz}$; and a $10 M_{\odot}-10^{6} M_{\odot}$ binary detected in LISA over a frequency range corresponding to 1 year of measurement terminating at a plunge. Both of these examples are assumed to be measured at an SNR of 30, so that Eq. (69) gives $|\beta| \lesssim 0.1 / \Delta u^{b}$.

Qualitatively, we can see in Fig. 3 that stellar mass BHs yield better estimated bounds on $\ell$. This makes sense for theories like dCS and EdGB, which are highercurvature corrections to GR. The curvature at the horizon of a BH goes as $\sim 1 / M^{2}$, so lighter BHs are better probes of these higher-curvature theories.

This analysis used dCS and EdGB as examples, in order to validate the approach against well-studied theories. The utility of this work is that it can easily be reused for a broad class of theories which include a long-ranged scalar field which is non-minimally coupled to curvature. Rather than redoing a whole suite of calculations in every specific theory, one performs a matching calculation for compact objects, and can then plug in parameters $\left(|\epsilon|, d, r, \ell_{\mathrm{BH}}, \ell_{\mathrm{NS}}, \mu_{\mathrm{BH}}^{S}, \mu_{\mathrm{NS}}^{S}\right)$ to immediately find how well such a theory could be constrained by pulsar timing or GW measurements. 


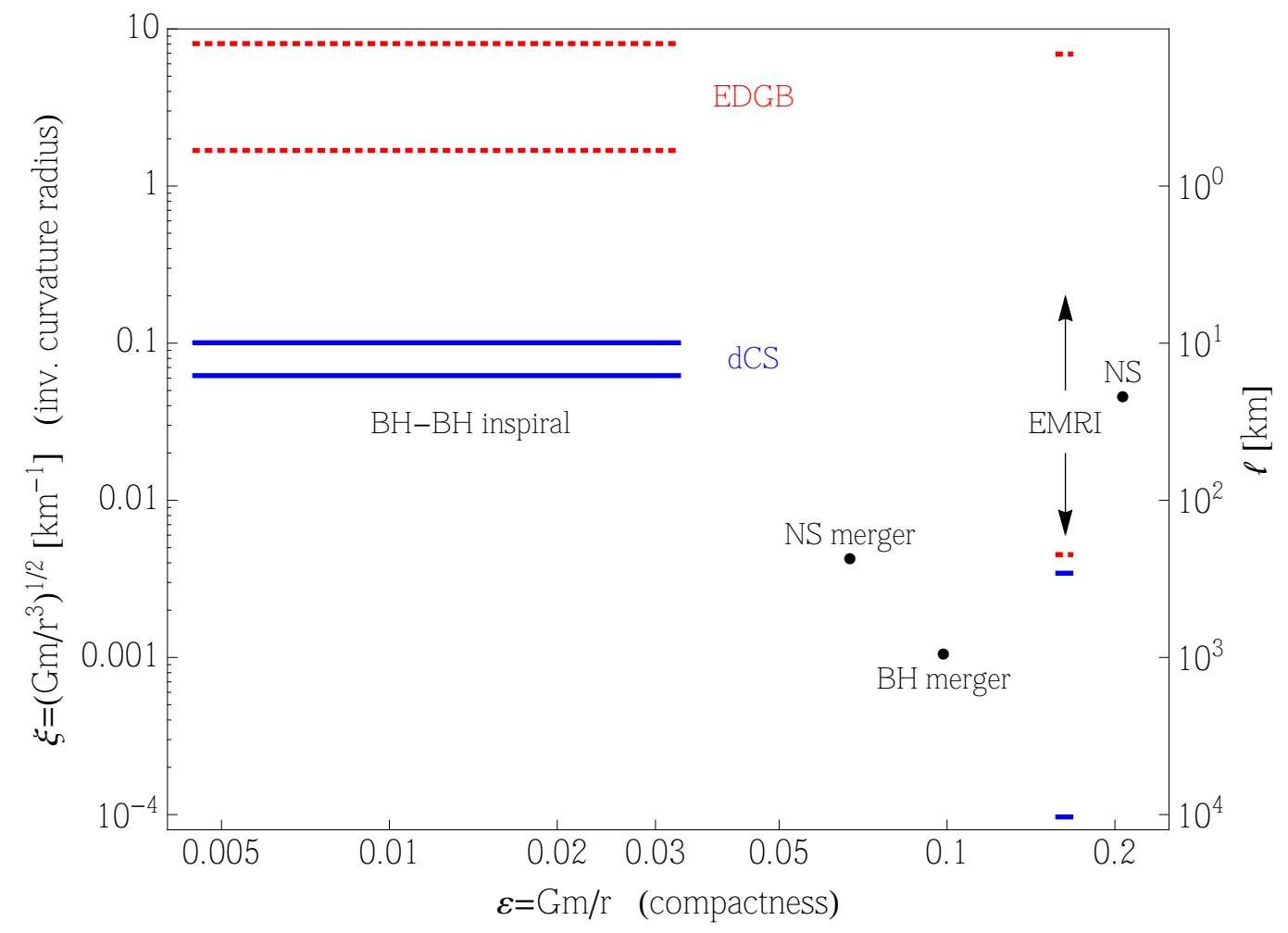

Figure 3. Estimated bounds on $\ell$ from GW measurements, shown by the dashed region in Fig. 2. We assume a GW detection at SNR 30 that is consistent with GR. Solid (blue) lines are for dCS, while dotted (red) lines are for EdGB. Estimated bounds from a $\mathrm{BH}-\mathrm{BH}$ inspiral in LIGO (an EMRI of a BH-BH binary in LISA) appear at left (right). The horizontal extent of such bounds represents the range of frequencies in band. Figure from [59].

\subsection{EMW Tests}

Let us now review BH based tests of GR with EMW observations [10,11]. We consider a $\mathrm{BH}$ with an accretion disk system. We cover X-ray continuum spectrum, Fe line emission, $\mathrm{BH}$ shadow and QPO in turn. We also comment on possible systematic errors in using these tests to probe GR. For simplicity, we will neglect the light bending effect in the explanation below. If one is to take such an effect into account, one needs to solve the null geodesic equations numerically using the ray-tracing algorithm [299,300]. Other possible tests that are not covered in this review include e.g. X-ray polarization [301,302], stars [192,303-307] and hot spots [308-310] around a black hole, and BH jets [311,312]. We refer the readers to e.g. [11] for more thorough review on these topics.

2.4.1. Continuum Spectrum A soft X-ray component of the generic spectrum of a stellar-mass $\mathrm{BH}$ with an accretion disk is interpreted as the thermal spectrum of an optically thick, geometrically thin disk. Such a disk is realized when the radiative efficiency is relatively large and the disk is described by the Novikov-Thorne model [313]. When the central object is the Kerr BH, the spectrum depends mainly on five parameters, 
the $\mathrm{BH}$ mass and spin, the inclination angle, the mass accretion rate and the distance from an observer. If one has independent measurements of the $\mathrm{BH}$ mass, distance and the inclination angle, one can apply the continuum fitting method to determine the BH spin (and the accretion rate). Such a method can also be used to distinguish the Kerr BH and a naked singularity [314,315]. On the other hand, if the central object is not the Kerr BH, the spectrum depends on additional parameters [114-122] that may be related to coupling constants in non-GR theories. Current observational data have already been used to place constraints on possible deviations from the Kerr BH [117, 121, 122].

The spectrum can be derived as follows. For an optically thick, geometrically thin accretion disk around a $\mathrm{BH}$, the time-averaged flux $\mathcal{F}(r)$ from the disk surface is given via the conservation of energy by $[117,313]$

$$
\mathcal{F}(r)=-\frac{\dot{M}}{4 \pi \sqrt{-\mathcal{G}}} \frac{\partial_{r} \Omega}{\left(E-\Omega L_{z}\right)^{2}} \int_{r_{\mathrm{in}}}^{r}\left(E-\Omega L_{z}\right)\left(\partial_{r} L_{z}\right) d r,
$$

where $r_{\text {in }}$ is the inner radius of the accretion disk, which is usually chosen to be the ISCO radius, $\dot{M}$ is the constant accretion rate that is determined from the mass conservation law and $\sqrt{-\mathcal{G}} \equiv \sqrt{N^{2} g_{r r} g_{\phi \phi}}$ with $N$ representing the lapse function. The choice of the inner radius is motivated from e.g. [316] when the source is in the high/soft state, though it is currently unclear whether such a choice is valid also in the low/hard state. $E, L_{z}$ and $\Omega$ are the specific energy, axial component of the specific angular momentum and the angular velocity for circular geodesics in the equatorial plane given by [117]

$$
\begin{aligned}
& \Omega=\frac{-\partial_{r} g_{t \phi} \pm \sqrt{\left(\partial_{r} g_{t \phi}\right)^{2}-\left(\partial_{r} g_{t t}\right)\left(\partial_{r} g_{\phi \phi}\right)}}{\partial_{r} g_{\phi \phi}}, \\
& E=-\frac{g_{t t}+g_{t \phi} \Omega}{\sqrt{-g_{t t}-2 g_{t \phi} \Omega-g_{\phi \phi} \Omega^{2}}}, \quad L_{z}=\frac{g_{t \phi}+g_{\phi \phi} \Omega}{\sqrt{-g_{t t}-2 g_{t \phi} \Omega-g_{\phi \phi} \Omega^{2}}},
\end{aligned}
$$

where $+(-)$ sign in $\Omega$ corresponds to the corotating (counterrotating) orbits. Assuming a black body radiation, the effective temperature is determined from $\mathcal{F}$ by $\mathcal{F}=\sigma T^{4}$, where $\sigma$ is the Stefan-Boltzmann constant. Neglecting the light bending effect, one finds the luminosity as

$$
L(\nu)=8 \pi h \cos i \int_{r_{\text {in }}}^{r_{\text {out }}} \int_{0}^{2 \pi} \frac{\nu^{3} \sqrt{-\mathcal{G}}}{\exp \left[h \nu_{e} / k T\right]-1} d \phi d r,
$$

where $h$ is the Planck constant, $k$ is the Boltzmann constant, $i$ is the inclination angle and $r_{\text {out }}$ is the outer radius of the accretion disk. $\nu$ and $\nu_{e}$ are the frequencies in the local rest frame of the observer and the emitter respectively, which are related through the redshift factor $g$ by

$$
g \equiv \frac{\nu}{\nu_{e}}=\frac{\sqrt{-g_{t t}-2 g_{t \phi} \Omega-g_{\phi \phi} \Omega^{2}}}{1+\Omega r \sin \phi \sin i} .
$$

Such a factor encodes the frame dragging, the gravitational redshift and the special and general relativistic effects of Doppler boost. 
2.4.2. Iron Line Emission Another way to test GR is to use the iron line emission from an accretion disk around a stellar-mass or super-massive BH. Thermal photons are scattered by electrons (the inverse Compton scattering) in a hot corona above the disk, which produces a power-law component in the X-ray spectrum. Such photons irradiate the disk and produces spectral lines by fluorescence, the strongest of which is the $\mathrm{K} \alpha$ iron line at $6.4 \mathrm{keV}$ (this becomes roughly $7.1 \mathrm{keV}$ for the fully ionized iron line). This line is narrow in frequency intrinsically, but is broadened and skewed due to relativistic effects. The existence of such a line was detected in e.g. [317,318] (see [319,320] for reviews).

For a Kerr BH, the shape of the emission spectrum depends on the BH spin, the inclination angle, the disk emissivity and the outer radius of the emission region. In particular, the lower-energy tail depends on the inner radius of the disk, which is again commonly identified as the ISCO radius. Since such a radius depends on the BH spin in units of the $\mathrm{BH}$ mass, one can measure the dimensionless $\mathrm{BH}$ spin from the shape of the line profile even if the $\mathrm{BH}$ mass and distance are not measured. One can also use such a profile to test the BH geometry, as studied in [118-120,123-128,321]. The continuum spectrum method cannot be applied to an accretion disk around a super-massive BH because the spectrum lies in the ultra-violet (UV) band, which is difficult to measure due to dust absorption, while the iron line method can be applied to both stellar-mass and super-massive BHs.

The iron line emission profile can be calculated as follows. In the celestial coordinates $(\alpha, \beta)$ in the observer's plane in the sky, the observed flux at energy $E$ is given by $[124,321]$

$$
\mathcal{F}(E)=\frac{1}{d^{2}} \iint g^{3} I_{e}\left(E_{e}\right) d \alpha d \beta
$$

where $d$ is the distance between the observer and the $\mathrm{BH}$ and the redshift factor $g$ is given by Eq. (76). $I_{e}\left(E_{e}\right)$ is the emitted specific intensity at the emitted energy $E_{e}$ given by $[124,321]$

$$
I_{e}\left(E_{e}\right)=r^{-q} \delta\left(E_{e}-E_{\mathrm{K} \alpha}\right)=r^{-q} g \delta\left(E-g E_{\mathrm{K} \alpha}\right),
$$

where $E_{\mathrm{K} \alpha}=7.1 \mathrm{keV}$ for fully ionized iron lines and $q(>0)$ is the emissivity index. Neglecting the light-bending effect, which is a good approximation for emission from a small inclination (face-on) disk, one can convert the integral over $(\alpha, \beta)$ in Eq. (77) to that over $(r, \phi)$ as [321,322]

$$
\mathcal{F}(E)=\frac{\cos i}{d^{2}} \int_{0}^{2 \pi} \int_{r_{\mathrm{ISCO}}}^{r_{\mathrm{out}}} g^{4} r^{-q} \delta\left(E-g E_{\mathrm{K} \alpha}\right) \sqrt{-\mathcal{G}} d r d \phi
$$

where $i$ is the inclination angle and $r_{\text {out }}$ is the outer radius of the emission region in the disk.

2.4.3. BH Shadow VLBI at (sub-)millimeter wavelengths will allow us to directly image $\mathrm{BHs}$ in the near future using e.g. Event Horizon Telescope. Due to strong gravity in the vicinity of a $\mathrm{BH}$, some light rays emitted from an accretion disk terminates inside the $\mathrm{BH}$ 
event horizon and never reaches an observer at infinity. This, in turn, produces a dark region in the image, which is called a BH shadow. Such a shadow within GR has been calculated by many authors [323-333]. Since the shape of a shadow depends strongly on the $\mathrm{BH}$ geometry, one can in principle measure not only the $\mathrm{BH}$ spin but also a possible deviation from the Kerr BH [119, 129-145,334], which can be used to test GR.

The boundary of the BH shadow (a photon ring) is determined from an unstable photon orbit with a constant radius. Such a boundary for the Kerr BH can be calculated as follows [329]. The existence of the Carter constant $\mathcal{Q}$ allows one to separate the null geodesic equations. In particular, the radial component of the equations in the Boyer-Lindquist coordinate is given by

$$
\rho^{2} \frac{d r}{d \lambda}= \pm \sqrt{\mathcal{R}}, \quad \mathcal{R} \equiv\left(r^{2}+a^{2}-a \xi\right)^{2}-\Delta \mathcal{I}
$$

where $\rho^{2} \equiv r^{2}+a^{2} \cos ^{2} \theta, \Delta \equiv r^{2}-2 M r+a^{2}, \lambda$ is the affine parameter and $M$ and $a$ are the BH mass and Kerr parameter respectively. $\mathcal{I}$ is a function of $\tilde{\xi} \equiv L_{z} / E$ and $\tilde{\eta} \equiv \mathcal{Q} / E$ given by $\mathcal{I} \equiv \tilde{\eta}+(a-\tilde{\xi})^{2}$ with $E$ and $L_{z}$ representing the specific energy and the axial component of the specific angular momentum of a photon orbit. The conditions for the unstable photon orbit are given by

$$
\mathcal{R}=0, \quad \frac{d \mathcal{R}}{d r}=0,
$$

which can be solved for $\tilde{\xi}$ and $\tilde{\eta}$ to yield $*$

$$
\tilde{\xi}=\frac{r^{2}+a^{2}}{a}-2 \frac{r \Delta}{a(r-M)}, \quad \tilde{\eta}=-\frac{r^{3}\left[r(r-3 M)^{2}-4 a^{2} M\right]}{a^{2}(r-M)^{2}} .
$$

The photon ring in the celestial coordinate $(\alpha, \beta)$ that an observer sees is given by

$$
\alpha=-\lim _{r \rightarrow \infty} \frac{r p^{(\phi)}}{p^{(t)}}=-\tilde{\xi} \csc i, \quad \beta=\lim _{r \rightarrow \infty} \frac{r p^{(\theta)}}{p^{(t)}}=\sqrt{\tilde{\eta}+a^{2} \cos ^{2} i-\tilde{\xi}^{2} \cot ^{2} i},
$$

where $i$ is the inclination angle and $\left(p^{(t)}, p^{(r)}, p^{(\theta)}, p^{(\phi)}\right)$ are the tetrad components of the photon momentum in reference frames that are locally nonspinning.

Figure 4 presents the photon ring for the Kerr BH with $i=\pi / 2$ and various spin parameters $\chi=a / M$. Observe that the center of the ring shifts to positive $\alpha$ and the deviation in the shape from a circle becomes larger as one increases the spin. This shows that one can extract the information of the $\mathrm{BH}$ spin from the shape of the $\mathrm{BH}$ shadow provided that one can break the degeneracy between the spin and inclination. BH spacetimes in non-GR theories in general do not admit the Carter constant. (See e.g. [260] for a concrete example). In such cases, one cannot separate the null geodesic equations and one needs to solve them numerically using the ray-tracing algorithm [299, 300].

* $\overline{\tilde{\xi}}$ on the equatorial plane is related to the real part of the QNM frequency of the central BH within the WKB approximation [335,336]. 


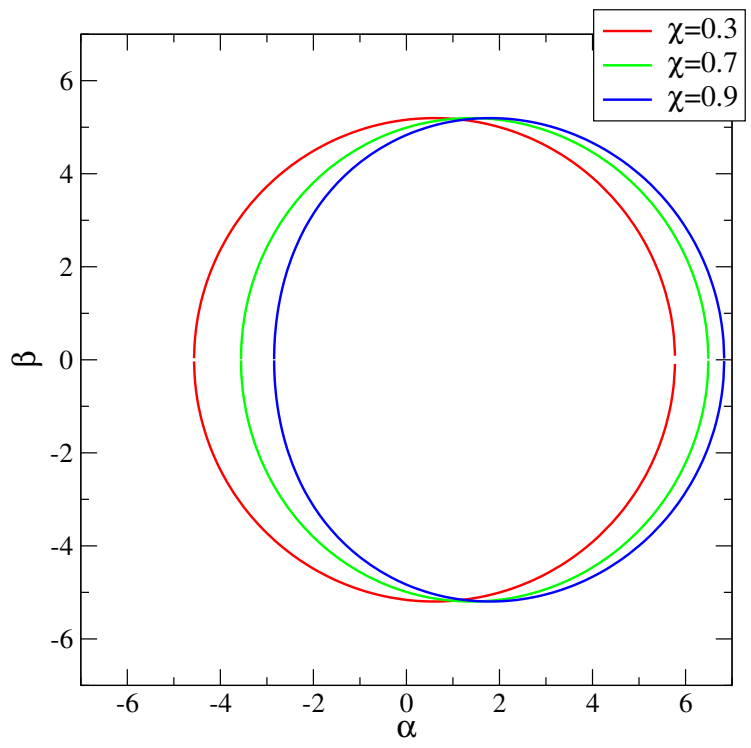

Figure 4. (Color online) Photon ring of a Kerr BH with an inclination angle of $\pi / 2$ and with various dimensionless spin parameter $\chi$. Observe how the shape changes as one varies $\chi$.

2.4.4. Quasi-periodic Oscillation Observations of LMXBs show peaked features in the power density spectra called QPOs (see Refs. [337,338] for reviews). In particular, high frequency QPOs from a BH-accretion disk system are observed in commensurable pairs with the ratio 3:2 [337,339]. Although the origin of such QPOs is unclear, many models exist and they usually relate QPO frequencies to fundamental oscillation frequencies of a test particle orbiting a central object, namely the Keplerian and epicyclic frequencies.

Such fundamental frequencies can be calculated as follows. First, from the normalization condition $u^{\mu} u_{\mu}=-1$ of the 4-velocity $u^{\mu}$ of a test particle, one finds

$$
g_{r r} \dot{r}^{2}+g_{\theta \theta} \dot{\theta}^{2}=V_{\mathrm{eff}}, \quad V_{\mathrm{eff}} \equiv \frac{E^{2} g_{\phi \phi}+2 E L_{z} g_{t \phi}+L_{z}^{2} g_{t t}}{g_{t \phi}^{2}-g_{t t} g_{\phi \phi}}-1,
$$

where the over dots refer to derivatives with respect to an affine parameter. The conditions for a circular orbit in the equatorial plane, $V_{\text {eff }}=0, \partial_{r} V_{\text {eff }}=0$ and $\partial_{\theta} V_{\text {eff }}=0$ give $\Omega, E$ and $L_{z}$ shown in Eqs. (73) and (74). The Keplerian frequency is given by $\nu_{\phi}=\Omega /(2 \pi)$. Next, let us consider small perturbations along the radial $\left(\delta_{r}\right)$ and polar $\left(\delta_{\theta}\right)$ directions from a fiducial orbit with $r=r_{0}$ and $\theta=\pi / 2$. Linear order perturbations are given by the solutions to

$$
\frac{d^{2} \delta_{r}}{d t^{2}}+\Omega_{r}^{2} \delta_{r}=0, \quad \frac{d^{2} \delta_{\theta}}{d t^{2}}+\Omega_{\theta}^{2} \delta_{\theta}=0,
$$

where

$$
\Omega_{r}^{2}=-\frac{1}{2 g_{r r}\left(u^{t}\right)^{2}} \frac{\partial^{2} V_{\mathrm{eff}}}{\partial r^{2}}, \quad \Omega_{\theta}^{2}=-\frac{1}{2 g_{\theta \theta}\left(u^{t}\right)^{2}} \frac{\partial^{2} V_{\mathrm{eff}}}{\partial \theta^{2}} .
$$


The radial and vertical epicyclic frequencies are given by $\nu_{r}=\Omega_{r} /(2 \pi)$ and $\nu_{\theta}=\Omega_{\theta} /(2 \pi)$ respectively. For the Kerr BH, the fundamental frequencies are given by [340,341]

$$
\begin{aligned}
& \nu_{\phi}=\frac{1}{2 \pi} \frac{M^{1 / 2}}{r^{3 / 2} \pm a M^{1 / 2}}, \\
& \nu_{r}=\nu_{\phi}\left(1-\frac{6 M}{r} \pm \frac{8 a M^{1 / 2}}{r^{3 / 2}}-\frac{3 a^{2}}{r^{2}}\right), \\
& \nu_{\theta}=\nu_{\phi}\left(1 \mp \frac{4 a M^{1 / 2}}{r^{3 / 2}}+\frac{3 a^{2}}{r^{2}}\right),
\end{aligned}
$$

where the upper (lower) sign corresponds to a corotating (counterrotating) orbit.

We now review various QPO models. We classify them into three classes [342], (i) kinematic, (ii) resonant and (iii) diskoseismic models. We summarize the upper and lower $\mathrm{kHz}$ QPO frequencies in each model in the kinematic and resonant models in Table 2. These QPO models were applied to probe possible deviations from the Kerr $\mathrm{BH}$ in [119, 146-149,343].

Kinematic Models: These models consider hot spots or blobs orbiting in the accretion disks around the central compact objects as the origin of QPOs. One example is the relativistic precession model [344-346], which was first proposed to explain the QPOs for NSs in LMXBs and was applied to those for stellar-mass BHs in [347]. The higher and lower $\mathrm{kHz}$ QPOs in such a model are given by $\nu_{\phi}$ and $\nu_{\phi}-\nu_{r}$, where the latter corresponds to the periastron precession frequency. Such a model also explains the low-frequency QPO as $\nu_{\phi}-\nu_{\theta}$, which corresponds to the nodal precession frequency. These triplets QPOs were observed in GRO J1655-40 [348-350] with the Rossi X-ray Timing Explorer (RXTE). The mass derived from such a QPO model [351] is consistent with that from optical/near-infrared spectro-photometric observations [352], while the spin measurement [351] is inconsistent with that from continuum spectrum fitting [353]. Another kinematic model is the tidal disruption model [354,355], in which QPOs are caused by a blob of inhomogeneity inside the accretion disk being tidally stretched.

Resonant Models: Commensurability of QPO frequencies arises more naturally in the resonant models. Resonance models [339,340,356,357] promote Eq. (85) by introducing nonlinear couplings $F_{r}$ and $F_{\theta}$ between two epicyclic modes on the right hand side:

$$
\frac{d^{2} \delta_{r}}{d t^{2}}+\Omega_{r}^{2} \delta_{r}=\Omega_{r}^{2} F_{r}\left(\delta_{r}, \delta_{\theta}, \dot{\delta}_{r}, \dot{\delta}_{\theta}\right), \quad \frac{d^{2} \delta_{\theta}}{d t^{2}}+\Omega_{\theta}^{2} \delta_{\theta}=\Omega_{\theta}^{2} F_{\theta}\left(\delta_{r}, \delta_{\theta}, \dot{\delta}_{r}, \dot{\delta}_{\theta}\right)
$$

where the dot refers to the time derivative. The parametric resonance model $[356,357]$ is given by $F_{r}=0$ and $F_{\theta}=-\delta_{r} \delta_{\theta}$. In such a case, the equation for $\delta_{\theta}$ becomes the Mathieu equation and the ratio between $\nu_{r}$ and $\nu_{\theta}$ is given by $\nu_{r} / \nu_{\theta}=2 / n$, where $n$ is a positive integer. For the Kerr background, $\nu_{\theta}>\nu_{r}$, and hence $n=3$ gives the dominant contribution. $n=1$ and $n=2$ modes may arise in non-Kerr backgrounds. The forced resonance model [357] corresponds to $F_{r}=0$ and $F_{\theta}=\delta_{r} / \Omega_{\theta}^{2}+\bar{F}_{\theta}\left(\delta_{\theta}\right)$ where $\bar{F}_{\theta}$ represents the nonlinear term in $\delta_{\theta}$. Such a nonlinear term gives resonant solutions for $\delta_{\theta}$, with the simplest frequencies being $\nu_{+}=\nu_{\theta}+\nu_{r}$ and $\nu_{-}=\nu_{\theta}-\nu_{r}$. The 
Table 2. Upper and lower $\mathrm{kHz}$ QPO frequencies in various models. The first two models correspond to the kinematic models while other models correspond to the resonant models. (See also Table 1 of [342].) $\nu_{\phi}$ is the Keplerian frequency while $\nu_{r}$ and $\nu_{\theta}$ are the radial and vertical epicyclic frequencies respectively. $\nu_{+}$and $\nu_{-}$are defined by $\nu_{+}=\nu_{\theta}+\nu_{r}$ and $\nu_{-}=\nu_{\theta}-\nu_{r}$.

\begin{tabular}{llll}
\hline \hline Models & References & lower frequency & upper frequency \\
\hline relativistic precession & {$[344-346]$} & $\nu_{\phi}-\nu_{r}$ & $\nu_{\phi}$ \\
tidal disruption & {$[354,355]$} & $\nu_{\phi}$ & $\nu_{\phi}+\nu_{r}$ \\
\hline parametric resonance & {$[356,357]$} & $\nu_{r}$ & $\nu_{\theta}$ \\
forced resonance & {$[357]$} & $\nu_{-}$ & $\nu_{\theta}$ \\
& {$[357]$} & $\nu_{\theta}$ & $\nu_{+}$ \\
Keplerian resonance & {$[357]$} & $\nu_{r}$ & $\nu_{\phi}$ \\
& {$[357]$} & $2 \nu_{r}$ & $\nu_{\phi}$ \\
warped disk oscillation & {$[357]$} & $\nu_{\phi}$ & $3 \nu_{r}$ \\
non-axisymmetric disc-oscillation & {$[358-362]$} & $2\left(\nu_{\phi}-\nu_{r}\right)$ & $2 \nu_{\phi}-\nu_{r}$ \\
& {$[363]$} & $\nu_{\phi}-\nu_{r}$ & $\nu_{\theta}$ \\
& {$[364]$} & $\nu_{\phi}-\nu_{r}$ & $2 \nu_{\phi}-\nu_{\theta}$ \\
\hline \hline
\end{tabular}

Keplerian [357] resonance model predicts couplings between Keplerian frequency and an epicyclic frequency, although such a model maybe more unrealistic than the parametric or forced resonance model. Kato [358-362] proposed the warped disk oscillation models, where QPOs are caused by several resonances in a deformed disk with somewhat exotic geometry. Other models include the non-axisymmetric disc-oscillation models proposed in $[363,364]$, where the frequencies of oscillation modes of a disk is similar to frequencies predicted in the relativistic precession model. Although the resonant models can naturally explain commensurability of QPO frequencies, the underlying mechanisms are unclear in most of the models.

Discoseismic Models: These models predict that QPO frequencies correspond to those of the fundamental diskoseismic modes [365-372], namely the g-modes, c-modes and p-modes. The g-modes are inertial gravity wave modes that occur at frequencies close to the maximum radial epicyclic frequency near the inner edge of the disc [368]. The c-modes are corrugation modes that exist only in disks that corotate with the central $\mathrm{BH}$ and whose frequency coincides with the Lense-Thirring frequency at a certain radius close to the BH ISCO [369]. The p-modes are inertial-pressure oscillation modes that arises close to the edge of the accretion disc. Unfortunately, magnetohydrodynamic simulations show that such modes typically damp due to various instabilities [373-375] and do not reproduce the 3:2 QPOs.

2.4.5. Systematics We will now comment on possible systematic errors that may deteriorate tests of GR with EMW observations. One major origin of such systematics is the uncertainty in the accretion disk model. For example, both the continuum spectrum and iron line methods assume that the disk is geometrically thin and optically thick. However, recent quasar observations with foreground microlensing are not fully consistent 
with the standard geometrically thin disk model [376-383], and the finite disk size may produce systematic errors [384]. The BH shadow observations are also affected by the uncertainty in the disk model. Although the photon ring (Fig. 4) does not depend on the disk model, the intensity map of the image does depend on such a model.

The continuum spectrum method requires the disk inclination is known independently [385], and one way is to simply assume that the disk is aligned with the BH spin. On one hand, such an alignment is supported by calculations of binary population synthesis [386]. However, the time scale for the Bardeen-Peterson effect [387] to complete seems to be too long [388], and both observational data [389] and theoretical calculations [390] suggest that tilted disks may exist. The observational data for XTE J1550-564 [391] shows a good alignment, while those for GRO J1655-40 [392,393] and V4641 Sgr [394] infer misalignment. The continuum spectrum method mentioned in Sec. 2.4.1 assumed that the radiation is a blackbody, which is not completely true in nature, and one needs to correct the temperature by introducing a hardening factor (or a color factor). The correct computation of such a factor requires an accurate modeling of the disk atmosphere [395], and significant progress has been made in e.g. [385, 396].

The iron line method requires the knowledge of the emissivity, which was taken as a simple power-law in Sec. 2.4.2. However, this is clearly an approximation, and one may need a better modeling in order to reduce systematic errors. For example, a cutoff in the emissivity may exist near ISCO, and its position is likely to affect the measurement accuracy of the spin and deviations from Kerr [397-399]. Also, the assumption of taking the inner radius of the disk to be ISCO is not fully justified, as BHs are typically in the low/hard state. Furthermore, the thermal component of the disk may overlap with the low energy tail of the iron line, which makes the modeling more complicating. One may also need to account for Compton broadening [11].

The QPO method is affected by the uncertainty in identifying the correct QPO model. Namely, one can carry out tests of GR only if the correct QPO model is known a priori. Furthermore, current models are incomplete, as they cannot explain e.g. the energy dependence of QPOs [384]. They also cannot explain why some sources show QPOs with a 3:2 frequency ratio, while many sources exist with no high frequency QPOs.

\section{Scalar-Tensor Theories}

We now look at specific alternative theories of gravity in turn. First, we will focus on scalar-tensor theories.

\subsection{Basics}

We here review basic properties of scalar-tensor theories, BH solutions and current constraints from solar system and binary pulsar observations. 
3.1.1. Theories Scalar-tensor theories are one of the simplest and most well-studied alternative theories of gravity [162]. One introduces one (or more) additional scalar field to GR. Such a scalar field can explain the current accelerating expansion of the Universe [165] or inflation [166], and it also arises from low energy effective theory of string theory $[163,164]$ as a dilaton.

After an appropriate field redefinition, the most general action for scalar-tensor theories with a single scalar field in the Jordan frame, with at most second derivatives of the fields up to total derivatives* is given by [402,403]

$$
S=\frac{1}{16 \pi} \int d^{4} x \sqrt{-g}\left[\vartheta R-\frac{\omega(\vartheta)}{\vartheta} g^{\mu \nu}\left(\partial_{\mu} \vartheta\right)\left(\partial_{\nu} \vartheta\right)+V(\vartheta)+16 \pi \mathcal{L}_{\text {mat }}(\Psi)\right] .
$$

Here, $g_{\mu \nu}, g$ and $R$ are the metric, its determinant and the Ricci scalar in the Jordan frame, $\vartheta$ and $V$ represent the scalar field and its potential, while $\mathcal{L}_{\text {mat }}$ corresponds to the matter Lagrangian with $\Psi$ representing the matter Lagrangian density. The coefficient $\omega(\vartheta)$ in front of the kinetic term of the scalar field is a function of $\vartheta$ that characterizes the theories. When $\omega(\vartheta)=\omega_{\mathrm{BD}}$ and $V(\vartheta)=0$ with $\omega_{\mathrm{BD}}$ a constant called the Brans-Dicke (BD) parameter, the action reduces to that of massless BD theory [404]. The inverse of $\omega_{\mathrm{BD}}$ characterizes the strength of a coupling between the scalar and matter field. The theory reduces to GR in the limit $\omega_{\mathrm{BD}} \rightarrow \infty$. On the other hand, if one assumes $V(\vartheta)=(1 / 2) m_{s} \vartheta^{2}$, the theory reduces to massive BD theory $[22,188]$ with $m_{s}$ representing the mass of the scalar field. The scalar-tensor theories with multiple scalar fields are studied in [405].

Other well-studied scalar-tensor theories include quasi BD theory proposed by Damour and Esposito-Farèse [406,407]. Let us first move to the Einstein frame by redefining the scalar field as $\vartheta=\vartheta(\varphi)$ and carrying out a conformal transformation on the metric as $g_{\mu \nu} \rightarrow \tilde{g}_{\mu \nu} \equiv A(\varphi)^{-2} g_{\mu \nu}$ with $A(\varphi)=\vartheta^{-1 / 2}$. In such a frame, the action is given by

$$
S=\frac{1}{16 \pi} \int d^{4} x \sqrt{-\tilde{g}}\left\{\tilde{R}-2 \tilde{g}^{\mu \nu}\left(\partial_{\mu} \varphi\right)\left(\partial_{\nu} \varphi\right)+U(\varphi)+16 \pi \mathcal{L}_{\mathrm{mat}}\left[\Psi, A(\varphi) \tilde{g}_{\mu \nu}\right]\right\},
$$

where $\tilde{g}$ and $\tilde{R}$ are the determinant of the metric and the Ricci scalar in the Einstein frame while $U(\varphi)=A(\varphi)^{4} V(\vartheta)$ is the potential for the scalar field in this frame. Notice that the scalar field is now minimally coupled to the metric. The relation between $\omega(\vartheta)$ and $A(\varphi)$ is given by [2]

$$
\omega(\vartheta)=\frac{1-3 \alpha(\varphi)^{2}}{2 \alpha(\varphi)^{2}}, \quad \alpha(\varphi) \equiv \frac{d \ln A(\varphi)}{d \varphi} .
$$

Damour and Esposito-Farèse [406,407] set $U=0$ and expanded $\alpha(\varphi)$ around a constant $\varphi_{0}$ at spatial infinity as

$$
\alpha(\varphi)=\alpha_{0}+\beta_{0}\left(\varphi-\varphi_{0}\right)+\mathcal{O}\left[\left(\varphi-\varphi_{0}\right)^{2}\right],
$$

* The most general scalar-tensor theory with a single scalar field and second-order field equations is Horndeski's theory [400], whose action can be expressed in terms of Galileon interactions [401]. 
where $\alpha_{0}$ and $\beta_{0}$ are constants. In fact, having two constants $\alpha_{0}$ and $\varphi_{0}$ are redundant as they both enter only in the constant term up to the above truncation. For example, one can choose $\varphi_{0}=\alpha_{0} / \beta_{0}$ such that the constant term vanishes and $\alpha(\varphi)=\beta_{0} \varphi+\mathcal{O}\left[\left(\varphi-\varphi_{0}\right)^{2}\right][180,181]$.

3.1.2. BH Solutions We now briefly describe BH solutions in scalar-tensor theories (see Sec. 3.1 of [189] for a recent review on this topic). As in GR, no-hair theorem exists for stationary, isolated BHs in vacuum in scalar-tensor theories with real, time-independent scalars [167-171]. This is because in the Einstein frame in vacuum, the scalar-tensor theory reduces to GR with a minimally coupled scalar field. Such an analysis has recently been extended to stationary BHs with a single, real, time-dependent scalar field in [172]. On the other hand, Jacobson [408] perturbatively showed that a BH can acquire a scalar hair if both the metric and the scalar field are time dependent. Regarding BH solutions with a complex scalar field, Pena and Sudarsky [409] showed that a spherically symmetric BH solution does not exist, but BH solutions with a rotating configuration have been constructed numerically in [410-412] (see [413] for a recent review). If a BH is surrounded by matter, Cardoso et al. [414,415] showed that it can spontaneously acquire a scalar hair due to a tachyon instability, similar to spontaneous scalarization for neutron stars [406, 407].

In Horndeski's theory, Hui and Nicolis [173] extended Refs. [167,171] and claimed that if the scalar field enjoys a shift symmetry, a static, spherically symmetric, asymptotically flat BH in vacuum cannot have a scalar hair. Sotiriou and Zhou [177,178] pointed out that an exceptional case exists in this claim, which is when a scalar field is linearly coupled to the Gauss-Bonnet invariant density. The static, spherically symmetric, asymptotically flat $\mathrm{BH}$ solution in such a theory were previously constructed in [175, 176]. Babichev and Charmousis extended Ref. [416] to Horndeski's theory and showed that BHs can acquire a scalar hair with a time-dependent scalar field. Linear perturbations of static, spherically symmetric BHs in Horndeski's theory in both even and odd parity sectors and necessary conditions for their stability were studied in $[417,418]$.

3.1.3. Curent Constraints We here review current constraints on scalar-tensor theories. A strong bound on such theories has been placed by the Cassini satellite while on its way to Saturn [190]. In the PN gauge, the metric suitable for the solar system is given by $[1,2]$

$$
d s^{2}=-(1-2 \Phi) d t^{2}+(1-2 \gamma \Phi) \delta_{i j} d x^{i} d x^{j}+\mathcal{O}\left(\epsilon^{3 / 2}\right)
$$

where the dimensionless parameter $\epsilon$ is defined via $\epsilon \equiv M_{\odot} / r$ with $r$ representing the typical length scale and $\Phi=-M_{\odot} / r+\mathcal{O}\left(\epsilon^{2}\right)$ is the gravitational potential. Cassini placed a bound on $\gamma$ by measuring the time delay that occurs when the light from the satellite is deflected as it passes near the Sun (Shapiro time delay). Cassini found that $|\gamma-1|$ must be within $\sim 10^{-5}$, which corresponds to a bound on the massless BD theory as $\omega_{\mathrm{BD}}>4 \times 10^{4}$. The bound on massive BD theory using the Shapiro time delay measurement is derived in $[188,191]$. 
Another strong bound comes from binary pulsar observations. Since the dipolar radiation is suppressed for NS/NS binaries, stronger bounds have been placed from the orbital decay rate measurement of NS/white dwarf (WD) binaries, such as PSR J1141-6545 [185], PSR J1738+0333 [186] and PSR J0348+0432 [187]. For example PSR J1738+0333 [186] places a bound on massless BD theory that is comparable to the solar system bound. Such binary pulsar constraints place stronger bounds on some parameter region in quasi $\mathrm{BD}$ theory than the solar system one. The bound on the massive BD theory from such observations have been derived in [188].

\section{2. $G W$ Tests}

We now explain future projected constraints on scalar-tensor theories with GW observations.

3.2.1. Massless BD Theory Let us first review how one can derive corrections to GWs emitted from a compact binary in massless BD theory. Will [419] derived the scalar field in a region far from the binary. He found that the perturbation to the scalar field $\tilde{\vartheta}$ from the background is given by

$$
\tilde{\vartheta}=-\frac{4}{3+2 \omega_{\mathrm{BD}}} \eta \frac{m}{r} \mathcal{S} \boldsymbol{n} \cdot \boldsymbol{v}+\mathcal{O}\left(\frac{m^{2}}{r^{2}}\right),
$$

where $r$ is a distance from the binary to the field point, $\boldsymbol{v}$ is the velocity of the binary constituents and $\boldsymbol{n}$ is the unit vector from the binary to the field point. We recall that $\eta$ is the symmetric mass ratio and $m$ is the total mass of the binary. $\mathcal{S}$ is given by

$$
\mathcal{S} \equiv s_{1}-s_{2}, \quad s_{a} \equiv-\left[\frac{\partial\left(\ln m_{a}\right)}{\partial(\ln G)}\right]_{\infty} .
$$

Here, $s$ is the sensitivity which is related to the scalar charge and the subscript $\infty$ shows that the quantity inside the square brackets is to be evaluated at spatial infinity. Notice that in BD theory, the gravitational constant $G$ depends on the scalar field. $s$ is roughly given by the object's compactness, so $s \sim 0.2$ for NSs and $s=0.5$ for BHs. Will and Zaglauer [420] then calculated the energy flux emitted from a binary. Keeping to leading order in the BD correction in terms of PN order, such a flux is given by

$$
\frac{d E_{\mathrm{GW}}}{d t}=-\frac{32}{5}\left\langle\frac{\eta^{2} m^{4}}{r_{12}^{4}} v^{2}\left[1+\frac{5}{48} \mathcal{S}^{2} \bar{\omega} v^{-2}+\mathcal{O}\left(v^{2}\right)\right]\right\rangle,
$$

where $r_{12}$ is the binary separation, $\bar{\omega} \equiv 1 / \omega_{\mathrm{BD}}$ and the angular brackets represent the orbital average. Based on the energy flux above, Will [13] derived the gravitational waveform of a compact binary in this theory. The phase in Fourier space is given by that in the PPE waveform in Eq. (32) with

$$
\beta_{\mathrm{ppE}}=-\frac{5}{3584} \eta^{2 / 5} \mathcal{S}^{2} \bar{\omega}, \quad b_{\mathrm{ppE}}=-\frac{7}{3} .
$$


This BD correction in the waveform phase is of " $-1 \mathrm{PN}$ " order relative to the leading GR contribution.

Regarding higher PN corrections, Mirshekari and Will [421] derived the equations of motion of a compact binary in scalar-tensor theories with a single scalar field and a vanishing potential up to $2.5 \mathrm{PN}$ order. They showed that the radiation reaction enters at 1.5PN order due to the scalar dipolar radiation, which is $1 \mathrm{PN}$ order lower than that in GR due to quadrupolar radiation. For BH binaries, such equations of motion are exactly the same as those in GR to 2.5PN order. Yunes et al. [23] showed that the equations of motion for BH EMRIs are the same as those in GR to all PN orders in the small mass ratio limit. This can be interpreted as a generalization of the $\mathrm{BH}$ no-hair theorem in scalar-tensor theories to a BH binary system. The gravitational and scalar radiation are calculated to $2 \mathrm{PN}$ [422] and to $1.5 \mathrm{PN}$ [423] orders respectively. Then, Lang [423] derived the total energy flux carried away to infinity to $1 \mathrm{PN}$ order.

We now review proposed constraints on massless BD theory from future GW observations with NS/BH binaries. The results are summarized in Tables 3 and 4. The bound on $\omega_{\mathrm{BD}}$ using GWs from compact binaries was first studied by Eardley [424]. Will [13] derived the BD correction to the gravitational waveform phase from compact binaries as mentioned above and carried out a matches filtering analysis with Adv. LIGO. Scharre and Will [15] and Will and Yunes [16] performed a similar analysis using LISA. In particular, Will and Yunes improved previous work by studying how the bounds on $\omega_{\mathrm{BD}}$ depend on LISA's various noise sources, such as position and acceleration noises. They also studied how the bounds change as a function of the LISA's arm lengths. Berti et al. [17] calculated the measurement accuracy in $\omega_{\mathrm{BD}}$ with LISA, taking the effect of spin-orbit coupling into account. They also carried out a Monte Carlo simulation, where they randomly distributed $10^{4}$ sources over the sky, and derived an averaged bound on $\omega_{\mathrm{BD}}$. They found that LISA can place the proposed bound of $\omega_{\mathrm{BD}}>10799$ on average with a $1 \mathrm{yr}$ observation of GWs emitted from a $(1.4+1000) \mathrm{M}_{\odot} \mathrm{BH} / \mathrm{NS}$ binary with an SNR of $\sqrt{200}$.

Yagi and Tanaka [18] extended previous analyses by carrying out a Monte Carlo simulation to estimate proposed bounds on massless BD theory using LISA, taking both the spin precession and eccentricity of a binary system into account. They found that the inclusion of eccentricity weakens the bound by $4-5$ times than the one without including eccentricity. This is because both $\omega_{\mathrm{BD}}$ and eccentricity enter at a negative PN order in the gravitational waveform phase relative to the leading GR term, which results in a strong correlation between these two parameters. One can break the degeneracy by imposing prior information on eccentricity. Figure 5 presents the probability distribution of the lower bound on $\omega_{\mathrm{BD}}$, assuming LISA detects GW signals from $(1.4+1000) \mathrm{M}_{\odot}$ $\mathrm{BH} / \mathrm{NS}$ binaries with a circular orbit and an SNR of $\sqrt{200}$. Observe that the bound is weaker than the current solar system bound. In [19], Yagi and Tanaka performed a similar analysis using DECIGO/BBO. The results are also shown in Fig. 5, where the authors assumed that DECIGO/BBO detects GW signals from $(1.4+10) \mathrm{M}_{\odot} \mathrm{BH} / \mathrm{NS}$ binaries with a circular orbit and an SNR of $\sqrt{200}$. Observe that the proposed constraints 
Table 3. Summary of previous work on probing BD theory with GWs from NS/BH binaries. The second column represents the maximum PN order that is taken into account. The third, fourth and fifth columns present whether the spin-orbital coupling $\beta$, spin precession and eccentricity are included respectively. The sixth column shows whether each work considers a detection from multiple sources. The seventh column describes a type of analyses being performed (either the pattern-averaged (PA) or Monte Carlo (MC).) This table is taken from [21].

\begin{tabular}{lllllll}
\hline \hline Reference & PN & $\beta$ & prec. & ecc. & multi. & analy. \\
\hline Will (1994) [13] & 1.5 & $\times$ & $\times$ & $\times$ & $\times$ & PA \\
Damour \& Esposito-Farèse (1998) [14] & 1.5 & $\times$ & $\times$ & $\times$ & $\times$ & PA \\
Scharre \& Will (2002) [15] & 1.5 & $\times$ & $\times$ & $\times$ & $\times$ & PA \\
Will \& Yunes (2004) [16] & 1.5 & $\times$ & $\times$ & $\times$ & $\times$ & PA \\
Berti et al. (2005) [17] & 2 & $\checkmark$ & $\times$ & $\times$ & $\times$ & MC \\
Yagi \& Tanaka (2010) [18] & 2 & $\checkmark$ & $\checkmark$ & $\checkmark$ & $\times$ & MC \\
Yagi \& Tanaka (2011) [19] & 2 & $\checkmark$ & $\checkmark$ & $\checkmark$ & $\checkmark$ & MC \\
Arun \& Pai (2013) [20] & 2 & $\checkmark$ & $\times$ & $\times$ & $\times$ & PA \\
Yagi (2013) [21] & 2 & $\checkmark$ & $\times$ & $\times$ & $\times$ & PA \\
\hline \hline
\end{tabular}

using DECIGO/BBO are more than one order of magnitude stronger than the current bound. DECIGO/BBO performs better than LISA for three reasons. First, the number of GW cycles is larger since the GW frequency is higher, which allows one to perform more accurate measurement on parameters for a fixed SNR. Second, the velocity of binary constituents at 1yr before coalescence is smaller, which makes the "-1PN" dipolar radiation effect larger. Third, the effective frequency range is larger, which again allows one to measure parameters more accurately. DECIGO/BBO can place even stronger constraints by taking the advantage of the fact that the expected detection rate of $\mathrm{NS} / \mathrm{BH}$ binaries is $\sim 10^{4}$. Such a large detection rate makes the constraint on $\omega_{\mathrm{BD}}$ to be $\omega_{\mathrm{BD}}>3.77 \times 10^{8}$, which is indeed four orders of magnitude stronger than the solar system bound!

Recently, Arun and Pai [20] and Yagi [21] extended [17] by calculating the upper bound on $\omega_{\mathrm{BD}}$ using detectors other than LISA or DECIGO/BBO. Reference [20] found that the bound with second-generation GW interferometers, such as Adv. LIGO, is 400 times weaker than the current bound, while the bound with third generation interferometers, such as ET, can be slightly larger than the current one. Reference [21] found that the bound with ASTROD-GW is comparable to that with LISA. The bound with eLISA is also comparable to the LISA one [20,21].

3.2.2. Massive BD Theory Regarding proposed GW constraints on massive scalar-tensor theories, Berti et al. [22] derived corrections to the gravitational waveform emitted from compact binaries in massive BD theory. They showed that the quadrupolar and dipolar scalar radiation is produced when $f>\bar{m}_{s} /(2 \pi)$ and $f>\bar{m}_{s} /(\pi)$ respectively, where $\bar{m}_{s} \equiv m_{s} / \hbar$ is the reduced mass of the scalar field. The correction to the gravitational 
Table 4. Summary of the proposed bounds on $\omega_{\mathrm{BD}}$ with various GW interferometers. Numbers in brackets represent the total mass of a binary in a unit of $M_{\odot}$. This table is taken from [21].

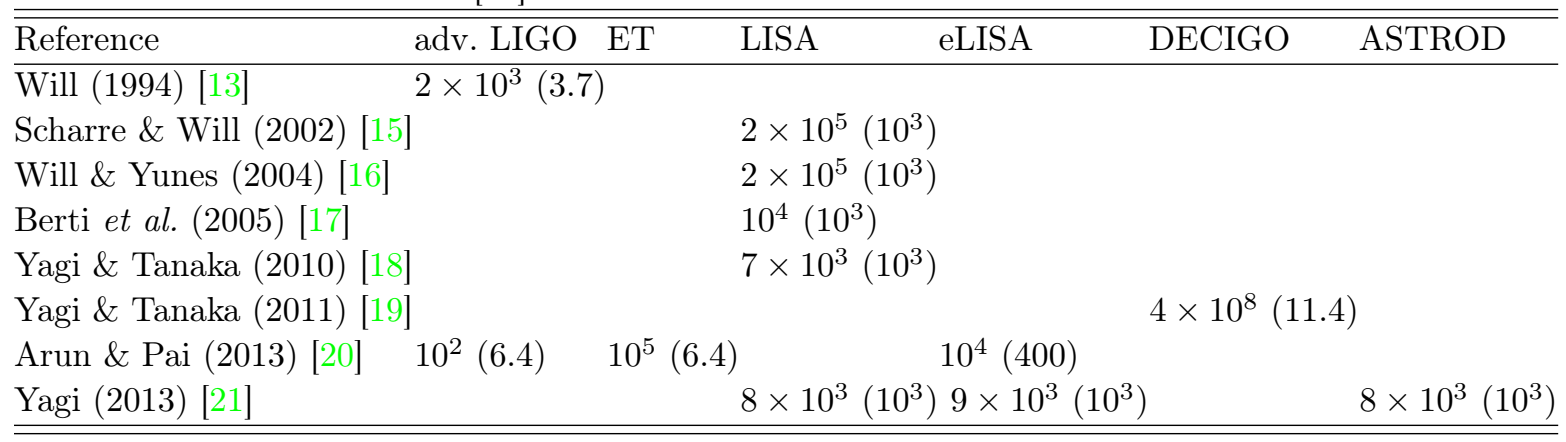

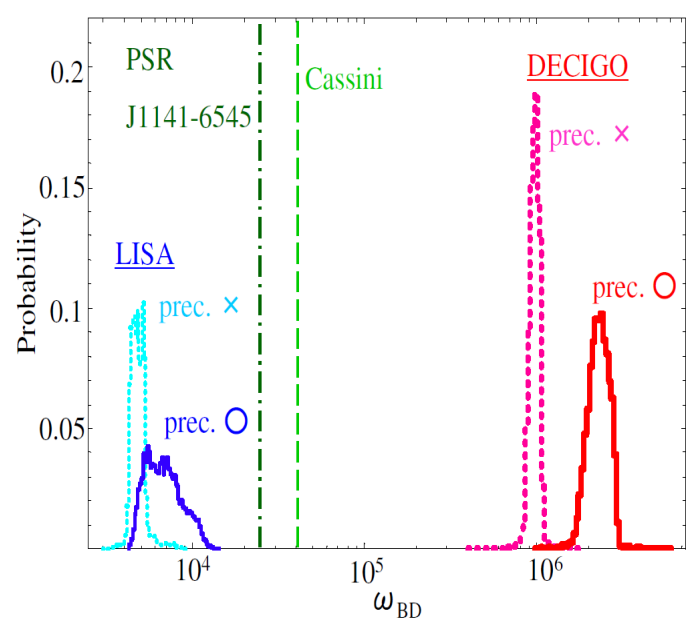

Figure 5. (Color online) Histograms showing probability distributions of the lower bound of $\omega_{\mathrm{BD}}$ obtained in $[18,19]$. The authors carried out Monte Carlo simulations of $10^{4} \mathrm{BH} / \mathrm{BH}$ binaries for a circular orbit with and without spin precessions in massless $\mathrm{BD}$ theory. The mass and distance of binaries are chosen as $\left(1.4+10^{3}\right) M_{\odot}$ for LISA and $(1.4+10) M_{\odot}$ for DECIGO/BBO, both with an SNR of $\sqrt{200}$. The light blue (magenta) dotted and blue (red) solid histograms show the probability distribution using LISA (DECIGO/BBO) without and with precessions respectively. The dashed and dotted-dashed vertical lines are the bounds from the Cassini satellite [190] and PSR J1141-6545 [185] respectively. Observe that DECIGO/BBO places a stronger constraint than the current bounds by more than an order of magnitude. Observe also that the effect of spin precession is not so significant in constraining massless BD theory. This figure is taken from [18].

waveform phase is given by [22]

$$
\begin{aligned}
\delta \Psi(f)= & \Psi_{0 \mathrm{PN}}(f)\left[\zeta+\xi \Gamma^{2} \nu\left(\frac{5}{462} x^{-3}-\frac{1}{1632} \nu x^{-6}\right) \Theta\left(2 \pi f-\bar{m}_{s}\right)\right. \\
& \left.-\xi \mathcal{S}^{2}\left(\frac{5}{84} x^{-1}-\frac{25}{1248} \nu x^{-4}\right) \Theta\left(\pi f-\bar{m}_{s}\right)\right],
\end{aligned}
$$


where $\nu \equiv \bar{m}_{s}^{2} m^{2}, \Theta$ is the Heaviside function and

$$
\begin{aligned}
\xi & \equiv \frac{1}{2+\omega_{\mathrm{BD}}}, \\
\Gamma & =1-2 \frac{s_{1} m_{1}+s_{2} m_{2}}{m}, \\
\zeta & =\frac{2}{3} \xi\left(s_{1}+s_{2}-2 s_{1} s_{2}\right)+\frac{1}{2} \xi-\frac{1}{12} \xi \Gamma^{2} \Theta\left(2 \pi f-\bar{m}_{s}\right) .
\end{aligned}
$$

They then carried out a Fisher analysis and derived proposed bounds on the BD parameter $\omega_{\mathrm{BD}}$ and $\bar{m}_{s}$ in massive BD theory with Adv. LIGO, ET and eLISA. They assumed such interferometers detect GW signals from a NS/BH binary and neglected the effect of $\mathrm{BH}$ spins. In order to have dipolar radiation present within e.g. Adv. LIGO frequency band, the scalar field mass needs to be smaller than $\mathcal{O}\left(10^{-13}\right) \mathrm{eV}$. The authors found that space-borne GW interferometers place more stringent constraints than the ground-based ones because the target frequency is lower for the former and GW frequencies produced from binaries in massive BD theory is proportional to $\bar{m}_{s}$. An eLISA measurement of GWs with an SNR of 10 can place constraints on the theory that are stronger than current bounds from Cassini and Lunar Laser Ranging experiments and binary pulsar observations.

An interesting fact regarding binary evolution in massive scalar-tensor theories was revealed in [179]. In GR, binary separation shrinks due to gravitational radiation. On the other hand, when a compact object (other than a black hole) orbits around a rotating super-massive black hole (SMBH) in massive scalar-tensor theories, orbits can float at a same separation (floating orbits) due to superradiance. Namely, if the central BH is rotating sufficiently fast, an orbiting body excites superradiant modes of the scalar field, which can fuel the orbiting body energy at the same rate as that radiated to infinity (and to the horizon of the central $\mathrm{BH}$ ) due to gravitational radiation. If the central $\mathrm{BH}$ is orbiting slowly, the scalar radiation due to superradiance that goes into the horizon becomes positive, which enhances the inspiral rate of the system (sinking orbits).

Yunes et al. [23] constructed gravitational waveform in massive BD theory for binaries with both floating and sinking orbits. Regrading the former, they found that such an effect can produce a large dephasing when carrying out a matched filtering analysis using the GR inspiral template. This means that if a GW signal from an EMRI consisting of a SMBH and a NS is detected with LISA, one can place a stringent constraint on $\omega_{\mathrm{BD}}$ for scalar field masses that produce supperradiant resonances at frequencies that can be detected with LISA. Moreover, the timescale for an orbiting body to go through the "floating" resonance becomes larger than the Hubble timescale for binaries with frequencies that are lower than the lowest frequency limit of LISA. This means that once the EMRI signal is detected, one can also rule out the parameter region that produces floating orbit at a frequency lower than the detected one. Based on these facts, the authors in [23] found that LISA can place constraints that are more than 10 orders of magnitude stronger than the current bound on the theory! Although this analysis does not consider systematic errors, it is unlikely that systematics due to e.g. astrophysical 
matter around EMRIs and uncertainties in the waveform modeling compensate such a large effect due to floating orbits. Regarding GW signals from binaries with a sinking orbit, they found that such effect is small and one cannot place meaningful constraints with LISA from such systems.

3.2.3. Quasi BD Theory Gravitational and scalar radiation from compact binaries in quasi BD theory was derived in [14]. In this theory, stars can spontaneously scalarize once their binding energy exceeds the threshold [406,407] (spontaneous scalarization). Such a phenomenon can be understood in analogy with second-order phase transitions, such as ferromagnetism. Such scalarization allows one to place stringent constraints on the theory from binary pulsar observations [4,185-187,189]. Similar scalarization [180-184] occurs in a compact binary when the separation shrinks and the binding energy of the binary exceeds the threshold (dynamical scalarization), or when a star acquires a scalar charge and scalarizes its companion (induced scalarization).

Regarding the evolution of $\mathrm{BH}$ binaries in quasi BD theory, Healy et al. [24] performed a numerical relativity simulation of late inspiral and merger of $\mathrm{BH}$ binaries in an inhomogeneous scalar field. In particular, they assumed that binaries are inside a scalar bubble. They calculated the scalar dipole radiation and found that the GWs are indistinguishable from those in GR unless an external mechanism induces dynamics in the scalar field. Alternatively, if one allows the scalar field dynamics, the scalar field bubble can collapse and accretes onto BHs, which makes the $\mathrm{BH}$ mass larger and changes the binary evolution and GWs from those in GR.

3.2.4. Generic Scalar-tensor Theories Regarding generic scalar-tensor theories, Berti et al. [25] carried out a different simulation of a $\mathrm{BH}$ binary evolution by relaxing the asymptotic flatness condition on the metric, which is motivated from cosmology [425,426]. They studied a non-spinning BH binary inspiral with a circular orbit in a constant scalar field gradient. Such a background scalar field leads to scalar dipole radiation from the binary, which can be understood from the miracle hair growth of a $\mathrm{BH}$ scalar hair found by Jacobson [408] under a time-dependent situation. Unfortunately, such scalar radiation is too small to be detected in the near future for realistic values of the scalar field gradient.

\section{3. $E M W$ Tests}

3.3.1. Quasi BD Theory Regarding EMW tests of scalar-tensor theories with a BH, Liu et al. [192] recently derived a proposed bounds on quasi BD theory from a $\mathrm{BH} /$ pulsar binary. They assumed that future radio telescopes, such as Five-hundred-metric Aperture Spherical radio Telescope (FAST) [427] and the Square Kilometre Array (SKA) [428], will detect signals from a pulsar orbiting a stellar-mass BH. They carried out a mock data simulations and estimated the measurement accuracy of post-Keplerian parameters. In particular, they applied the measurement accuracy of the orbital decay rate to constrain 
quasi BD theory. They assumed a system with the mass $(1.4,10) M_{\odot}$, the orbital period $P_{b}=5$ days, the eccentricity $e=0.8$ and the $\mathrm{BH}$ spin $\chi=0$. They found that a $5 \mathrm{yr}$ observation with FAST or SKA can place constraints that are stronger than the current bound on the coupling parameters $\alpha_{0}$ and $\beta_{0}$ in the theory. For example, the bound on $\alpha_{0}$ (which is related to the BD parameter) can be increased by more than one order of magnitude. Such a bound from BH/pulsar binaries can even be stronger than the proposed bounds from future solar system experiments, such as GAIA, in a certain parameter space of the theory.

3.3.2. Generic Scalar-tensor Theories Although a stationary BH in scalar-tensor theories does not possess a scalar hair [172], a non-stationary BH can acquire such a hair. For example, Jacobson [408] showed this perturbatively, where he assumed that the timescale in which the scalar field varies is much larger than $G M / c^{3}$ with $M$ representing the $\mathrm{BH}$ mass, and kept to the leading order in this small timescale. Such a treatment reduces to considering a time-dependent scalar field under the Kerr background. Jacobson added a term $\bar{\mu} t$ to a static scalar field under the Kerr background with $\bar{\mu}$ representing a constant. Such a term may arise from cosmological evolution or a BH slowly moving in an asymptotic spatial gradient in the scalar field. Imposing regularity at the horizon, he found that the asymptotic behavior of the scalar field around spatial infinity is given by

$$
\vartheta=\vartheta_{0}+\bar{\mu} t-\frac{2 \bar{\mu} M^{2}\left(1+\sqrt{1-\chi^{2}}\right)}{r}+\mathcal{O}\left(\frac{M^{2}}{r^{2}}\right),
$$

where $\vartheta_{0}$ is a constant that represents the scalar field at spatial infinity while $\chi \equiv J / M^{2}$ with $J$ representing the magnitude of the spin angular momentum of the BH. The coefficient of the $1 / r$ term in the above equation corresponds to the scalar hair. Notice that such a scalar field vanishes when $\bar{\mu}=0$, which clearly shows that such a scalar hair arises due to the time dependence of the scalar field. A related solution was found in shift-symmetric Horndeski theories in [416]. Horbatsch and Burgess [429] derived a constraint on $\bar{\mu}$ from the orbital decay measurement of a SMBH binary candidate OJ 287 [430]. Such a system shows a periodic burst that occurs when the smaller BH passes through the accretion disk of the larger one. They derived the dipolar radiation flux due to the emergent scalar hair above and found a bound as $\left|\bar{\mu}^{-1}\right|>1.4 \times 10^{6} \mathrm{~s}$.

\section{Massive Gravity Theories}

\subsection{Basics}

4.1.1. Theories Massive gravity theories are a simple extension of GR, where one introduces a finite mass to the graviton. Many different kinds of massive gravity theories exist (see e.g. Refs. [431-433] for recent reviews). Originally, Fierz and Pauli [193] proposed a Lorentz-invariant massive gravity model by introducing a mass term of the 
graviton in the Einstein-Hilbert action. However, such a theory shows a pathological feature that the theory does not approach GR at linear order in perturbation in the massless limit. This is known as vDVZ discontinuity [194,195] and originates from the fact that the helicity-0 component of the graviton does not decouple from matter. Although such a discontinuity seems to contradict solar system experiments, Vainshtein showed that one recovers GR in the massless limit by including the nonlinear contribution [434]. He pointed out that the linear approximation already breaks down at a distance much longer than the Schwarzschild radius (the so-called Vainshtein radius) in massive gravity. References [435,436] showed that such a mechanism indeed works in the DGP braneworld model. Although the Fierz-Pauli theory is free of ghost modes in a flat background, it suffers from the Boulware-Deser ghost in a curved background due to the presence of a helicity-0 mode of the graviton [196]. Such a pathology occurs irrespective of how one generalizes the Fierz-Pauli theory to a curved background [437].

Regarding other types of massive gravity theories, Rubakov [438] and Dubovsky [439] proposed a Lorentz violating massive gravity theory that evades pathologies related to the Bouleware-Deser instability. Although the helicity-0 mode is present in this theory, such a mode is screened thanks to the Vainshtein mechanism. Such a Lorentz-violating massive gravity theory has recently been extended so that the theory becomes UV complete by introducing vector fields [440]. Chamseddine and Mukhanov proposed a new massive gravity model inspired by the Higgs mechanism [441]. They introduced four scalar fields with a global Lorentz symmetry and when such a symmetry is spontaneously broken, the graviton absorbs scalar degrees of freedom and acquires a finite mass. Although Vainshtein mechanism seems to work also in this theory [442], the existence of ghost modes cannot be avoided [443].

Recently, de Rham et al. [197, 198] generalized the Fierz-Pauli theory in a nonlinear way and proposed nonlinear massive gravity (or ghost-free massive gravity) under a flat reference metric. Reference [198] showed that the Hamiltonian constraint, which is necessary to kill the Boulware-Deser ghost modes, exists up to fourth order in nonlinearities in the unitary gauge. Hassan and Rosen [199] generalized the theory to a generic reference metric. The Hamiltonian constraint was shown to exist in all orders in the unitary gauge with (i) flat [444], (ii) generic but non-dynamical [445], and (iii) generic and dynamical (bi-gravity) [446] reference metrics. Furthermore, Refs. [447-449] showed the existence of the secondary constraint in both nonlinear massive and bimetric gravities. This concludes that the Boulware-Deser ghost is absent to all orders in nonlinearities in these massive gravity theories. The extension of nonlinear massive gravity includes (i) mass-varying [450,451] and (ii) quasi-dilaton massive gravity [452, 453]. The former promotes the mass as a function of an external scalar field while the latter rescales the reference metric globally using the quasi-dilation scalar field so that the theory acquires a global rescaling symmetry. The absence of the Boulware-Deser ghosts in these extended theories were shown in [450,451, 454].

Regarding $\mathrm{BH}$ solutions in nonlinear massive gravity and bi-gravity, the Kerr 
spacetime is also a solution to the modified field equations in some of these theories* [456]. However, the Schwarzschild BH suffers from an instability against monopole fluctuations if the graviton mass is smaller than a threshold [457-459]. On the other hand, the Kerr BH (with two metrics differing by an overall constant) suffers from superradiant instabilities, whose time scale is orders of magnitude shorter than that for the spin-0 and spin-1 fields [458]. These studies led the authors in [460] to find numerically a new non-rotating, asymptotically flat BH solution in the theory. We refer the readers to recent reviews [461-464] for more details on BH solutions and their stability analyses in these theories.

4.1.2. Current Constraints A robust constraint on the mass of the graviton is obtained from solar system experiments. In massive gravity theories, the gravitational potential is given by a Yukawa-type form. Then, the acceleration of a planet in massive gravity theories relative to that in GR is given by

$$
\frac{a_{\mathrm{MG}}}{a_{\mathrm{GR}}}=1-\frac{1}{2}\left(\frac{r}{\lambda_{g}}\right)^{2}+\mathcal{O}\left[\left(\frac{r}{\lambda_{g}}\right)^{3}\right],
$$

where $a_{\mathrm{MG}}$ and $a_{\mathrm{GR}}$ represent the massive gravity and GR contribution to the acceleration and $\lambda_{g} \equiv h /\left(m_{g} c\right)$ is the graviton Compton length. The observation of the Kepler's third law of Mars places a constraint as [200]

$$
\lambda_{g}>2.8 \times 10^{17} \mathrm{~cm} .
$$

Other bounds on the mass of the graviton exist that are stronger than the solar system one above but are less robust due to theoretical uncertainties. Such bounds include those from bound clusters and tidal interactions between galaxies [202] and weak lensing [203]. Reference [204] derived a new bound on the mass of the graviton in nonlinear massive gravity from galactic lensing and velocity dispersion.

One can also place a bound from binary pulsar observations. For example, Ref. [201] calculated modifications to the energy flux emitted from a binary in a Fierz-Pauli type massive gravity and obtained a bound using orbital decay rate observations of PSR B1913+16 and PSR B1534+12. Such a constraint turned out to be two orders of magnitude weaker than the solar system bound. Reference [465] derived the bound on the propagation speed of the graviton in (beyond) Horndeski theories. If one assumes that such a bound is also applicable to constrain the graviton mass using Eq. (107) below, one finds $\lambda_{g} \gtrsim 3 \times 10^{15} \mathrm{~cm}$. Such a bound is comparable to the one in [201]. These current constraints are summarized in Table 5 .

\subsection{GW Tests}

* In nonlinear massive gravity, the two metrics cannot both be in a diagonal form [455]. For example, one can use the Eddington-Finkelstein coordinates instead of the Schwarzschild coordinates for finding a static and spherically symmetric solution. 
Table 5. Current bounds on $\lambda_{g}$ [31]. $h_{0}=H_{0} /(100 \mathrm{~km} / \mathrm{s} / \mathrm{Mpc})$ with $H_{0}$ representing the current Hubble constant.

\begin{tabular}{llllll}
\hline \hline & Solar system & Clusters & Weak lensing & Galaxies & Binary pulsars \\
\hline$\lambda_{g}(\mathrm{~cm})$ & $2.8 \times 10^{17}[200]$ & $6.2 \times 10^{24} h_{0}[202]$ & $1.8 \times 10^{27}[203]$ & $10^{26}[204]$ & $1.6 \times 10^{15}[201]$ \\
\hline \hline
\end{tabular}

Table 6. Summary of previous work on constraining massive gravity theories with GW observations alone. The second column shows the maximum PN order considered. (Berti et al. (2011) is left blank because they do not specify this information in the paper.) The third, fourth, fifth and sixth columns indicate whether they include the effect of the spin-orbit coupling $\beta$, higher harmonics $(\mathrm{HH})$, precession and eccentricity respectively. The seventh column presents whether they consider the inspiral phase only or all of the inspiral, merger and ringdown (IMR) phases in the evolution of a binary. The eighth column shows whether they assume a detection of GW signals from multiple sources. The ninth column describes whether they carry out a modelindependent (MI) calculation. The last column represents a type of analyses they performed (the pattern-averaged (PA), Monte Carlo (MC) or Bayesian (B).) This table is taken from [21].

\begin{tabular}{llllllllll}
\hline \hline Reference & PN & $\beta$ & HH & prec. & ecc. & IMR & multi. & MI & analy. \\
\hline \hline Will (1998) [26] & 1.5 & $\times$ & $\times$ & $\times$ & $\times$ & $\times$ & $\times$ & $\times$ & PA \\
Will \& Yunes (2004) [16] & 1.5 & $\times$ & $\times$ & $\times$ & $\times$ & $\times$ & $\times$ & $\times$ & PA \\
Berti et al. (2005) [17] & 2 & $\checkmark$ & $\times$ & $\times$ & $\times$ & $\times$ & $\times$ & $\times$ & MC \\
Arun \& Will (2009) [27] & 3.5 & $\checkmark$ & $\checkmark$ & $\times$ & $\times$ & $\times$ & $\times$ & $\times$ & PA \\
Stavridis \& Will (2009) [28] & 2 & $\checkmark$ & $\times$ & $\checkmark$ & $\times$ & $\times$ & $\times$ & $\times$ & MC \\
Yagi \& Tanaka (2010) [18] & 2 & $\checkmark$ & $\times$ & $\checkmark$ & $\checkmark$ & $\times$ & $\times$ & $\times$ & MC \\
Yagi \& Tanaka (2011) [19] & 2 & $\checkmark$ & $\times$ & $\checkmark$ & $\checkmark$ & $\times$ & $\times$ & $\times$ & MC \\
Keppel \& Ajith (2010) [29] & 3.5 & $\times$ & $\times$ & $\times$ & $\times$ & $\checkmark$ & $\times$ & $\times$ & PA \\
Del Pozzo et al. (2011) [30] & 2 & $\times$ & $\times$ & $\times$ & $\times$ & $\times$ & $\checkmark$ & $\times$ & B \\
Cornish et al. $(2011) ~[47]$ & 3.5 & $\times$ & $\times$ & $\times$ & $\times$ & $\times$ & $\times$ & $\checkmark$ & B \\
Berti et al. (2011) [31] & & $\times$ & $\times$ & $\times$ & $\times$ & $\times$ & $\checkmark$ & $\times$ & MC \\
Huwyler et al. (2011) [50] & 2 & $\checkmark$ & $\checkmark$ & $\checkmark$ & $\times$ & $\times$ & $\times$ & $\checkmark$ & MC \\
Arun \& Pai (2013) [20] & 3.5 & $\times$ & $\checkmark$ & $\times$ & $\times$ & $\times$ & $\times$ & $\times$ & PA \\
Yagi (2013) [21] & 2 & $\times$ & $\times$ & $\times$ & $\times$ & $\times$ & $\times$ & $\times$ & PA \\
\hline \hline
\end{tabular}

4.2.1. GW Signals Alone In this subsection, we review proposed bounds with future GW observations. When the mass of the graviton is non-vanishing, the group velocity of GWs is given by [26]

$$
v_{\mathrm{g}}^{2}=1-\frac{1}{f^{2} \lambda_{g}^{2}}
$$

Such a deviation from the speed of light modifies the gravitational waveform phase which can be mapped to that in the PPE waveform as [26]

$$
\beta_{\mathrm{ppE}}=-\frac{\pi^{2} D \mathcal{M}}{\lambda_{g}^{2}(1+z)}, \quad b_{\mathrm{ppE}}=-1
$$

Here, $z$ represents the source redshift and $D$ corresponds to a distance parameter defined in $[17,26]$. Notice that the above correction to the phase is of $1 P N$ relative to the leading term in GR. If the late-time acceleration of the Universe was due to some modifications 
Table 7. Summary of the proposed bounds on $\lambda_{g}$ with various GW interferometers. The bounds are normalized by $10^{18} \mathrm{~cm}$ for adv. LIGO and ET, while $10^{21} \mathrm{~cm}$ for LISA, eLISA, DECIGO and ASTROD-GW. Numbers in brackets represent the total mass of a binary in a unit of $M_{\odot}$. This table is taken from [21].

\begin{tabular}{|c|c|c|c|c|c|c|}
\hline Reference & adv. LIGO & ET & LISA & eLISA & DECIGO & ASTROD \\
\hline 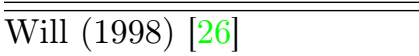 & $0.6(20)$ & & $7\left(2 \times 10^{7}\right)$ & & \multirow{14}{*}{$0.3\left(1.1 \times 10^{6}\right)$} & \\
\hline Will \& Yunes (2004) [16] & & & $5\left(10^{7}\right)$ & & & \\
\hline Berti et al. (2005) [17] & & & $1\left(2 \times 10^{6}\right)$ & & & \\
\hline Arun \& Will (2009) [27] & $0.7(60)$ & $10(400)$ & $5\left(2 \times 10^{6}\right)$ & & & \\
\hline Stavridis \& Will (2009) [28] & & & $7\left(2 \times 10^{7}\right)$ & & & \\
\hline Yagi \& Tanaka (2010) [18] & & & $3\left(1.1 \times 10^{7}\right)$ & & & \\
\hline Yagi \& Tanaka (2011) [19] & & & & & & \\
\hline Keppel \& Ajith (2010) [29] & $8(360)$ & $70(3000)$ & $60\left(4.8 \times 10^{7}\right)$ & & & \\
\hline Del Pozzo et al. (2011) [30] & $0.5-2.5$ & & & & & \\
\hline Cornish et al. (2011) [47] & $0.9(18-24)$ & & $4\left(4-5 \times 10^{6}\right)$ & & & \\
\hline Berti et al. (2011) [31] & & & $6.5-7.5$ & $3-5$ & & \\
\hline Huwyler et al. (2011) [50] & & & $7\left(1.3 \times 10^{7}\right)$ & & & \\
\hline Arun \& Pai (2013) [20] & & & & 0.1 & & \\
\hline Yagi (2013) [21] & & & $4\left(1.1 \times 10^{7}\right)$ & $1.3\left(1.1 \times 10^{7}\right)$ & & $6(1.1 \times 10$ \\
\hline
\end{tabular}

of GR that gives a mass to the graviton, one needs to require $\lambda_{g}$ to be the Hubble scale, namely $\lambda_{g} \sim 10^{28} \mathrm{~cm}$ (or the graviton mass to be $\sim 10^{-33} \mathrm{eV}$ ). We will see below that projected bounds from future GW observations with BHs are weaker than this.

Let us now briefly explain previous work on deriving proposed bounds with GW observations of $\mathrm{BH} / \mathrm{BH}$ binaries alone. We summarize the difference in computational methods and assumptions among each work in Table 6, while Table 7 shows a summary of proposed constraints with various GW interferometers with different binary systems. Will [26] derived a bound with Adv. LIGO and LISA by carrying out a Fisher analysis, where he included $\lambda_{g}$ into a parameter set that needs to be fit against the signal using the matched filtering analysis. Will and Yunes [16] carried out a similar analysis using the improved noise curve for LISA with a pattern-averaged waveform for non-spinning binaries. They studied how the constraint depends on the LISA position and acceleration noises and arm lengths. Berti et al. [17] performed Monte Carlo simulations for spinning binaries, where the authors included the spin-orbit coupling $\beta$ into the parameter set. They found a constraint $\lambda_{g}>1.33 \times 10^{21} \mathrm{~cm}$, assuming that LISA detects $\mathrm{GW}$ signals from a $\left(10^{6}+10^{6}\right) \mathrm{M}_{\odot} \mathrm{BH} / \mathrm{BH}$ binary at $3 \mathrm{Gpc}$ with an observational period of $1 \mathrm{yr}$. Observe that such a bound is roughly four orders of magnitude stronger than the solar system one.

Arun and Will [27] and Arun and Pai [20] took the effect of higher harmonics in the waveform into account and obtained a stronger bound for high-mass binaries than that obtained by using the waveform with only the dominant harmonic. Stavridis and Will [28] included the effect of spin precession by solving the precession equations numerically. They found that LISA can place $\lambda_{g}>5 \times 10^{21} \mathrm{~cm}$ by observing $\mathrm{GW}$ signals from a precessing binary with masses of $\left(10^{6}+10^{7}\right) M_{\odot}$ at $3 \mathrm{Gpc}$. Interestingly, such 
a constraint is comparable to that obtained for non-spinning binaries. This suggests that the precession breaks the degeneracy between spins and other parameters including $\lambda_{g}$. Huwyler et al. [50] included both the spin precession and higher harmonics effects. Their bound on $\lambda_{g}$ is comparable to that in [28]. Such a fact suggests that the effect of spin precession is more important than that of higher harmonics when breaking the degeneracy between $\lambda_{g}$ and other parameters.

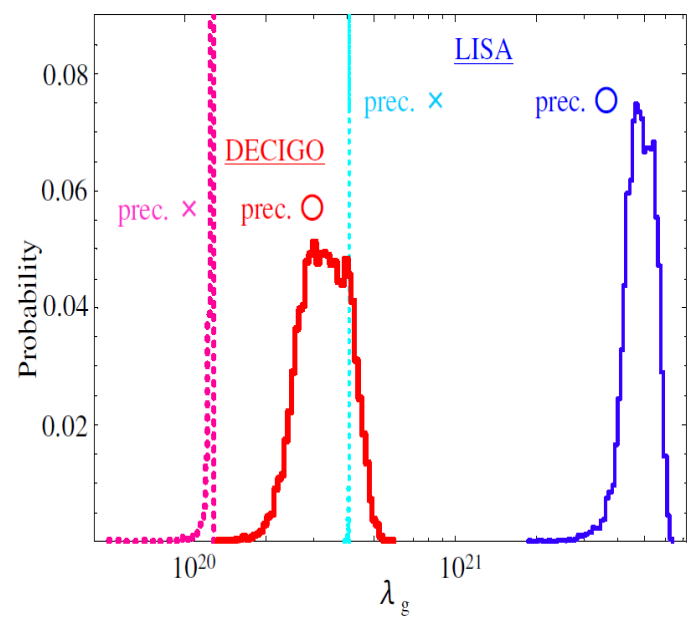

Figure 6. (Color online) Histograms showing probability distributions of the lower bound of $\lambda_{g}$ obtained in $[18,19]$. The authors carried out Monte Carlo simulations of $10^{4} \mathrm{BH} / \mathrm{BH}$ binaries for a circular orbit with and without spin precessions in massive gravity theories. The mass and distance of binaries are chosen as $\left(10^{7}+10^{6}\right) M_{\odot}$ for LISA and $\left(10^{6}+10^{5}\right) M_{\odot}$ for DECIGO/BBO, both at $3 \mathrm{Gpc}$. The meaning of each histogram is the same as in Fig. 5. Observe that LISA places a stronger constraint than DECIGO/BBO. Observe also that the precession breaks the degeneracy among parameters and improve the constraint by an order of magnitude. This figure is taken from [19].

Yagi and Tanaka $[18,19]$ included the effects of both spin precession and orbital eccentricity and derived proposed constraints on $\lambda_{g}$ with LISA and DECIGO/BBO by carrying out Monte Carlo simulations. They worked in a simple precession framework [466], where the mass of binary constituents are equal or one of the spins of the constituents is zero. One can approximately solve the precession equations analytically within such assumptions. Figure 6 presents the probability distribution of the upper bound on $\lambda_{g}$ using LISA and DECIGO/BBO with and without taking the effect of precession into account. The binary orbit is assumed to be circular. Observe that LISA places stronger constraint on $\lambda_{g}$ than DECIGO/BBO. This is because the source mass is larger for the former, which in turn brings a larger modification in the waveform phase. The histograms have a sharp peak for non-precessing binaries. This is because in such a case, $\lambda_{g}$ is completely degenerate with other parameters and the upper bound is determined purely from the prior information on spin. Observe that the spin precession enhances the constraint by an order of magnitude. This shows that the precession breaks 
the degeneracy between $\lambda_{g}$ and other parameters, consistent with [28]. Reference [18,19] found that the effect of the eccentricity is small. This is because the eccentricity and the massive gravity correction enter in the phase at negative and positive PN orders respectively, which indicates that the degeneracy between such effects are very weak. Yagi [21] estimated a bound for non-spinning binaries with eLISA and ASTROD-GW, and found that the former (the latter) bound is slightly weaker (stronger) than the LISA one.

All of the studies explained in the previous two paragraphs consider just the inspiral phase in the evolution of a binary. Keppel and Ajith [29] used the phenomenological hybrid waveform $[467,468]$ that includes not only the inspiral phase but also the merger and ringdown phases. They carried out a pattern-averaged analysis and derived bounds as $\lambda_{g}>5.9 \times 10^{22} \mathrm{~cm}$ and $\lambda_{g}>7.8 \times 10^{18} \mathrm{~cm}$ using LISA and Adv. LIGO respectively. Notice that the latter bound is a few times stronger than the solar system one. Although their analysis does not take spins of binary constituents into account, the results in Ref. [28] imply that the bounds in [29] should be comparable to those where one includes the effect of the spin precession.

Del Pozzo et al. [30] improved previous work using a Fisher analysis by performing a Bayesian inference study. They also took an advantage of the fact that future GW interferometers may detect signals from $\sim 50$ different binaries. They found a new bound of $\lambda_{g}>2.6 \times 10^{18} \mathrm{~cm}$ with second-generation GW interferometers. A similar Bayesian analysis was carried out by Cornish et al. [47] within a model-independent framework. Their constraint is consistent with that in [30]. Berti et al. [31] also took the effect of detecting GW signals from multiple sources into account. They carried out 1000 realizations of merger simulations and found that at each realization, approximately 40 BH binary mergers exist whose GW signals can be detected with LISA. They derived a bound of $\lambda_{g}>6.5-7.5 \times 10^{21} \mathrm{~cm}$ with LISA and a slightly weaker bound with eLISA.

Mirshekhari et al. [48] extended the above analyses by considering a generic modification to the dispersion relation of the graviton as

$$
E^{2}=p^{2} c^{2}+m_{g}^{2} c^{4}+A p^{\alpha} c^{\alpha},
$$

where $E$ and $p$ are the energy and linear momentum of the graviton while $A$ and $\alpha$ are constants that characterize the modification to the usual dispersion relation of the graviton. Such a modified relation includes the relation predicted in Doubly Special Relativity [469-472], extra dimension theories [473], Hořava-Lifshitz gravity [474, 475] and theories with non-commutative geometries [476]. They derived a modification to the waveform phase and found that the last term in Eq. (109) enters at $(1+3 \alpha / 2) \mathrm{PN}$ order relative to the leading. They carried out a Fisher analysis for non-spinning binaries and derived constraints on $A$ for different $\alpha$ using Adv. LIGO, ET and eLISA. For example, Adv. LIGO can typically probe the length scale of $\mathcal{O}\left(10^{-11} \mathrm{~cm}\right)$ or larger, which is much larger than the Planck scale of $\mathcal{O}\left(10^{-33} \mathrm{~cm}\right)$. Although corrections to the graviton dispersion relation are predicted to be typically Planck suppressed, this may not always be the case. For example, using perturbative quantum field theories, Collins 
et al. [477, 478] suggested that such corrections may not be Planck suppressed if one takes renormalization into account.

De Felice et al. [32] pointed out an interesting fact that gravitons of physical and reference metrics oscillate in the ghost-free bi-gravity model, just like neutrinos. Narikawa et al. [33] constructed gravitational waveforms of non-spinning compact binaries in this theory and found that a characteristic peak exists in the amplitude at a certain GW frequency that depends on the parameters in the theory. They studied the detectability of such a modified effect in gravitational waveform with second-generation ground-based detectors using a simple Bayesian hypothesis testing derived in [51]. They also carried out a Fisher analysis to study how well such interferometers can place constraints on the parameters in the theory including the effective mass of the graviton.

4.2.2. Coincident Tests with EMW Signals Let us now review proposed constraints on $\lambda_{g}$ when not only GW signals but their electromagnetic counterparts are also detected. Kocsis et al. [205] focused on a SMBH binary coalescence and proposed to use the correlation between GW and EMW signals. They derived a proposed bound of $\lambda_{g}>2.8 \times 10^{20} \mathrm{~cm}$ assuming that GWs and EMWs are emitted simultaneously and the timing uncertainty of GWs to be the inverse of the GW frequency at ISCO. Notice that such a constraint is three orders of magnitude more stringent than the solar system bound. However, such an analysis suffers from uncertainties in the delay in the emission time of GWs and EMWs, which introduce systematic errors in the bound that one can obtain. They pointed out that one can reduce such systematic errors if one can identify the variability in EMW signals before coalescence and relate that to the orbital period.

Hazboun and Larson [206] derived proposed constraints on the difference between the propagation speed of the photon and graviton by detecting EMW and GW signals simultaneously in three situations, (i) isolated pulsars with Adv. LIGO, (ii) ultracompact binaries with LISA, and (iii) supermassive BH binaries with pulsar timing arrays. The second and third situations are interesting in terms of BH based tests of GR. Although it is not clear how BH binaries can emit EMWs, such a case might be possible if a gas, such as an accretion disk, exists around them. Indeed, some known supermassive BH binaries like OJ 287 indeed show periodic variation in EMW brightness. By comparing the phase of the GW and EMW signals, they found that LISA and pulsar timing arrays may be able to place bounds on the graviton mass that is one and four orders of magnitude stronger than the solar system bound respectively.

Nishizawa and Nakamura [207] estimated how strong a coincident detection of GW and EMW signals from short gamma-ray bursts (sGRBs) can place constraints on the propagation speed of the graviton. The expected event rate of such a coincident detection with second-generation ground-based interferometers is $\sim 1 /$ yr for a NS/BH binary as the origin of a sGRB. Comparing the difference in the arrival time between gravitons and photons, they derived the bound as $\left|\delta_{g}\right|<\Delta \tau_{\text {int }} / T_{0}$, where $\delta_{g} \equiv\left(c-v_{g}\right) / c$ is the fractional difference in the propagation speed of the graviton and photon, $T_{0}$ is the propagation time duration of the photon and $\Delta \tau_{\text {int }}$ is the uncertainty in the intrinsic 
time delay between GW and EM emissions. With a typical intrinsic time delay of 10s, which is estimated from numerical calculations of long GRBs, the authors found that a coincident detection of GW and EMW signals from sGRBs can typically constrain $\delta_{g}$ to $\sim 10^{-15}$. This, in turn, leads to a constraint on $\lambda_{g}$ that is comparable to that from binary pulsar observations in [201], but weaker than the solar system bound and that from GW observations alone. Since the constraint on $\delta_{g}$ is generic, one can also apply such a bound to constrain e.g. Lorentz-violating theories of gravity such as Einstein-Æther and Hořava-Lifshitz gravity [479].

\section{Quadratic Gravity}

\subsection{Basics}

Quadratic gravity is an alternative theory of gravity where the Einstein-Hilbert action acquires a correction that depends on the Riemann curvature tensor at quadratic order coupled to a dynamical, long-ranged scalar field. Such a theory is metric in the sense that the matter field is only coupled to the metric, while the scalar field indirectly couples to matter through curvature. Matter is universally coupled to the metric, and hence, the weak equivalence principle holds.

Such a theory is motivated from at least two aspects. The first one is the bottom-up, EFT motivation, where at low energies, GR can acquire corrections that are prescribed by expanding the action in curvature. Thus, the leading correction term in the action would be at quadratic order in curvature. The second motivation is the top-down, high-energy one, where fundamental theories of quantum gravity, such as string theory [163,164] and loop quantum gravity [480,481], predict corrections to GR in which scalar fields, such as dilatons and axions, are coupled to curvature squared scalars [175, 208, 209, 482-486].

The quadratic gravity action is thus given by $[38,176]$

$$
S=S_{\mathrm{EH}}+S_{\mathrm{mat}}+S_{\vartheta}+S_{q}
$$

where

$$
S_{\mathrm{EH}}=\kappa \int d^{4} x \sqrt{-g} R
$$

is the Einstein-Hilbert action with $\kappa \equiv 1 /(16 \pi)$ while $S_{\text {mat }}$ is the action for the matter field. $S_{\vartheta}$ is the action for the canonical scalar field $\vartheta$ defined by

$$
S_{\vartheta}=-\frac{1}{2} \int d^{4} x \sqrt{-g}\left[\left(\nabla_{a} \vartheta\right)\left(\nabla^{a} \vartheta\right)+2 U(\vartheta)\right]
$$

with $U(\vartheta)$ representing the scalar field potential. $S_{q}$ contains the coupling of the scalar field to the quadratic curvature. In the EFT viewpoint, derivative interactions are higher operator order and one expects them to be suppressed relative to non-derivative interactions [214]. Restricting to non-derivative interactions only, the most generic form 
of $S_{q}$ is given by

$$
S_{q}=\int d^{4} x \sqrt{-g}\left[f_{1}(\vartheta) R^{2}+f_{2}(\vartheta) R_{a b} R^{a b}+f_{3}(\vartheta) R_{a b c d} R^{a b c d}+f_{4}(\vartheta) R_{a b c d}{ }^{*} R^{a b c d}\right],
$$

where $f_{i}(\vartheta)$ with $i=(1,2,3,4)$ are arbitrary functions of $\vartheta$ and the (left) dual Riemann tensor is defined by

$$
{ }^{*} R_{c d}^{a b}=\frac{1}{2} \epsilon^{a b e f} R_{e f c d},
$$

with $\epsilon^{a b c d}$ representing the Levi-Civita tensor.

In this review, we consider a subclass of the most generic form in Eq. (113), where we set $f_{i}(\vartheta)=\alpha_{i} g(\vartheta)$ with $\alpha_{i}$ representing coupling constants and $g(\vartheta)$ an arbitrary function of $\vartheta$, namely

$$
S_{q}=\int d^{4} x \sqrt{-g} g(\vartheta)\left[\alpha_{1} R^{2}+\alpha_{2} R_{a b} R^{a b}+\alpha_{3} R_{a b c d} R^{a b c d}+\alpha_{4}{ }^{*} R_{a b c d} R^{a b c d}\right]
$$

Notice that $\alpha_{i}$ have a unit of length squared in geometric units since $\vartheta$ is dimensionless. This subclass of quadratic gravity contains an interesting examples of theories including

- $E d G B *[208,209]: g(\vartheta)=e^{-\gamma \vartheta}$ and $\left(\alpha_{1}, \alpha_{2}, \alpha_{3}, \alpha_{4}\right)=(1,-4,1,0) \alpha_{\mathrm{EdGB}}$ with $\gamma$ and $\alpha_{\mathrm{EdGB}}$ representing coupling constants. The current strongest bound on $\alpha_{\mathrm{EdGB}}$ comes from the existence of a BH solution as $\sqrt{\left|\alpha_{\mathrm{EdGB}}\right|} \leq 1.4 \mathrm{~km}[487,488]$.

- $d C S$ [210-212]: $g(\vartheta)=\vartheta$ and $\left(\alpha_{1}, \alpha_{2}, \alpha_{3}, \alpha_{4}\right)=(0,0,0,-1 / 4) \alpha_{\mathrm{CS}}$ with $\alpha_{\mathrm{CS}}$ representing a coupling constant. The strongest bound comes from the measurement of the frame-dragging effect of Earth using Gravity Probe B and LAGEOS satellites [489] and from table-top experiments [260] as $\sqrt{\left|\alpha_{\mathrm{CS}}\right|} \leq \mathcal{O}\left(10^{8}\right) \mathrm{km}$.

- decoupled quadratic gravity (dQG) [38,176]: $g(\vartheta)=\vartheta$. Such a theory corresponds to Taylor expanding $g(\vartheta)$ in Eq. (115) around $\vartheta=0$ and keep only up to linear order in the scalar field coupling. One also neglects the term that is independent of $\vartheta$. This is motivated from a fact that such a term with either the GaussBonnet combination $\left(\left(\alpha_{1}, \alpha_{2}, \alpha_{3}, \alpha_{4}\right)=(1,-4,1,0) \alpha_{\mathrm{EdGB}}\right)$ or the CS combination $\left(\left(\alpha_{1}, \alpha_{2}, \alpha_{3}, \alpha_{4}\right)=(0,0,0,-1 / 4) \alpha_{\mathrm{CS}}\right)$ becomes a total derivative and does not contribute to the field equations. Notice that one recovers dCS gravity when one takes the CS combination of the coupling constants in dQG. The current strongest bound on $\alpha_{3}$ (or $\alpha_{\mathrm{EdGB}}$ in the decoupled EdGB) comes from the orbital decay rate of the BH LMXB A0620-00 [223] as $\sqrt{\left|\alpha_{3}\right|} \leq 1.9 \mathrm{~km}$ (or $\sqrt{\left|\alpha_{\mathrm{EdGB}}\right|} \leq 1.9 \mathrm{~km}$ ) [213].

Since we truncate the action to quadratic order in curvature, it is natural to work within the small coupling approximation, where one takes the coupling constants to be small

* EdGB theory was originally introduced in the Jordan frame [208,209], where $e^{-\gamma \vartheta}$ is coupled to the Gauss-Bonnet invariant density. If one then moves to the Einstein frame via a conformal transformation, one finds $g(\vartheta)=e^{-\gamma \vartheta}$ and $\left(\alpha_{1}, \alpha_{2}, \alpha_{3}, \alpha_{4}\right)=(1,-4,1,0) \alpha_{\text {EdGB }}$ in Eq. (115) with higher order terms in curvature tensors and (the derivative of) the scalar field. EdGB that we refer to in this review corresponds to truncated EdGB in [209] where one neglects such higher order terms. 
and keep only the leading order corrections. Such a procedure ensures that the field equations are of second order, and thus, makes the theory well-posed.* From here on, we set $U(\vartheta)=0$ for simplicity.

\subsection{BH Solutions}

In this subsection, we review static, slowly-rotating and rapidly-rotating $\mathrm{BH}$ solutions in quadratic gravity, in particular EdGB, dCS and dQG. We also review the current status of the BH stability analysis in each theory.

5.2.1. EdGB Let us first review BH solutions in EdGB gravity. Static BH solutions in EdGB were first derived in $[175,483]$ analytically within the small coupling approximation (and was later rederived in $[176,178]$ ). If one relaxes the small coupling approximation and treat the theory as exact, then one needs to solve the field equations numerically, as done in [487,491,492] for static solutions, in [488] for slowly-rotating solutions and in [484] for rapidly-rotating solutions. Reference [487] showed that the BH solutions only exist if $\alpha_{\mathrm{EdGB}}$ is smaller than a threshold that depends on the horizon radius and also the scalar field at the horizon, which places the current strongest bound on the theory.

The stability of a non-rotating BH in EdGB gravity was studied in [493] under radial perturbations, which are a special case of polar perturbations. The authors derived a Schrödinger-type master perturbation equation and solved the eigen value problem. Later, Cardoso and Pani [488] studied the stability under axial perturbations. A stability against axial perturbations is easier to tackle than that against polar perturbations because the scalar field perturbation does not appear in the axial perturbation equations. Both calculations suggest that a non-rotating $\mathrm{BH}$ is stable in EdGB gravity. Such analyses are possible because the polar and axial perturbations decouple as in GR.

5.2.2. $d C S$ We now review $\mathrm{BH}$ solutions in $\mathrm{dCS}$ gravity within the small coupling approximation. Since spherically symmetric spacetimes do not break parity, non-rotating $\mathrm{BH}$ solutions in dCS is exactly the same as the Schwarzschild solution. BH solutions acquire dCS corrections when one includes spins. Slowly rotating BH solutions to first order in spin were derived in [259,295], where the scalar field and the metric are given by

$$
\begin{aligned}
\vartheta & =\frac{5 \alpha_{\mathrm{CS}}}{8} \chi \frac{\cos \theta}{r^{2}}\left(1+\frac{2}{7} \frac{M}{r}+\frac{18}{5} \frac{M^{2}}{r^{2}}\right) \\
& =-\frac{\mu_{\mathrm{CS}}^{i} n^{i}}{r^{2}}+\mathcal{O}\left(\frac{M^{3}}{r^{3}}\right), \quad \mu_{\mathrm{CS}}^{i} \equiv-\frac{5}{8} \alpha_{\mathrm{CS}} \chi^{i}, \\
d s^{2} & =d s_{K}^{2}+\frac{5}{4} \zeta_{\mathrm{CS}} M \chi \frac{M^{4}}{r^{4}}\left(1+\frac{12}{7} \frac{M}{r}+\frac{27}{10} \frac{M^{2}}{r^{2}}\right) \sin ^{2} \theta d t d \phi .
\end{aligned}
$$

Here, $d s_{K}^{2}$ is the line element for Kerr given by Eq. (4) while $M$ and $\chi^{i}$ corresponds to the BH mass and the dimensionless spin angular momentum vector respectively. $n^{i}$ is

* This has been explicitly proven for dCS gravity in [490]. 
the unit vector from the $\mathrm{BH}$ to the field point and $\chi^{i} n_{i}=\chi \cos \theta$, while $\mu_{\mathrm{CS}}^{i}$ is the $\mathrm{BH}$ scalar dipole charge in dCS gravity. Such a scalar dipole charge is important because it sources the dominant scalar radiation emitted from a BH binary, as we will see in Sec. 5.3. $\zeta_{\mathrm{CS}}$ is the dimensionless coupling constant defined via $\zeta_{\mathrm{CS}} \equiv \alpha_{\mathrm{CS}}^{2} /\left(\kappa M^{4}\right)$. Such a modification in the metric shifts the location of ISCO.

Such calculations were extended to second order in spin in [260] using a BH perturbation scheme [494], treating a deformation due to spin as a perturbation from Kerr. The scalar field is the same as that to linear order in spin, but the metric acquires a CS correction in the even-parity sector, namely the $(t, t),(r, r),(\theta, \theta)$ and $(\phi, \phi)$ components. Such modifications to the even-parity sector render CS corrections to the quadrupole moment and the location of the event horizon, ergo region and ISCO. These modifications, in particular the one to the quadrupole moment given by $Q=Q_{K}\left[1-(201 / 1792) \zeta_{\mathrm{CS}}\right]$ with $Q_{K}$ representing the GR Kerr quadrupole moment, are crucial in modeling gravitational waveforms from BH binaries, as we will see in Sec. 5.3. Although the Kerr solution and the dCS BH solution to linear order in spin are both of Petrov type D, the dCS solution to second order in spin is of type I. This fact leads to the absence of a second-rank Killing tensor or a Carter-like constant.

Regarding rapidly rotating $\mathrm{BH}$ solutions, Ref. [39] derived the scalar dipole charge valid to all orders in spin by using the separability of the scalar wave equation, solving the $\ell=1$ mode of the scalar equation of motion, and extracting the leading (in powers of $r^{-1}$ ) behavior at spatial infinity. The authors found the magnitude of the dipole as

$$
\mu_{\mathrm{CS}}^{(\text {full })}=\alpha_{\mathrm{CS}} \frac{2+2 \chi^{4}-2 \sqrt{1-\chi^{2}}-\chi^{2}\left(3-2 \sqrt{1-\chi^{2}}\right)}{2 \chi^{3}} .
$$

Figure 7 presents the fractional difference between $\mu_{\mathrm{CS}}^{(\text {full })}$ and $\mu_{\mathrm{CS}}$. Observe that the leading order in spin charge is valid within $10 \%$ when $|\chi|<0.8$.

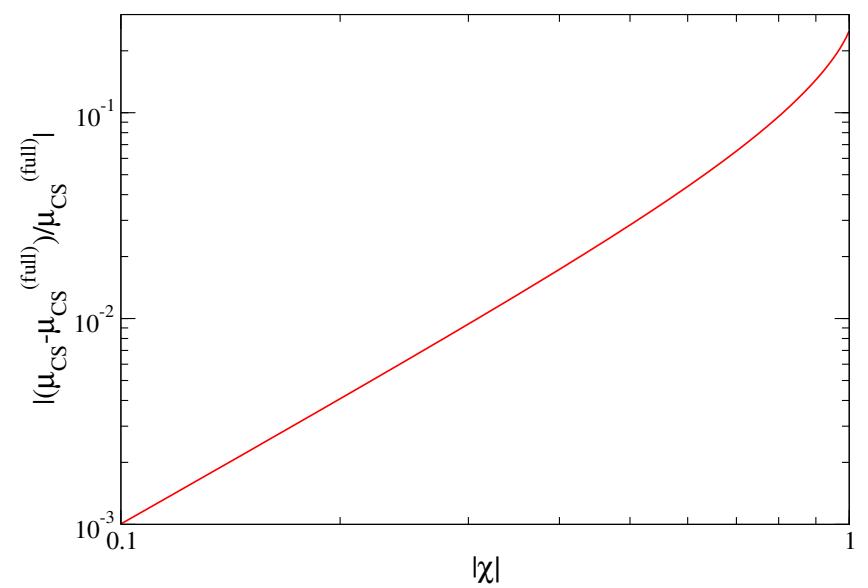

Figure 7. (Color online) Absolute fractional difference between the (magnitude of the) dipole charge to full order in spin $\mu_{\mathrm{CS}}^{(\text {full) }}$ and that to leading order in spin $\mu_{\mathrm{CS}}$ in dCS. Observe that the fractional difference is within $10 \%$ for $|\chi|<0.8$. 
In the decoupling limit, the scalar wave equation on the Kerr background separates. Each $\ell$ mode's ordinary differential equation (ODE) may be solved using variation of parameters, and the formal solution written in terms of quadrature. This has been presented in [495-497]. To be more explicit, we write

$$
\vartheta=\sum_{\ell=0}^{\infty} P_{\ell}(\cos \theta) \vartheta_{\ell}(r)
$$

in Boyer-Lindquist coordinates, where $P_{\ell}(\cos \theta)$ are Legendre polynomials. Then we find separated ODEs for each $\ell$ mode,

$$
\left[\frac{\partial}{\partial r}\left(\Delta \frac{\partial}{\partial r}\right)-\ell(\ell+1)\right] \vartheta_{\ell}(r)=S_{\ell}(r),
$$

where $S_{\ell}(r)$ was first published in [495], and then more compactly in terms of hypergeometric functions in [496]. We recall that $\Delta$ is defined below Eq. (4). Equation (120) is solved via variation of parameters using the homogeneous solutions, which are simply Legendre functions of the first and second kind, $P_{\ell}(\bar{\eta})$ and $Q_{\ell}(\bar{\eta})$, where $\bar{\eta} \equiv(r-M) / \sqrt{M^{2}-a^{2}}$ is a shifted and rescaled radial coordinate. Thus the solution for $\vartheta_{\ell}(r)$ can be written as

$$
\vartheta_{\ell}(\bar{\eta})=\vartheta_{\ell}^{+}(\bar{\eta}) P_{\ell}(\bar{\eta})+\vartheta_{\ell}^{\infty}(\bar{\eta}) Q_{\ell}(\bar{\eta}),
$$

where we have the two quadratures (due to the simplicity of the Wronskian $\mathcal{W}\left[P_{\ell}, Q_{\ell}\right]$ )

$$
\vartheta_{\ell}^{+}(\bar{\eta})=\int_{\eta}^{\infty} S_{\ell}\left(r^{\prime}\right) Q_{\ell}\left(\bar{\eta}^{\prime}\right) d \bar{\eta}^{\prime}, \quad \vartheta_{\ell}^{\infty}(\bar{\eta})=\int_{1}^{\eta} S_{\ell}\left(r^{\prime}\right) P_{\ell}\left(\bar{\eta}^{\prime}\right) d \bar{\eta}^{\prime} .
$$

Here the constants of integration are fixed by regularity at the horizon and vanishing at infinity, which means that $\vartheta_{\ell}^{+}$must vanish at infinity, while $\vartheta_{\ell}^{\infty}$ must vanish at the horizon $(\bar{\eta}=1)$.

The first few $\ell$ modes may individually be integrated in closed form (a closed-form expression for general $\ell$ has not been presented in the literature to date). However, it is straightforward to find $\vartheta_{\ell}$ numerically. Konno and Takahashi [495] integrated the radial ODEs as an initial value problem. Meanwhile, Stein [497] solved these equations using a global pseudo-spectral method. Either way, since the source is $C^{\infty}$, there is exponentially decreasing power with increasing $\ell$ number (see Fig. 8), as shown in [497]. Therefore, the solution is faithfully captured by truncating in $\ell$. At a fixed fractional truncation error, the number of required $\ell$ modes increases with increasing rotation $a$. At rapid rotation, the solution displays interesting multipolar structure as seen in Fig. 9

This solution for $\vartheta$ is the $\mathcal{O}\left(\alpha_{\mathrm{CS}}\right)$ correction to the Kerr solution. To this order, the metric is not affected. The metric is first corrected at order $\mathcal{O}\left(\alpha_{\mathrm{CS}}^{2}\right)$, as seen in Eq. (117) (recall that $\zeta_{\mathrm{CS}} \propto \alpha_{\mathrm{CS}}^{2}$ ). As mentioned before, Eq. (117) is only the $\mathcal{O}\left(a^{2}\right)$ correction to the metric. Finding the full metric to all orders in $a$ is still an open problem. However, some progress was made by Stein [497]. He noted that the trace of the metric's equation 


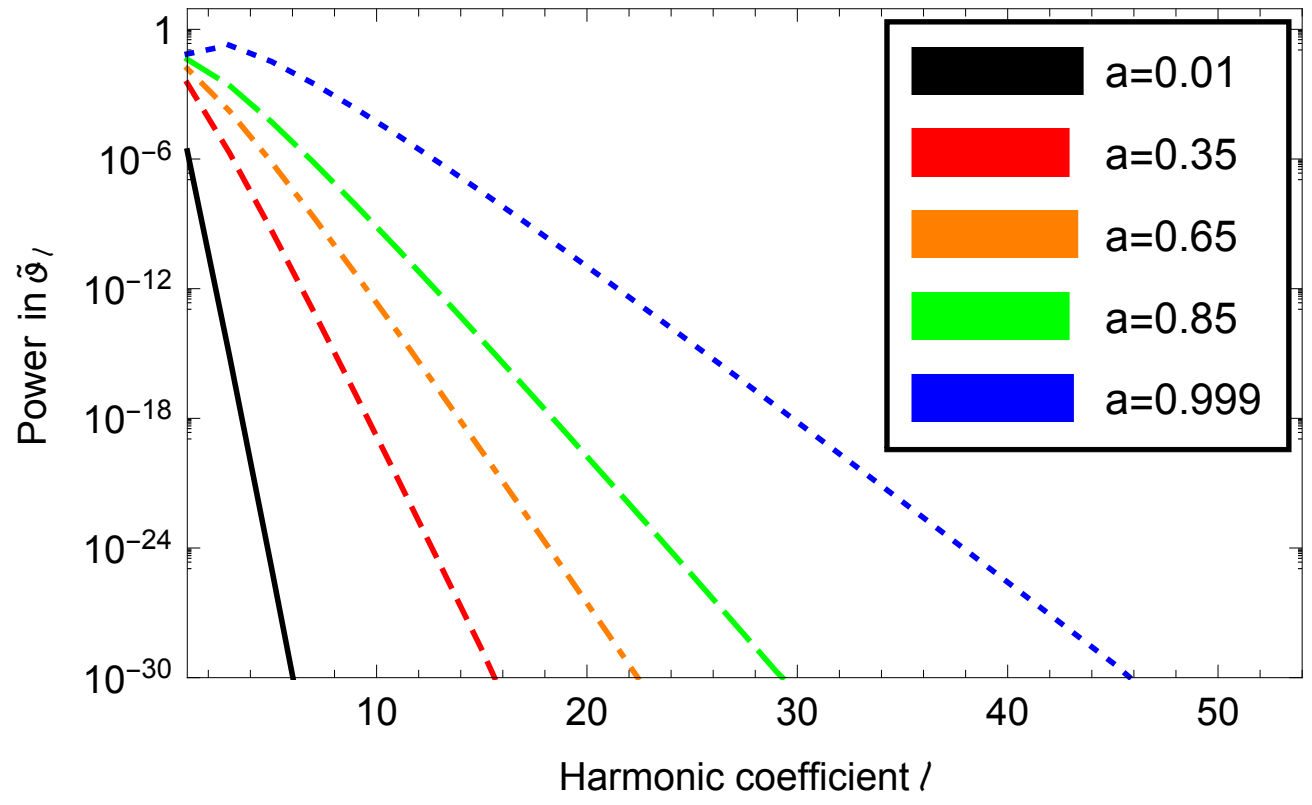

Figure 8. (Color online) Exponential convergence of smooth solutions for $\vartheta$ and $h$ as a decomposition in Legendre polynomials $P_{\ell}(\cos \theta)$. The vertical axis represents the $L^{2}$ norm of $\vartheta_{\ell}(x)$. At low spin, the convergence is more rapid, and one only needs to keep fewer coefficients, while more coefficients must be kept as one increases spin. We only plot the odd coefficients of $\vartheta . h$ follows the same trend. Figure from [497].

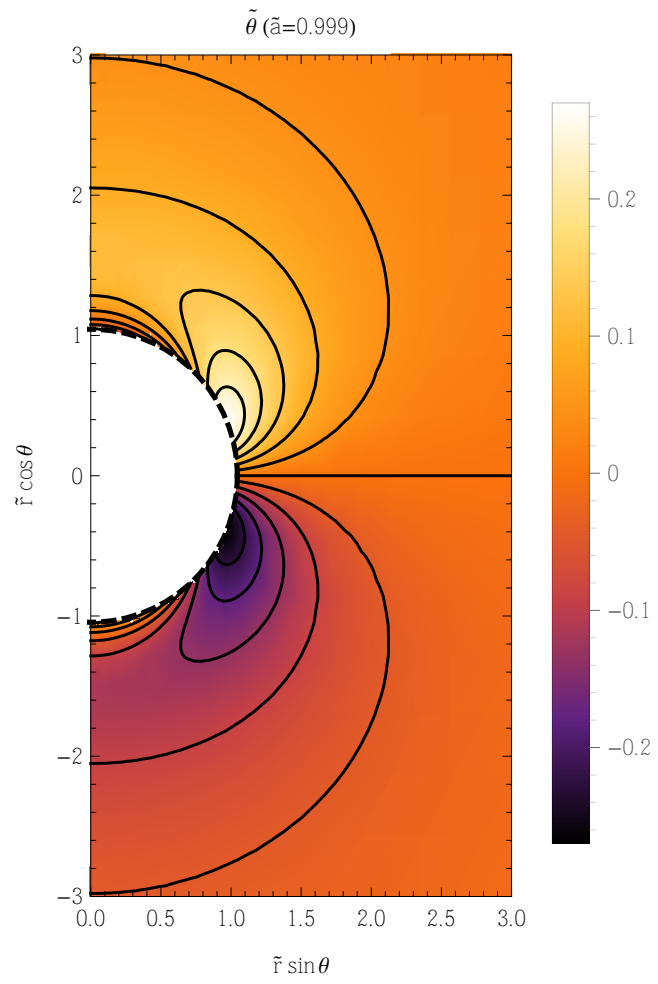

Figure 9. (Color online) Profile of solution for $\vartheta$ in a longitudinal ( $\phi=$ const.) section of the space, at high spin $(a / M=0.999)$. The rotation axis is the left edge of the figure. Color represents the value of the field and contours of constant field value are spaced linearly. The dashed line corresponds to the horizon. Figure from [497]. 
of motion, in Lorenz gauge, is simply the scalar wave operator acting on the trace of the metric deformation $h \equiv g^{a b} h_{a b}$, with a different source term:

$$
\kappa \square h=-\left(\nabla^{a} \vartheta\right)\left(\nabla_{a} \vartheta\right) .
$$

Here the source term depends on the solution for $\vartheta$ to all orders in $a$. This is the same differential operator which acts on $\vartheta$ in its equation of motion, so again separation of variables will give radial ODEs of the form of Eq. (120), acting on $h_{\ell}$ and with different source terms. This was also solved numerically in [497].

Reference [497] used this numerical solution to find the regime of validity of the weak-coupling perturbation theory. Specifically, for the perturbation theory to be valid, we should have that the size of $h$ is under perturbative control, where $h$ is the $\mathcal{O}\left(\alpha_{\mathrm{CS}}^{2}\right)$ perturbation away from the Kerr metric. If we examine the perturbation to the volume element,

$$
\sqrt{-g}=\sqrt{-g^{\mathrm{GR}}}\left(1+\frac{1}{2} h\right)+\mathcal{O}\left(\alpha_{\mathrm{CS}}^{3}\right),
$$

we see that a condition on the trace, $|h|<1$ throughout the spacetime, can serve to define the regime of validity of the perturbative scheme. The delineation between perturbation theory being valid and invalid is presented in Fig. 1 of [497].

Regarding BH stability analyses in non-dynamical CS gravity, where one sets $S_{\vartheta}=0$ in Eq. (110), Yunes and Sopuerta [498] studied the BH perturbation under the Schwarzschild background and found that, unlike in GR, polar and axial modes are generically coupled. Due to the Pontryagin constraint, generic BH oscillations are disallowed in this theory. In particular, a single parity oscillation (purely polar or axial oscillation) does not exist. The authors also looked at a stability analysis in dCS gravity, but the situation remained similar and in particular, the axial oscillations are severely suppressed. Cardoso and Gualtieri [499] showed that the polar and axial modes decouple when the background scalar field vanishes, and the scalar field perturbation only couples to the axial perturbations. Molina et al. [500] carried out detailed numerical calculations and showed that the Schwarzschild BH in dCS gravity is stable. Motohashi and Suyama [501] carried out a perturbation study under a static and spherically symmetric spacetime in both non-dynamical and dynamical CS gravity without assuming that the background scalar field vanishes. They found that the ghost modes are present unless such a background scalar field vanishes. However, the ghost modes only appear at a very high wavenumber, as shown by [502], and this wavenumber is outside of the regime of validity of dCS when treated as an EFT.

Linear stability analysis of a $\mathrm{BH}$ in dCS within the high frequency (namely, geometric optics or WKB) approximation has been carried out in [503]. The authors considered perturbations in dCS gravity and showed how the propagation speed of GWs acquires a dCS correction under the Schwarzschild background (see Sec. 5.2.3 below for a more detailed explanation of the $\mathrm{BH}$ linear stability analysis within the WKB approximation). 
5.2.3. $d Q G$ We now describe $\mathrm{BH}$ solutions in $\mathrm{dQG}$ within the small coupling approximation. Static solutions were found in [176]. The authors found that the scalar field only depends on $\alpha_{3}$ as

$$
\vartheta=\frac{2 \alpha_{3}}{M r}\left(1+\frac{M}{r}+\frac{4}{3} \frac{M^{2}}{r^{2}}\right)=\frac{\mu_{\mathrm{dQG}}}{r}+\mathcal{O}\left(\frac{M^{2}}{r^{2}}\right), \quad \mu_{\mathrm{dQG}} \equiv \frac{2 \alpha_{3}}{M},
$$

where $\mu_{\mathrm{dQG}}$ is the $\mathrm{BH}$ scalar monopole charge in $\mathrm{dQG}$. This is because within the small coupling approximation, the scalar field is source by the curvature scalar under the Kerr background and therefore $R^{2}=0=R_{\mu \nu} R^{\mu \nu}$, while $R_{a b c d}{ }^{*} R^{a b c d}=0$ due to spherically symmetry. Thus, the scalar field is only sourced by a term proportional to $\alpha_{3} R_{\mu \nu \rho \sigma} R^{\mu \nu \rho \sigma}$. The metric acquires a dQG correction to the $(t, t)$ and $(r, r)$ components, which further modifies the location of the ICSO and binding energy of a binary.

Slowly-rotating BH solutions in $\mathrm{dQG}$ were found in [504] to linear order in spin and in [505] to quadratic order in spin. As in dCS, at linear order in spin, the scalar field is proportional to $\alpha_{4}$ and the metric acquires modifications to the odd-parity sector, which further modifies the location of the ISCO. At second order in spin, the scalar field is proportional to $\alpha_{3}$ and the metric acquires modifications to the even-parity sector, which further modifies the quadrupole moment and the location of the ISCO, event horizon and ergo region. As in dCS gravity, dQG BH solutions are of Petrov type D to linear order in spin while of type I to second order in spin.

Linear stability analysis of a BH in quadratic gravity within the WKB approximation has been carried out in [506]. The authors explicitly derived the propagation speed of GWs under the slowly-rotating BH background. When the scalar field perturbation is larger than the metric one, the dispersion relation of GWs in quadratic gravity is given by [506]

$$
\left(k^{\mu} k_{\mu}\right)^{2}=32 \xi_{A} S^{\mu \nu} S_{\mu \nu}=32 \xi_{A} W^{\mu \nu} W_{\mu \nu},
$$

where $A=3(A=4)$ when considering the even (odd) parity sector of the theory, $\xi_{A} \equiv \alpha_{A} / \kappa$ and $k^{\mu}$ represents the wave vector. $S_{\mu \nu}$ and $W_{\mu \nu}$ are related to the Weyl tensor $C_{\mu \nu \rho \sigma}$ via

$$
S_{\mu \nu} \equiv C_{\mu \rho \nu \sigma} k^{\rho} k^{\sigma}, \quad W_{\mu \nu} \equiv{ }^{*} C_{\mu \rho \nu \sigma} k^{\rho} k^{\sigma}=\frac{1}{2} \epsilon_{\nu \sigma}{ }^{\alpha \beta} C_{\mu \rho \alpha \beta} k^{\rho} k^{\sigma},
$$

where $\epsilon_{\mu \nu \rho \sigma}$ is the Levi-Civita tensor. Notice that the right hand side of Eq. (126) is already proportional to $\xi_{A}$, and hence one only needs to consider the GR contribution to $S_{\mu \nu}$ and $W_{\mu \nu}$ if we only keep to leading order in the coupling constant. Observe that Eq. (126) reduces to $k^{\mu} k_{\mu}=0$ in GR, and hence GWs propagate at the speed of light irrespective of the background spacetime. From Eq. (126), one finds that the dispersion relation for GWs propagating in a radial direction under any Petrov type D spacetime reduces to that in GR.

Let us now look at the GW dispersion relation under specific example backgrounds. Parameterizing the wave vector as $k^{\mu} \equiv\left[\Omega, k_{1}, k_{2}, k_{3}\right]$, one finds the dispersion relation 
of GWs under the non-spinning BH background as $[503,506]$

$$
\Omega=\Omega_{\text {Schw }}\left[1 \pm \frac{12 M^{3}}{r^{3}} \zeta_{A}^{1 / 2}\left(1-\frac{k_{1}^{2}}{\Omega_{\text {Schw }} f^{2}}\right)\right],
$$

with the GR dispersion relation given by

$$
\Omega_{\text {Schw }} \equiv \pm \frac{1}{f} \sqrt{k_{1}^{2}+f r^{2} k_{2}^{2}+f r^{2} k_{3}^{2} \sin ^{2} \theta} .
$$

Here, $M$ is the $\mathrm{BH}$ mass, $r$ is the distance from the $\mathrm{BH}$ and $f \equiv 1-2 M / r$. Observe that the quadratic gravity correction in Eq. (128) vanishes for the spherical wavefront $\left(k_{2,3}=0\right)$ and GWs propagate at the speed of light, which is consistent with [501]. On the other hand, the quadratic gravity correction to the GW dispersion relation propagating in the radial direction does not vanish under the spinning $\mathrm{BH}$ background, but such an effect decays rapidly at spatial infinity and is quadratically proportional to the $\mathrm{BH}$ spin. This means that it is practically impossible to distinguish quadratic gravity and GR from the GW propagation observed at infinity. The situation is similar when the metric perturbation is larger than the scalar one.

\section{3. $G W$ Tests}

We now explain projected constraints on quadratic gravity with GWs from BH binaries.

5.3.1. EdGB Reference [214] recently derived an order of magnitude estimate on the future projected constraints on EdGB gravity with GWs from BH binaries. The dominant correction to the waveform is due to the scalar dipole radiation, which is proportional to the square of the difference in the scalar monopole charges of the binary constituents (see Sec. 5.3.3 for more details on how to derive scalar dipole radiation in quadratic gravity). Within the small coupling approximation, the scalar charge of a NS and a $\mathrm{BH}$ are $\mathcal{O}\left(\alpha_{\mathrm{EdGB}}^{2}\right)$ and $\mathcal{O}\left(\alpha_{\mathrm{EdGB}}\right)$ respectively. Thus, when the coupling constant is smaller than the curvature length scale of a NS or a BH in a NS/BH binary, the NS scalar charge can be neglected. With an Adv. LIGO observation of $\mathrm{SNR}=20$, Cornish et al. [47] found that the measurement accuracy of the PPE parameter $\beta_{\mathrm{ppE}}$ for the dipole radiation is $\delta \beta_{\mathrm{ppE}}=5 \times 10^{-4}$. Assuming that the $\mathrm{BH}$ scalar charge is roughly the same as that in dQG with the Gauss-Bonnet combination of $\left(\alpha_{1}, \alpha_{2}, \alpha_{3}, \alpha_{4}\right)=(1,-4,1,0) \alpha_{\mathrm{EdGB}}$, one finds an approximate $\mathrm{GW}$ projected bound from a $\mathrm{BH} / \mathrm{NS}$ binary with the mass $\left(M_{\mathrm{NS}}, M_{\mathrm{BH}}\right)=(1.4,5) M_{\odot}$ as

$$
\sqrt{\left|\alpha_{\mathrm{EdGB}}\right|} \lesssim 3.0 \mathrm{~km}\left(\frac{\delta \beta_{\mathrm{ppE}}}{5 \times 10^{-4}}\right)^{1 / 4}\left(\frac{M_{\mathrm{BH}}}{5 M_{\odot}}\right)\left(\frac{0.171}{\eta}\right)^{1 / 10},
$$

where we recall that $\eta$ is the symmetric mass ratio. Similarly, an approximate bound from a $\mathrm{BH} / \mathrm{BH}$ binary with the mass $\left(m_{1}, m_{2}\right)=(10,5) M_{\odot}$ is derived as

$$
\sqrt{\left|\alpha_{\mathrm{EdGB}}\right|} \lesssim 3.4 \mathrm{~km}\left(\frac{\delta \beta_{\mathrm{ppE}}}{5 \times 10^{-4}}\right)^{1 / 4}\left(\frac{m}{15 M_{\odot}}\right)\left(\frac{0.33}{\delta}\right)^{1 / 2}\left(\frac{\eta}{0.22}\right)^{9 / 10}
$$


where $m$ is the total mass and $\delta \equiv\left(m_{1}-m_{2}\right) / m$. Such bounds are comparable to the current strongest bound from the existence of BHs [487, 488].

5.3.2. $d C S$ GWs in theories that violate parity at the level of the field equations are known to have an amplitude birefringent effect, where the amplitude of one of the circular polarization modes is enhanced while the other one is suppressed while they propagate [507-510]. Alexander et al. [34] considered such an effect on GWs from compact binaries at a cosmological distance in (non-dynamical) CS gravity. They considered a metric perturbation around a flat Friedmann-Robertson-Walker spacetime and derived the dispersion relation for such a perturbation. They then assumed that the CS correction to the relation is small, namely, the scalar field evolves on cosmological timescales. They derived the amplitude on circularly-polarized gravitational waveform as

$$
A_{\mathrm{R}, \mathrm{L}}=A_{\mathrm{R}, \mathrm{L}}^{(\mathrm{GR})} \exp \left[\lambda_{\mathrm{R}, \mathrm{L}} \frac{k(t)}{H_{0}} \bar{\xi}(z)\right], \quad A_{\mathrm{R}, \mathrm{L}}^{(\mathrm{GR})} \equiv\left(1+\lambda_{\mathrm{R}, \mathrm{L}} \mu\right)^{2} \frac{2 \mathcal{M}}{D_{L}}\left[\frac{k(t) \mathcal{M}}{2}\right]^{2 / 3}
$$

Here, the subscript $\mathrm{R}(\mathrm{L})$ refers to the right- (left-) handed polarization, $\lambda_{\mathrm{R}}=+1$, $\lambda_{\mathrm{L}}=-1, \mathcal{M}$ is the chirp mass, $D_{L}$ represents the luminosity distance of the source, $\mu$ is the cosine angle between the observer's line-of-sight and the orbital angular momentum of the binary, $H_{0}$ is the current Hubble constant, $k(t)$ is the instantaneous wave number of the wave front that passes the GW interferometer at $t$ and $\bar{\xi}(z)$ encodes the accumulated CS correction to the amplitude due to the birefringence that depends on the source redshift $z$. From this equation, one finds

$$
\frac{A_{\mathrm{R}}}{A_{\mathrm{L}}}=\frac{1+\mu}{1-\mu} \exp \left[\frac{2 k(t) \xi(z)}{H_{0}}\right] \equiv \frac{1+\tilde{\mu}}{1-\tilde{\mu}},
$$

where $\tilde{\mu}$ is the apparent cosine angle between the line-of-sight and the orbital angular momentum that includes the CS correction. The authors in [34] carried out a Fisher analysis, assuming that the amplitude parameters are completely decoupled from the phase parameters, and found that if LISA can measure $\bar{\xi}$ to $10^{-19}$ accuracy if it detects a GW signal from an edge-on, equal-mass BH binary with $M=10^{6} M_{\odot}$ at $z=5$.

Yunes et al. [35] extended the above analysis by considering a coincident detection of a short gamma-ray burst (sGRB) and GWs from NS/NS or NS/BH binaries with second-generation ground-based detectors such as Adv. LIGO. If the GW amplitude birefringence due to parity-violation effect is present, the measured luminosity distance assuming GR is correct is different from the actual distance. They assumed that the host galaxy of the sGRB source can be identified so that one obtains its redshift. By comparing the luminosity distance estimated from such EMW observations with that obtained from GW observations, one can carry out a consistency test of gravity and constrain parity-violation effect in gravity. The authors found that one can place constraints that are typically two orders of magnitude stronger than the solar system bound from the LAGEOS satellites [211] and are comparable to the binary pulsar observations [511] in non-dynamical CS gravity. The authors also discuss that the measurement error of the 
distance from EMW observations should be subdominant as long as the source is not too close (GW SNR < 108).

GWs from EMRIs in dCS gravity was first considered by Sopuerta and Yunes [36]. They used a slowly-rotating BH solution to linear order in spin in this theory. They found that such a BH admits a Carter-like constant that is constructed from the second-rank Killing tensor. They worked within the semirelativistic approximation [512], where the trajectory of a small compact object orbiting around the massive $\mathrm{BH}$ is assumed to be a geodesic, and thus the effect of radiation reaction is neglected (though the $\mathrm{dCS}$ correction to radiation reaction can be important depending on masses and spins of binary constituents [38]). Gravitational radiation within such an approximation is calculated from the usual GR multipolar decomposition of the radiative field under the Minkowski background. The dCS correction to the gravitational waveform comes from the difference in the massive BH geometry from the Kerr one to linear order in spin. They derived a rough bound on the characteristic length scale of the theory $\xi_{\mathrm{CS}}^{1 / 4}=\sqrt{\left|\alpha_{\mathrm{CS}}\right|} / \kappa^{1 / 4}$ with future GW observations as

$$
\xi_{\mathrm{CS}}^{1 / 4}<2 \times 10^{5} \mathrm{~km}\left(\frac{\Delta}{10^{-6}}\right)\left(\frac{M}{5 \times 10^{5} M_{\odot}}\right) .
$$

Here, $M$ is the mass of the massive $\mathrm{BH}$ at the center and $\Delta$ is the accuracy to which one can distinguish the waveform phase in dCS gravity from GR that depends on the SNR and the number of GW cycles. Observe that the above proposed constraint is more than two orders of magnitude stronger than the current bound. The proposed bound becomes even stronger if one can measure GW signals from intermediate-mass ratio inspirals.

Canizares et al. [40] improved the above analysis in two ways by (i) including the radiation reaction effects and (ii) carrying out a parameter estimation study. Regarding the first extension, the authors took a hybrid approach [513], where one combines the PN approximations with the BH perturbation results [514]. They used the GR expression to take the radiation reaction effect into account, and hence, the dCS modification to the waveform again comes from the correction to the background $\mathrm{BH}$ geometry. Regarding the second extension, they carried out a Fisher analysis and found the proposed bound with LISA to be $\xi^{1 / 4}<1.4 \times 10^{4} \mathrm{~km}$, with $M=5 \times 10^{5} M_{\odot}$, which is 10 times stronger than the bound in Eq. (134).

Unlike the above analyses that focused on EMRIs with a slowly-rotating massive $\mathrm{BH}$ at the center, Pani et al. [37] considered EMRIs with non-spinning BHs. Although an isolated non-spinning BH solution in dCS gravity is the same as that in GR, GWs from non-spinning BH binaries still acquire dCS corrections from the dissipative sector (radiation reaction). The authors solved a set of three differential equations that govern the metric polar and axial perturbations and the scalar field perturbation using Green's function techniques. Using these perturbations, they found that dCS corrections to the gravitational and scalar radiation to spatial infinity are of $6 \mathrm{PN}$ and $7 \mathrm{PN}$ orders relative to the GR leading quadrupolar radiation. Interestingly, those to the horizon are larger and of $5 \mathrm{PN}$ order relative to GR. They also showed that the dCS correction to the 
number of GW cycles can exceed unity depending on the mass of the binary constituents and the CS coupling parameter. Here, the number of GW cycles exceeding unity should be take as a necessary and not sufficient condition for the dCS effect to be detected, as one needs much more cycles due to e.g. correlations between parameters and systematics.

Reference [39] derived gravitational waveforms from slowly-rotating BH binaries with comparable masses in dCS gravity. Two corrections exist; conservative and dissipative. The former arises from the correction to the quadrupole moment of a $\mathrm{BH}$ solution as discussed in Sec. 5.2.2, which enters at 2PN order relative to the leading Newtonian term in the waveform. Such a BH solution also has a scalar dipole charge as in Eq. (116), whose magnitude is given by $\mu_{\mathrm{CS}}=(5 / 8) \alpha_{\mathrm{CS}} \chi$ valid to leading order in spin [38]. This scalar dipole charge induces a dipole-dipole interaction between two BHs, which also enters at $2 \mathrm{PN}$ order in the waveform. Such an effect is similar to magnetic dipole interactions of two NSs [515]. Regarding the dissipative corrections, Ref. [38] derived the energy flux correction due to the scalar radiation that enters again at $2 \mathrm{PN}$ order. Combining all of these modifications, the authors in [39] derived corrections to gravitational waveforms from BH binaries that can easily be mapped to the PPE parameters in the gravitational waveform in Eq. (32). Carrying out a Fisher analysis, Ref. [39] found that secondgeneration ground-based GW interferometers, such as Adv. LIGO, may be able to place constraints that are six orders of magnitude stronger than the current strongest bound from solar system [489] and table-top [260] experiments.

5.3.3. $d Q G$ We now move onto GW constraints on dQG. The correction to the energy flux emitted from a BH binary was calculated in [38]. The authors perturbed the field equations around a flat background and derived the wave equations for the scalar field that is sourced by scalar charges in Eqs. (125) and (116). Solving such wave equations in the near zone, one can reproduce the BH scalar field solution in Eqs. (125) and (116) to leading order in $M / r$. One can then calculate the energy flux $\dot{E}^{(\vartheta)}$ of the scalar radiation emitted from a BH binary by solving such wave equations in the far zone.

Let us first focus on the even-parity sector, where one finds the relative scalar energy flux from gravitational energy flux in GR as [38]

$$
\dot{E}^{(\vartheta)}=\dot{E}_{\mathrm{GR}}\left(1+\frac{5}{96} \zeta_{3} \frac{\delta^{2}}{\eta^{4}} v^{-2}\right),
$$

where $\zeta_{3} \equiv \alpha_{3} /\left(\kappa m^{4}\right), v$ is the orbital velocity of the binary and we recall $\delta \equiv\left(m_{1}-m_{2}\right) / m$. Notice that such a correction is of $-1 \mathrm{PN}$ order relative to GR. One can then calculate the correction to the gravitational waveform phase, which can easily be mapped to the PPE waveform in Eq. (32) as [38]

$$
\beta_{\mathrm{ppE}}=-\frac{5}{7168} \zeta_{3} \frac{\delta^{2}}{\eta^{18 / 5}}, \quad b_{\mathrm{ppE}}=-\frac{7}{3} .
$$

Using such a waveform and based on a Bayesian analysis in [47], one obtains future projected constraints with Adv. LIGO that are the same as Eqs. (130) and (131) but 
replacing $\alpha_{\mathrm{EdGB}}$ with $\alpha_{3}[38,214]$. Approximate constraints with other GW interferometers were calculated in [213] using a Fisher analysis.

Regarding the odd parity sector, one finds that if BHs are spinning, both the scalar and gravitational radiation gives $2 \mathrm{PN}$ correction to the waveform relative to GR, as already mentioned in Sec. 5.3.2. On the other hand, if BHs are non-spinning, the corrections to the energy flux are suppressed to 7PN (scalar radiation) and 6PN (gravitational radiation) order. The scalar energy flux obtained analytically within the PN approximation in [38] for a non-spinning BH binary agrees beautifully with the numerical results in [37].

\subsection{EMW Tests}

We here review current and projected constraints on quadratic gravity with EMW observations from a system containing a $\mathrm{BH}$.

5.4.1. EdGB Regarding proposed constraints on EdGB gravity, Maselli et al. [149] calculated QPO frequencies using a slowly-rotating BH solution to linear order in spin constructed in [488]. The authors adopted the relativistic precession model [344,345] (see Sec. 2.4.4 for details). This model was applied to a BH system GRO J1655-40, where three QPOs were observed with the Rossi X-ray Timing Explorer (RXTE) [351] within $\sim 1 \%$ accuracy. In GR, such a QPO triplet can be solved for the BH mass, spin and the $\mathrm{QPO}$ radius. In order to constrain non-GR theories, one needs additional information to constrain coupling parameters in such theories. The authors in [149] assumed that future $\mathrm{X}$-ray satellite LOFT will detect two QPO triplets from the same $\mathrm{BH}$ with different QPO radii with the measurement accuracy that is 15 times higher than that with RXTE. They calculated QPO triplets for a fiducial BH in EdGB gravity with the dimensionless coupling parameter of $\zeta_{\mathrm{EdGB}}\left(\equiv \alpha_{\mathrm{EdGB}}^{2} /\left(\kappa M^{4}\right)\right)=0.1$ and recovered the $\mathrm{BH}$ mass and spin assuming that GR is the correct theory. One can constrain the theory by checking the consistency on the recovered mass and spin from each QPO triplets. They carried out a Monte Carlo simulation, together with a $\chi^{2}$-test, and found that future X-ray observations may be able to place constraints that are slightly stronger than the current theoretical bound derived from the existence of a $\mathrm{BH}$ solution.

5.4.2. $d C S$ We now review EMW tests on dCS gravity. The dCS correction to the $\mathrm{BH}$ shadows of slowly-rotating BHs to linear order in spin was studied in [131]. As shown in [36], a Carter-like constant exists for such a BH solution. Thus, null geodesic equations separate and one can follow the same procedure in GR to calculate the dCS correction to the shadows. The authors in [131] showed that such shadows are indeed affected by a non-vanishing coupling constant of the theory, but whether such an effect can be measured with future observations remains unclear, especially when one takes dCS gravity as an effective theory. The continuum X-ray spectrum from a geometrically thin, optically thick accretion disk around a slowly rotating BH to linear order in spin in 
dCS gravity was calculated in [516]. The authors showed that the dCS correction to the spectrum can clearly be seen when the dimensionless coupling parameter $\zeta_{\mathrm{CS}}\left(\equiv \xi_{\mathrm{CS}} / M^{4}\right)$ is of $\mathcal{O}(10-100)$. We note that such a large coupling constant is beyond the small coupling approximation if one wishes to treat the theory as an effective theory.

Vincent [119] improved the above analyses by taking the light bending effect into account by solving the null geodesic equations for a slowly-rotating $\mathrm{BH}$ to linear order in spin in dCS using the ray-tracing algorithm with the open-source code GYOTO [300]. The author also calculated the dCS correction to the iron line emission and QPO frequencies, with the epicyclic resonance $[517,518]$ and hot spot [519] models for the latter. The author found that the typical dCS deviation from GR in these observables is within $\sim \mathcal{O}(0.1 \%)$, which makes it extremely difficult to be measured with current or near-future observations. Moore and Gair [321] recently calculated the iron line spectrum from an accretion disk around a rotating $\mathrm{BH}$ to quadratic order in spin in dCS gravity. They carried out a Bayesian parameter estimation study and found that it would be difficult to place a meaningful constraint on the theory with future observations.

5.4.3. $d Q G$ Let us now consider how one can constrain dQG with current EMW observations. As proved in [38,214], ordinary stars such as NSs do not possess monopole scalar hair. This, in turn, means that dipolar radiation is absent from a binary system with ordinary stars. Therefore, a stringent constraint on the theory comes from a binary system where at least one of the constituents is a $\mathrm{BH}$.

One example is a BH-LMXB. Based on the scalar radiation calculation in [38], Ref. [213] derived a constraint on the theory from the upper bound on the orbital decay rate of A0620-00 [223]. If one takes the Gauss-Bonnet combination of $\left(\alpha_{1}, \alpha_{2}, \alpha_{3}, \alpha_{4}\right)=$ $(1,-4,1,0) \alpha_{\mathrm{GB}}$, the bound is obtained as $\sqrt{\left|\alpha_{\mathrm{GB}}\right|}<1.9 \mathrm{~km}$. Such a constraint is six orders of magnitude stronger than the solar system bound [520]. Although such a LMXB system has larger astrophysical uncertainties than binary pulsars, the constraint depends only weakly on such uncertainties. Furthermore, Ref. [213] showed that the unknown excess in the orbital decay rate of XTE J1118+480 [521] compared to the General-Relativity prediction can be explained by additional scalar radiation in EdGB gravity. Interestingly, such an excess cannot be explained with additional radiation in most of other alternative theories of gravity as they have already been constrained strongly from solar system experiments and binary pulsar observations.

Another example is to consider a BH/pulsar binary. Based on [192], Ref. [214] derived a proposed constraint on the theory with a $\mathrm{BH} /$ pulsar system. The authors found the future projected constraint for the Gauss-Bonnet combination of the coupling constants as

$$
\sqrt{\left|\alpha_{\mathrm{GB}}\right|}<0.065 \mathrm{~km}\left(\frac{M}{10 M_{\odot}}\right)\left(\frac{\Delta \dot{P}}{10^{-3}}\right)^{1 / 4}\left(\frac{v}{10^{-3}}\right)^{1 / 2},
$$

where $\Delta \dot{P}$ is the measurement accuracy of the orbital decay rate. Such a constraint is indeed more than one order of magnitude stronger than the LMXB bound. 


\section{Large Extra Dimension}

\subsection{Basics}

String theory predicts that our universe has more than four dimensions [163,164] with extra dimensions being compactified in a certain way. One well-known and simple example of such a compactification is the Kaluza-Klein compactification. Particle physics experiments place a strong bound on the size of the extra dimension $\ell$ as $\ell \leq 10^{-16} \mathrm{~cm}$. Arkani-Hamed et al. $[522,523]$ proposed a braneworld model (the ADD model), where the authors embedded a tension-less brane (which we live on) in a flat and compact bulk spacetime. They also assumed that ordinary matter is localized on the brane. Then, only gravitons can propagate through the bulk. The size of extra dimensions can be relatively large in the ADD model since the constraint on the gravity sector is not as strong as that on the matter sector. Moreover, the ADD model provides a novel explanation on the hierarchy problem between the Planck scale and the electroweak scale. Below, we describe in detail a different type of braneworld models proposed by Randall and Sundrum [215,524].
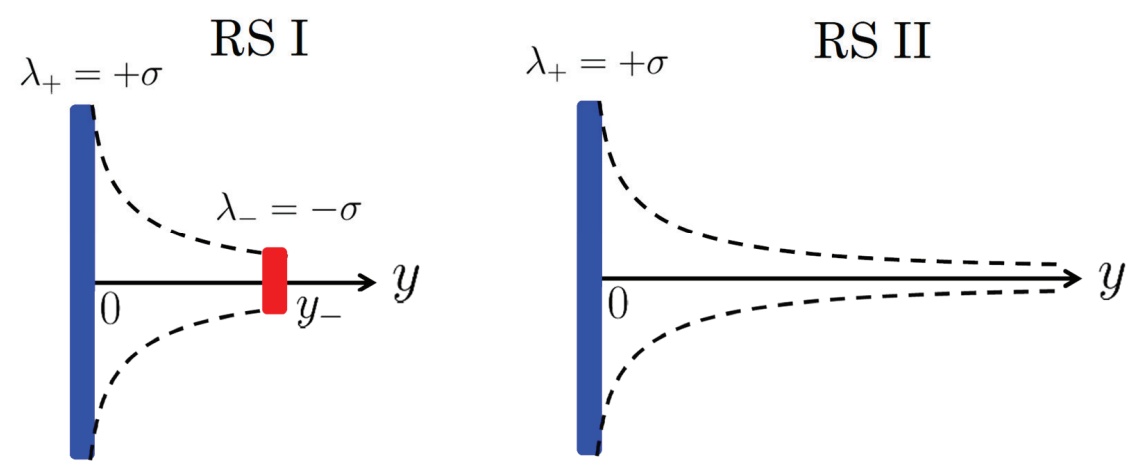

Figure 10. (Color online) Schematic picture of the RS I (left) and RS II (right) braneworld models, with $y$ representing the coordinate for the extra dimension. The bulk spacetime is AdS. The RS I model has two branes with their tension represented by $\lambda_{ \pm}$and we live on the one with a negative tension. The RS II model has one brane (with a positive tension).

6.1.1. RS braneworld Models First, we explain the first RS braneworld model (the RS I model). Let us consider a (4+1)-dimensional theory with two branes [524], one with a positive tension at $y=y_{+}=0$ and the other with a negative tension at $y=y_{-}$, where $y$ represents the coordinate of the extra dimension (see Fig. 10). We impose the $Z_{2}$ 
symmetry at $y=0$. The action is given by $*[524,525]$

$$
\begin{aligned}
S= & \frac{1}{16 \pi G_{5}} \int d^{5} x \sqrt{-g}\left(R-2 \Lambda_{5}\right) \\
& -\int d y d^{4} x \sqrt{-\gamma}\left\{\left(\lambda_{+}+L_{m+}\right) \delta(y)+\left(\lambda_{-}+L_{m-}\right) \delta\left(y-y_{-}\right)\right\} .
\end{aligned}
$$

Here, $G_{5}$ and $\Lambda_{5}$ are the five dimensional gravitational and cosmological constant respectively, $\gamma_{M N}$ represents the four dimensional induced metric on the $y=$ const. surface with the indices $(M, N)$ running from 0 to $4, \lambda_{ \pm}$are tensions and $L_{m \pm}$ are the matter Lagrangians for the positive and negative tension branes respectively. Imposing the flat brane ansatz for the metric as

$$
d s^{2}=a^{2}(y) \eta_{\mu \nu} d x^{\mu} d x^{\nu}+d y^{2}
$$

with $a(y)$ representing the warp factor, one solves the modified Einstein equations and finds

$$
a(y)=e^{-|y| / \ell}, \quad \lambda_{+}=-\lambda_{-}=\frac{3}{4 \pi G_{5} \ell} \equiv \sigma .
$$

Here, $\ell$ denotes the AdS curvature radius and is related to $\Lambda_{5}$ via $\Lambda_{5}=-6 / \ell^{2}$.

One of the interesting feature about this model is that the hierarchy problem can be explained naturally. Assuming that we live on the negative-tension brane, one finds

$$
\frac{1}{G_{4}}=\frac{\ell}{G_{5}}\left(e^{2 y_{-} / \ell}-1\right) .
$$

Therefore, by choosing $y_{-} / \ell \sim 37$, we obtain the four-dimensional Planck mass $M_{4}=10^{19} \mathrm{GeV}$ with the fundamental five-dimensional Planck mass $M_{5} \sim \ell^{-1} \sim 1 \mathrm{TeV}$. (Here, we set $\hbar=1$.)

Next, let us explain the second model [215] (the RS II model), where we take the negative tension brane to infinity. Therefore only one brane exists in this model and the size of the extra dimension is infinite (see Fig. 10). We assume that we live on the positive tension brane. Although this model cannot solve the hierarchy problem, the remarkable feature is that the Newtonian gravity is reproduced in the low energy limit even though it has an infinite size of extra dimension [216]. Perturbing the metric ansatz in Eq. (139) and solving the modified Einstein equations, one can show that all the KK modes of gravitons are suppressed and the gravity is localized on the brane. The gravitational potential is given by [215,216]

$$
V(r)=V_{\mathrm{N}}(r)\left(1+\frac{2}{3} \frac{\ell^{2}}{r^{2}}\right),
$$

where $V_{\mathrm{N}}(r)$ represents the Newtonian potential. Observe that the above equation reduces to the Newtonian potential when $\ell \ll r$. Current table-top experiments place a constraint as [217]

$$
\ell \leq 14 \mu \mathrm{m}
$$

* In this section, we do not set the gravitational constant to unity. 
6.1.2. Emparan-Fabbri-Kaloper-Tanaka Conjecture Next, we describe an important conjecture on a brane-localized black hole in RS-II braneworld model based on the AdS/CFT correspondence. Such a correspondence states that the gravity in the $A d S_{5} \times S^{5}$ spacetime can be interpreted as the four-dimensional $\mathcal{N}=4 U(N)$ super Yang-Mills theory on the AdS boundary [220,221]. This correspondence is an example of the "holographic" principle [526,527]. When such a correspondence is applied to the RS-II braneworld model [528], one finds that the five-dimensional RS-II gravity corresponds to the four-dimensional CFT on the brane interacting with the four-dimensional gravity and matter. Such a correspondence is called the bulk/brane correspondence and many supporting evidence exists as discussed in [529-536].

Regarding the CFT point of view, BHs evaporate via Hawking radiation [537]. The $\mathrm{BH}$ temperature is given by $T=\kappa_{g} / 2 \pi$ (under the unit $\hbar=1$ ) where $\kappa_{g}$ is the surface gravity of the $\mathrm{BH}$ and $\kappa_{g}=\left(4 G_{4} M\right)^{-1}$ for the Schwarzschild $\mathrm{BH}$. The radiated flux can be approximated by the black body radiation as $F \propto T^{4}$. Since the area of the $\mathrm{BH}$ event horizon is given by

$$
A^{(4 \mathrm{D})}=4 \pi\left(2 G_{4} M\right)^{2}
$$

the $\mathrm{BH}$ mass loss rate is given by $\dot{M}=F A^{(4 \mathrm{D})}=-\left(15360 \pi G_{4}^{2} M^{2}\right)^{-1}$. Therefore, in GR, the evaporation time scale becomes $\tau^{(\mathrm{GR})}=M / \dot{M}=2.1 \times 10^{67}\left(M / M_{\odot}\right)^{3} \mathrm{yr}$. On the other hand, CFT has a huge number of degrees of freedom of $N^{2} \approx \pi \ell^{2} / G_{4}=$ $2.36 \times 10^{60}(\ell / 14 \mu \mathrm{m})^{2}$ [221]. Thus, the Hawking radiation of a $\mathrm{BH}$ on a brane is enhanced compared to that in GR by a factor $\sim N^{2}$ and becomes [538]

$$
\dot{M} \propto \frac{\ell^{2}}{G_{4}^{3} M^{2}}
$$

Keeping the coefficient explicitly, one finds

$$
\dot{M}=-2.8 \times 10^{-7}\left(\frac{1 M_{\odot}}{M}\right)^{2}\left(\frac{\ell}{10 \mu \mathrm{m}}\right)^{2} M_{\odot} \mathrm{yr}^{-1}=:-C_{\dot{M}}\left(\frac{\ell}{M}\right)^{2}
$$

where $C_{\dot{M}}$ is defined as the coefficient of the mass loss rate for later use. This leads to the evaporation time scale as

$$
\tau=5.93 \times 10^{5}\left(\frac{14 \mu \mathrm{m}}{\ell}\right)^{2}\left(\frac{M}{M_{\odot}}\right)^{3} \mathrm{yr} .
$$

From the above consideration, one finds that the four-dimensional $\mathrm{BH}$ on a brane cannot remain static due to the enhanced Hawking radiation.

When we interpret such a phenomenon from the five-dimensional point of view, one finds that BHs lose their mass classically. This is the "classical BH evaporation conjecture" proposed by Emparan et al. [218] and Tanaka [219]. One possible explanation for this phenomenon in the classical picture is as follows [219,525]. Let us first consider a brane-localized BH, where the tip of such a BH is expected to form a blob having an area $\sim \ell^{3}$ with the dynamical time scale $\sim G_{4} M$ due to the Gregory-Laflamme instability [222]. 
Since a universal acceleration $\sim \ell^{-1}$ exists towards the bulk direction [539], such a blob is effectively pinched from the $\mathrm{BH}$ localized on a brane. Therefore, one obtains the relation

$$
\dot{A}^{(5 \mathrm{D})} \approx-\frac{\ell^{3}}{G_{4} M} .
$$

On the other hand, the surface area of the five-dimension $\mathrm{BH}$ is given by

$$
A^{(5 \mathrm{D})}=A^{(4 \mathrm{D})} 2 \int_{0}^{\infty} e^{-y / \ell} d y=2 A^{(4 \mathrm{D})} \ell .
$$

From Eqs. (144), (148) and (149), one finds an agreement with the mass loss rate given by Eq. (145) obtained from the four-dimensional CFT side.

\subsection{GW Tests}

In this section, we review possible future constraints with GW observations. One example is the work by Inoue and Tanaka [41], in which the authors derived the leading correction to the GW phase of compact binaries due to the correction to the gravitational potential given by Eq. (142), which is proportional to $\ell^{2} / a^{2}=\left(\ell^{2} / \mathrm{m}^{2}\right)\left(\mathrm{m}^{2} / \mathrm{a}^{2}\right)$ relative to the Newtonian term. When the Planck scale is reduced to the electroweak scale $(\sim 1 \mathrm{TeV})$, the early Universe may had a violent "mesoscopic" activity [540] when the temperature was $1 \mathrm{TeV}$ and large-amplitude fluctuations were produced, forming primordial BHs with masses $\sim 10^{-7} M_{\odot}$. The authors found a rather weak upper bound on $\ell$ assuming that third-generation $\mathrm{GW}$ interferometers detect $\mathrm{GW}$ signals from binaries with such primordial BHs. Primordial BH binaries have an advantage over astrophysical ones as the relative correction to the waveform phase becomes larger for a fixed $\ell$ and the velocity (or $m / a$ ). Below, we review other proposed constraints in detail.

We note that since static, brane-localized BH solutions have recently been numerically constructed in $[225,226]$, which contradict with the above prediction that brane-localized BHs cannot be static due to the enhanced Hawking radiation, the validity of the EmparanFabbri-Kaloper-Tanaka conjecture is now questionable. However, we proceed and present gravitational waveforms from BH binaries in RS-II model because such waveforms can easily be modified and applied to a binary with BHs losing their mass in general, such as due to phantom energy accretion* $[228,229,541]$ (one may also use GWs including the effect of gas $[107,110]$ and dark matter [103] accretion onto binary constituents). Such waveforms are also similar to those in varying $G$ theories [227].

* Assuming the Schwarzschild spacetime with a time-varying mass for the metric and a perfect fluid for matter with pressure $p$ and energy density $\rho$, one can show from the Einstein equations that the mass accretion rate is proportional to $\rho+p[228,229,541]$. Therefore, the accretion of the phantom energy with $\rho+p<0$ decreases the $\mathrm{BH}$ mass. Since it violates the weak energy condition, the theorem by Christodoulou [542] and Hawking [69] does not apply, which proves that the horizon area of a BH cannot decrease with any classical processes if the weak energy condition is satisfied. 
6.2.1. Monochromatic Signals LISA may detect GW signals that are almost monochromatic from a galactic binary composed of a $\mathrm{BH}$ and a NS with mass $m_{1}$ and $m_{2}$ respectively. Although GW emission shrinks the orbital separation $r_{12}$ (inspiral), the $\mathrm{BH}$ mass loss due to Hawking radiation increases $r_{12}$ (outspiral) at a rate of [42]

$$
\dot{r}_{12, H}=-\frac{\dot{m}_{1}}{m} a=3.2 \times 10^{-9}\left(\frac{r_{12}}{1 \mathrm{AU}}\right)\left(\frac{m}{7 M_{\odot}}\right)^{-1}\left(\frac{m_{1}}{5 M_{\odot}}\right)^{-2}\left(\frac{\ell}{14 \mu \mathrm{m}}\right)^{2} \frac{\mathrm{AU}}{\mathrm{yr}}
$$

due to the conservation of the specific orbital angular momentum $\sqrt{m r_{12}}$, where we used the mass loss rate given by Eq. (146). Equating $\dot{r}_{12, \mathrm{GW}}$ and $\dot{r}_{12, H}$ with the former calculated from the GW quadrupole formula, one finds a critical separation as [42]

$$
r_{12, \text { crit }} \equiv 1.1 \times 10^{-2}\left(\frac{m_{1}}{5 M_{\odot}}\right)^{3 / 4}\left(\frac{m_{2}}{2 M_{\odot}}\right)^{1 / 4}\left(\frac{m}{7 M_{\odot}}\right)^{1 / 2}\left(\frac{\ell}{14 \mu \mathrm{m}}\right)^{-1 / 2} \mathrm{AU} .
$$

If the separation is larger than $r_{12, \text { crit }}$, the mass loss effect dominates the GW emission and the separation becomes larger. On the other hand, if $r_{12}$ is smaller than $r_{12 \text {,crit }}$, GW emission dominates and the separation becomes smaller. Typically, a galactic BH binary forms at a GW frequency slightly outside of the LISA sensitivity band. Thus, if its signal is detected at e.g. $f=10^{-4} \mathrm{~Hz}$, one immediately finds $r_{12}\left(f=10^{-4} \mathrm{~Hz}\right) \leq r_{12 \text {,crit }}$, which then leads to [42]

$$
\ell \leq 22\left(\frac{m_{1}}{5 M_{\odot}}\right)^{3 / 2}\left(\frac{m}{7 M_{\odot}}\right)^{1 / 3}\left(\frac{m_{2}}{2 M_{\odot}}\right)^{1 / 2}\left(\frac{f}{10^{-4} \mathrm{~Hz}}\right)^{4 / 3} \mu \mathrm{m} .
$$

6.2.2. Chirping Signals Although a typical galactic BH binary forms at a GW frequency slightly lower than the low frequency limit of the LISA sensitivity band, some of them may form with a frequency higher than this lower frequency limit [543]. Therefore, a systematic error may exist when constraining the size of the extra dimension with a monochromatic GW signal. One obtains a more robust constraint by measuring the actual inspiral of a $\mathrm{BH}$ binary. Here, we first review the correction to the GW phase due to the mass loss effect derived in [43]. Then, we explain possible constraints on $\ell$ from a parameter estimation study with LISA and DECIGO/BBO. We assume that binaries are quasi-circular and neglect the spins of binary constituents for simplicity.

Let us first consider GWs from a $\mathrm{BH} / \mathrm{BH}$ binary with component masses of $m_{1}$ and $m_{2}$ (with $m_{1} \geq m_{2}$ ). The rate at which the orbital separation changes due to the mass loss effect can be derived from Eqs. (146) and (150). Then, the rate at which the GW frequency changes becomes

$$
\dot{f}=\frac{\dot{\Omega}}{\pi}=\frac{96}{5} \pi^{8 / 3} \mathcal{M}^{5 / 3} f^{11 / 3}\left(1-\frac{5}{48} C_{\dot{M}} \frac{1-2 \eta}{\eta^{3}} \frac{\ell^{2}}{m^{2}} v^{-8}\right) .
$$

From this equation, one derives the correction to the GW phase to leading order in PN approximation, which can be mapped to the PPE waveform in Eq. (32) as [43]

$$
\beta_{\mathrm{ppE}}=-\frac{25}{851968} \eta_{0}^{8 / 5} C_{\dot{M}} C \frac{\ell^{2}}{m_{0}^{2}}, \quad b_{\mathrm{ppE}}=-\frac{13}{3}
$$



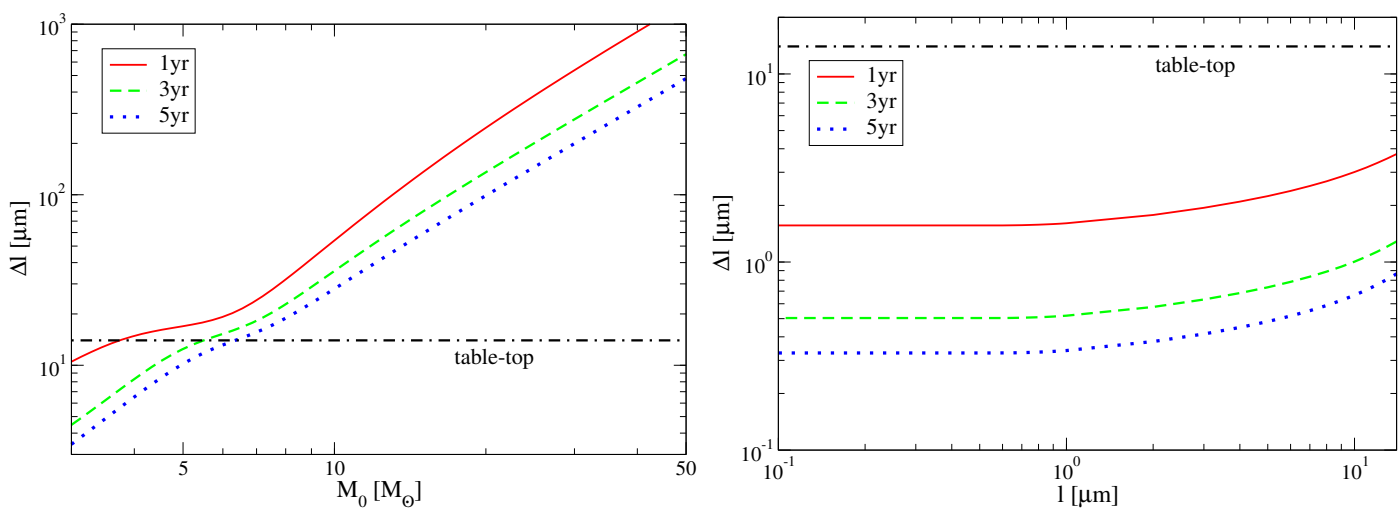

Figure 11. (Left) Proposed upper bound on $\ell$ with DECIGO/BBO from an equal mass $\mathrm{BH} / \mathrm{BH}$ binary at $D_{L}=3 \mathrm{Gpc}$ as a function of the $\mathrm{BH}$ mass $M_{0}$ with various observation period. The horizontal dotted-dashed line shows the current strongest bound from table-top experiments [217]. Observe that the bounds from GWs can be stronger than the current bound for low-mass binaries. (Right) Proposed upper bound on $\ell$ with $\mathrm{DECIGO} / \mathrm{BBO}$ from a large number of NS/BH binaries detected as a function of fiducial $\ell$. Observe that if GR is correct, the bounds from GWs can be more than 10 times stronger than the current bound as shown by the dotted-dashed line. This figure is taken and edited from [43].

where $C$ for a $\mathrm{BH} / \mathrm{BH}$ binary is given by

$$
C^{(\mathrm{BH} / \mathrm{BH})} \equiv \frac{3-26 \eta_{0}+34 \eta_{0}^{2}}{\eta_{0}^{4}}
$$

The subscript 0 represents the quantity at the time of coalescence. The second term in brackets in Eq. (154) corresponds to the " $-4 \mathrm{PN"}$ " relative correction due to the mass loss effect valid to $O\left(\ell^{2}\right)$. For a BH/NS binary, the coefficient $C$ in Eq. (155) changes to

$$
C^{(\mathrm{BH} / \mathrm{NS})}=\frac{\left(3-26 \eta_{0}+34 \eta_{0}^{2}\right)+\left(-3+20 \eta_{0}\right) \sqrt{1-4 \eta_{0}}}{2 \eta_{0}^{4}} .
$$

Notice that the GW phase in Eq. (154) is similar to that for varying $G$ theories derived in [227].

Reference [43] carried out a Fisher analysis and derived proposed bounds on $\ell$ assuming future space-borne GW interferometers detect GW chirp signals from $\mathrm{BH} / \mathrm{BH}$ or $\mathrm{BH} / \mathrm{NS}$ binaries. The authors showed that LISA can only place constraints that are 5-10 times weaker than the current bound from table-top experiments. On the other hand, DECIGO/BBO can place stronger constraints than the current bound. The left panel of Fig. 11 presents one example, where constraints on $\ell$ with DECIGO/BBO are shown as a function of the $\mathrm{BH}$ mass at the time of coalescence $M_{0}\left(=m_{1}=m_{2}\right)$ for an equal mass $\mathrm{BH} / \mathrm{BH}$ binary with various observation time. Observe that the constraints become stronger for low-mass binaries. This is because the effect of enhanced Hawking radiation becomes larger for $\mathrm{BHs}$ with smaller masses.

DECIGO/BBO can place stronger constraints on $\ell$ by taking the advantage of the fact that the expected detection rate of $\mathrm{BH} / \mathrm{NS}$ binaries is $\sim 10^{4}$. Such a detection rate 
Table 8. Proposed constraints on $\ell$ from detection rates with LISA and DECIGO/BBO. We also present other parameters used to derive these bounds. This table is taken from [43].

\begin{tabular}{lllll}
\hline \hline Detectors, binaries, masses & $\langle N\rangle$ & $r_{\mathrm{H}}$ & $\begin{array}{l}\tau \\
(\mathrm{yr})\end{array}$ & $\begin{array}{l}\ell \\
(\mu \mathrm{m})\end{array}$ \\
\hline \hline LISA, EMRI, $\left(5+10^{6}\right) M_{\odot}$ & $3.3 \times 10^{2}$ & 0.1 & $10^{9}$ & 3.9 \\
\hline DECIGO/BBO $, \mathrm{BH} / \mathrm{NS},(1.4+5) M_{\odot}$ & $9.0-13 \times 10^{4}$ & 0.1 & $6.9 \times 10^{7}$ & 15 \\
\hline \hline
\end{tabular}

depends on $\ell$, because the detection rate is reduced from that in GR if the enhanced Hawking radiation exists. The right panel of Fig. 11 presents the proposed bound $\ell$ as a function of fiducial $\ell$, taking the large number of detection events into account. The bounds are flat for small $\ell$ because in such a case, the evaporation time exceeds the age of the universe, and hence, the detection rate is the same as that in GR. Observe that if GR is correct, DECIGO/BBO can place constraints that are more than 10 times stronger than the current bound.

6.2.3. Event Rate McWilliams also derived a proposed constraint on $\ell$ from the averaged EMRI event rate $\langle\mathcal{R}\rangle_{\text {EMRI }}$. Such a rate in GR is estimated in e.g. [544]. In the RS-II braneworld model, this rate is modified due to the $\mathrm{BH}$ mass loss effect and becomes

$$
\langle\mathcal{R}\rangle_{\mathrm{H}}=r_{\mathrm{H}}\langle\mathcal{R}\rangle_{\text {EMRI }}, \quad r_{\mathrm{H}} \equiv \frac{\tau}{T_{\text {univ }}},
$$

with $\tau$ and $T_{\text {univ }}$ representing the BH lifetime given by Eq. (147) and the age of the universe respectively. The above expression is only valid for $r_{\mathrm{H}} \leq 1$. In [42], McWilliams assumed a Poisson probability distribution for the event rate and derived a constraint on $\ell$ if the GR prediction of $\langle\mathcal{R}\rangle_{\text {EMRI }}$ is actually observed.

Reference [43] extended the above analysis by taking the uncertainties in the prediction of the event rate into account. The authors in [43] assumed that due to the enhanced Hawking radiation, the detection rate $\langle N\rangle_{\mathrm{H}}$ of GWs emitted from BH binaries with future space-borne interferometers is reduced from the prediction in GR $\langle N\rangle$ by $\langle N\rangle_{\mathrm{H}}=r_{\mathrm{H}}\langle N\rangle$. If the event rate of $\langle N\rangle$ is detected as predicted in GR with an order of magnitude uncertainty, one obtains a lower bound on $r_{\mathrm{H}}$ as $r_{\mathrm{H}} \geq 0.1$. Such a bound sets a bound on $\tau$, which in turn places a bound on $\ell$ through Eq. (147). The proposed bounds on $\ell$ with LISA and DECIGO/BBO are summarized in Table 8. Observe that such proposed constraints are weaker than those from chirp GW signals mentioned in the previous subsection.

\subsection{EMW Tests}

Current astrophysical $\mathrm{BH}$ observations place constraints on $\ell$ from (i) the estimate of the $\mathrm{BH}$ mass and the age, and (ii) the measurement of the orbital decay rate of $\mathrm{BH}$ binaries. Regarding the former, Psaltis [545] derived a constraint of $\ell \leq 80 \mu \mathrm{m}$ from the estimate of the age of the BH in the X-ray binary XTE J1118+480. Gnedin et al. [546] also 
placed a constraint using an estimate of the age of the $\mathrm{BH}$ in the extra-galactic globular cluster RZ2109 as $\ell \leq 10 \mu \mathrm{m}$. Regarding the latter, Johannsen et al. [223,224] used the orbital decay rate measured in the X-ray binaries A0620-00 and XTE J1118+480 and derived constraints as $\ell \leq 161 \mu \mathrm{m}$ and $\ell \leq 970 \mu \mathrm{m}$, respectively. Recently, Simonetti et al. [547] derived a proposed, $5 \sigma$ upper bound on $\ell$ as $0.17 \mu \mathrm{m}$, assuming one measures the orbital decay rate of a BH-pulsar binary in future with the same accuracy as a 30-year observation of PSR B1913+16 [548,549].

\section{Open Questions}

We conclude this review by presenting a selected list of open questions in generic ways of testing GR and in each theory described above.

\subsection{Generic Ways of Testing GR}

- Bumpy spacetime that can describe e.g. slowly rotating BH solutions in dCS [260] and dQG [505] that are not of Petrov type D is currently lacking.

- Consistency tests of GR with parameterized PN waveform proposed in [44,45] have not been extended to spinning BHs.

- PPE waveforms [46] have not been extended to precessing binaries.

\subsection{Scalar-tensor Theories}

- The stability of hairy BH solutions in scalar-tensor theories is currently unexplored.

- Previous studies deriving proposed constraints on scalar-tensor theories with GW observations of compact binaries focus on the inspiral phase only. Since merger simulations of compact binaries in scalar-tensor theories have recently been performed [24, 25, 180-183, 550], future studies may derive new proposed bound on these theories from GW observations by including the merger and ringdown phases.

- Gravitational radiation from inspiraling compact binaries in $f(R)$ theories, which are equivalent to BD theory with a certain potential, is calculated in [551,552]. However, the sensitivities (or scalar charges) of compact objects in such theories are lacking, which are crucial for deriving proposed constraints on the theories from future GW observations.

\subsection{Massive Gravity Theories}

- Literature that derives proposed constraints on massive gravity theories with GW observations focuses mostly on how the propagation of GWs are modified. However, GW generation is also modified, which affects the gravitational waveform. Although it may be likely that the correction to the GW propagation dominates that to the GW generation, it would be important to explicitly calculate the latter effect and compare which effect dominates. 
- EMW observables for BHs in massive gravity theories remain to be calculated.

- One needs to clarify whether a linear stability analysis of BH solutions are valid in these theories, where the nonlinear Vainshtein screening mechanism plays an important role.

\subsection{Quadratic Gravity}

- BH solutions in dCS gravity have been constructed only within the slow rotation approximation. Such solutions with arbitrary rotation is currently missing.

- Stability analysis of BH solutions in quadratic gravity is incomplete. Missing pieces include polar gravitational perturbations of non-rotating BHs in EdGB gravity and any perturbations of slowly-rotating BHs to second order in spin in dQG.

- Proposed constraints on dCS gravity with GW observations derived in [39] focused on spin-aligned systems and used a Fisher analysis. Important extension of such a study includes taking spin precessions into account and carrying out a Bayesian analysis.

- EMW observables of BHs in EdGB gravity, in particular the X-ray continuum spectrum, Fe line emissions and BH shadow, remain to be studied.

- EMW observables of slowly-rotating BHs to second order in spin in dCS gravity is unexplored.

- Whether geodesic motions of a test particle around a slowly-rotating BH to quadratic order in spin in dQG and dCS become chaotic or not due to the absence of the Carter-like constant has not been studied yet.

\subsection{Other Theories}

Important non-GR theories not covered in the main text of this review include EinsteinÆther theory [151,152], which is the most general Lorentz-violating (yet diffeomorphism invariant) theory of gravity with a time-like unit vector and at most second derivatives in the action. This theory includes khronometric gravity $[553,554]$ as a certain limit, which coincides with the low-energy limit of Hořava-Lifshitz gravity [475]. Non-rotating BH solutions in Einstein-Æther theory (which are also solutions to khronometric gravity) are found in $[153,154]$, while slowly-rotating BH solutions in khronometric gravity are constructed in $[555,556]$. Barausse and Sotiriou [155] attempted to construct slowlyrotating BH solutions in Einstein-Æther theory but found that such solutions do not seem to admit universal horizons for scalar, vector and tensor propagating modes. Selected open questions regarding BHs in Einstein-Æther theory and khronometric gravity are as follows:

- Stability analysis of BH solutions in these theories are currently lacking.

- The BH sensitivities in these theories are unknown. 
- Once the BH sensitivities are calculated, one can derive current and proposed constraints on these theories with BH-LMXBs and BH-pulsar observations. One can also calculate proposed constraints from $\mathrm{BH}$ binaries with future $\mathrm{GW}$ observations.

- EMW observables of BHs in these theories remain unexplored.

\subsection{Common Open Problems}

- Theories like massive gravity and quadratic gravity in the non-decoupling limit admits various modes with different propagation speed. In such cases, multiple horizons should be present for these modes, like in Einstein-Æther theory. To the best of our knowledge, this subject has not been fully studied yet.

- More detailed studies of systematic errors in testing GR with GW and EMW observations of BHs are necessary.

\section{Acknowledgments}

We greatly thank Paolo Pani and Helvi Witek for inviting us to write this article. We also thank Enrico Barausse for carefully reading the manuscript and giving us a lot of useful comments. We further thank Alikram Aliev, Carlos Herdeiro and Nemanja Kaloper for their important feedback on the manuscript. K.Y acknowledges support from NSF CAREER Award PHY-1250636, NSF grant PHY-1305682 and JSPS Postdoctoral Fellowships for Research Abroad. L.C.S. acknowledges that support for this work was provided by the NASA through Einstein Postdoctoral Fellowship Award No. PF2130101 issued by the Chandra X-ray Observatory Center, which is operated by the Smithsonian Astrophysical Observatory for and on behalf of the National Aeronautics Space Administration under Contract No. NAS8-03060, and further acknowledges support from NSF Grant No. PHY-1068541.

\section{References}

[1] Will C M 1993 Theory and Experiment in Gravitational Physics (Cambridge University Press) ISBN 0521439736

[2] Will C M 2014 Living Rev.Rel. 174 [arXiv:1403.7377]

[3] Stairs I H 2003 Living Reviews in Relativity 6

[4] Wex N 2014 Testing Relativistic Gravity with Radio Pulsars Frontiers in Relativistic Celestial Mechanics vol 1 ed Kopeikin S (De Gruyter) ISBN 9783110345667 [arXiv:1402.5594]

[5] Murata J and Tanaka S 2015 Class.Quant.Grav. 32033001 [arXiv:1408.3588]

[6] Yagi K and Yunes N 2013 Science 341365 [arXiv:1302.4499]

[7] Yagi K and Yunes N 2013 Phys.Rev. D88 023009 [arXiv:1303.1528]

[8] Gair J R, Vallisneri M, Larson S L and Baker J G 2013 Living Rev.Rel. 167 [arXiv:1212.5575]

[9] Yunes N and Siemens X 2013 Living Rev.Rel. 169 [arXiv:1304.3473]

[10] Psaltis D 2008 Living Reviews in Relativity 11 [arXiv:0806.1531]

[11] Bambi C 2015 [arXiv:1509.03884]

[12] Psaltis D, Perrodin D, Dienes K R and Mocioiu I 2008 Phys. Rev. Lett. 100091101 [Phys. Rev. Lett.100,119902(2008)] [arXiv:0710.4564] 
[13] Will C M 1994 Phys. Rev. D50 6058-6067 [arXiv:gr-qc/9406022]

[14] Damour T and Esposito-Farese G 1998 Phys.Rev. D58 042001 [arXiv:gr-qc/9803031]

[15] Scharre P D and Will C M 2002 Phys. Rev. D65 042002 [arXiv:gr-qc/0109044]

[16] Will C M and Yunes N 2004 Class. Quant. Grav. 214367 [arXiv:gr-qc/0403100]

[17] Berti E, Buonanno A and Will C M 2005 Class. Quant. Grav. 22 S943-S954 [arXiv:gr-qc/0504017]

[18] Yagi K and Tanaka T 2010 Phys. Rev. D81 064008 [arXiv:0906.4269]

[19] Yagi K and Tanaka T 2010 Prog. Theor. Phys. 123 1069-1078 [arXiv:0908.3283]

[20] Arun K G and Pai A 2013 Int.J.Mod.Phys. D22 1341012 [arXiv:1302.2198]

[21] Yagi K 2013 Int.J.Mod.Phys. D22 1341013 [arXiv:1302.2388]

[22] Berti E, Gualtieri L, Horbatsch M and Alsing J 2012 Phys.Rev. D85 122005 [arXiv:1204.4340]

[23] Yunes N, Pani P and Cardoso V 2012 Phys.Rev. D85 102003 [arXiv:1112.3351]

[24] Healy J, Bode T, Haas R, Pazos E, Laguna P et al. 2012 Class.Quant.Grav. 29232002 [arXiv:1112.3928]

[25] Berti E, Cardoso V, Gualtieri L, Horbatsch M and Sperhake U 2013 Phys.Rev. D87 124020 [arXiv:1304.2836]

[26] Will C M 1998 Phys. Rev. D57 2061-2068 [arXiv:gr-qc/9709011]

[27] Arun K G and Will C M 2009 Class. Quant. Grav. 26155002 [arXiv:0904.1190]

[28] Stavridis A and Will C M 2009 Phys. Rev. D80 044002 [arXiv:0906.3602]

[29] Keppel D and Ajith P 2010 Phys. Rev. D82 122001 [arXiv:1004.0284]

[30] Del Pozzo W, Veitch J and Vecchio A 2011 Phys. Rev. D83 082002 [arXiv:1101.1391]

[31] Berti E, Gair J and Sesana A 2011 Phys. Rev. D84 101501 [arXiv:1107.3528]

[32] De Felice A, Nakamura T and Tanaka T 2014 PTEP 2014 043E01 [arXiv:1304.3920]

[33] Narikawa T, Ueno K, Tagoshi H, Tanaka T, Kanda N et al. 2015 Phys.Rev. D91 062007 [arXiv:1412.8074]

[34] Alexander S, Finn L S and Yunes N 2008 Phys. Rev. D78 066005 [arXiv:0712.2542]

[35] Yunes N, O'Shaughnessy R, Owen B J and Alexander S 2010 Phys.Rev. D82 064017 [arXiv:1005.3310]

[36] Sopuerta C F and Yunes N 2009 Phys.Rev. D80 064006 [arXiv:0904.4501]

[37] Pani P, Cardoso V and Gualtieri L 2011 Phys. Rev. D83 104048 [arXiv:1104.1183]

[38] Yagi K, Stein L C, Yunes N and Tanaka T 2012 Phys.Rev. D85 064022 [arXiv:1110.5950]

[39] Yagi K, Yunes N and Tanaka T 2012 Phys.Rev.Lett. 109251105 [arXiv:1208.5102]

[40] Canizares P, Gair J R and Sopuerta C F 2012 Phys.Rev. D86 044010 [arXiv:1205.1253]

[41] Inoue K T and Tanaka T 2003 Phys. Rev. Lett. 91021101 [arXiv:gr-qc/0303058]

[42] McWilliams S T 2010 Phys. Rev. Lett. 104141601 [arXiv:0912.4744]

[43] Yagi K, Tanahashi N and Tanaka T 2011 Phys. Rev. D83 084036 [arXiv:1101.4997]

[44] Arun K, Iyer B R, Qusailah M and Sathyaprakash B 2006 Class.Quant.Grav. 23 L37-L43 [arXiv:gr-qc/0604018]

[45] Arun K G, Iyer B R, Qusailah M S S and Sathyaprakash B S 2006 Phys. Rev. D74 024006 [arXiv:gr-qc/0604067]

[46] Yunes N and Pretorius F 2009 Phys.Rev. D80 122003 [arXiv:0909.3328]

[47] Cornish N, Sampson L, Yunes N and Pretorius F 2011 Phys.Rev. D84 062003 [arXiv:1105.2088]

[48] Mirshekari S, Yunes N and Will C M 2012 Phys.Rev. D85 024041 [arXiv:1110.2720]

[49] Li T, Del Pozzo W, Vitale S, Van Den Broeck C, Agathos M et al. 2012 Phys.Rev. D85 082003 [arXiv:1110.0530]

[50] Huwyler C, Klein A and Jetzer P 2012 Phys.Rev. D86 084028 [arXiv:1108.1826]

[51] Vallisneri M 2012 Phys.Rev. D86 082001 [arXiv:1207.4759]

[52] Arun K 2012 Class.Quant.Grav. 29075011 [arXiv:1202.5911]

[53] Mishra C K, Arun K G, Iyer B R and Sathyaprakash B S 2010 Phys. Rev. D82 064010 [arXiv:1005.0304]

[54] Chatziioannou K, Yunes N and Cornish N 2012 Phys.Rev. D86 022004 [arXiv:1204.2585]

[55] Vallisneri M and Yunes N 2013 Phys.Rev. D87 102002 [arXiv:1301.2627] 
[56] Sampson L, Cornish N and Yunes N 2013 Phys.Rev. D87 102001 [arXiv:1303.1185]

[57] Sampson L, Cornish N and Yunes N 2014 Phys.Rev. D89 064037 [arXiv:1311.4898]

[58] Sampson L, Yunes N and Cornish N 2013 Phys.Rev. D88 064056 [arXiv:1307.8144]

[59] Stein L C and Yagi K 2014 Phys.Rev. D89 044026 [arXiv:1310.6743]

[60] Vitale S and Del Pozzo W 2014 Phys.Rev. D89 022002 [arXiv:1311.2057]

[61] Agathos M, Del Pozzo W, Li T G F, Broeck C V D, Veitch J et al. 2014 Phys.Rev. D89 082001 [arXiv:1311.0420]

[62] Huwyler C, Porter E K and Jetzer P 2015 Phys.Rev. D91 024037 [arXiv:1410.8815]

[63] Pozzo W D, Grover K, Mandel I and Vecchio A 2014 Class.Quant.Grav. 31205006 [arXiv:1408.2356]

[64] Loutrel N, Yunes N and Pretorius F 2014 Phys.Rev. D90 104010 [arXiv:1404.0092]

[65] Israel W 1967 Phys. Rev. 164 1776-1779

[66] Israel W 1968 Commun.Math.Phys. 8 245-260

[67] Carter B 1971 Phys.Rev.Lett. 26 331-333

[68] Hawking S W 1972 Commun. Math. Phys. 25 152-166

[69] Hawking S 1971 Phys.Rev.Lett. 26 1344-1346

[70] Hansen R O 1974 Journal of Mathematical Physics 15 46-52

[71] Robinson D 1975 Phys.Rev.Lett. 34 905-906

[72] Detweiler S L 1980 Astrophys.J. 239 292-295

[73] Echeverria F 1989 Phys.Rev. D40 3194-3203

[74] Kojima Y, Yoshida S and Futamase T 1991 Prog.Theor.Phys. 86 401-410

[75] Yoshida S, Eriguchi Y and Futamase T 1994 Phys.Rev. D50 6235-6246

[76] Finn L S 1992 Phys. Rev. D46 5236-5249 [arXiv:gr-qc/9209010]

[77] Flanagan E E and Hughes S A 1998 Phys.Rev. D57 4535-4565 [arXiv:gr-qc/9701039]

[78] Dreyer O, Kelly B J, Krishnan B, Finn L S, Garrison D et al. 2004 Class.Quant.Grav. 21 787-804 [arXiv:gr-qc/0309007]

[79] Hughes S A and Menou K 2005 Astrophys.J. 623 689-699 [arXiv:astro-ph/0410148]

[80] Berti E, Cardoso V and Will C M 2006 Phys.Rev. D73 064030 [arXiv:gr-qc/0512160]

[81] Berti E and Cardoso V 2006 Int.J.Mod.Phys. D15 2209-2216 [arXiv:gr-qc/0605101]

[82] Balakrishna J, Bondarescu R, Daues G, Siddhartha Guzman F and Seidel E 2006 Class.Quant.Grav. 23 2631-2652 [arXiv:gr-qc/0602078]

[83] Berti E, Cardoso J, Cardoso V and Cavaglia M 2007 Phys.Rev. D76 104044 [arXiv:0707.1202]

[84] Chirenti C B and Rezzolla L 2007 Class.Quant.Grav. 24 4191-4206 [arXiv:0706.1513]

[85] Pani P, Berti E, Cardoso V, Chen Y and Norte R 2009 Phys.Rev. D80 124047 [arXiv:0909.0287]

[86] Kamaretsos I, Hannam M, Husa S and Sathyaprakash B 2012 Phys.Rev. D85 024018 [arXiv:1107.0854]

[87] Gossan S, Veitch J and Sathyaprakash B 2012 Phys.Rev. D85 124056 [arXiv:1111.5819]

[88] Macedo C F, Pani P, Cardoso V and Crispino L C B 2013 Phys.Rev. D88 064046 [arXiv:1307.4812]

[89] Meidam J, Agathos M, Van Den Broeck C, Veitch J and Sathyaprakash B 2014 Phys.Rev. D90 064009 [arXiv:1406.3201]

[90] Nakano H, Tanaka T and Nakamura T 2015 Phys. Rev. D92 064003 [arXiv:1506.00560]

[91] Ryan F D 1995 Phys. Rev. D52 5707-5718

[92] Ryan F D 1997 Phys. Rev. D56 1845-1855

[93] Kesden M, Gair J and Kamionkowski M 2005 Phys.Rev. D71 044015 [arXiv:astro-ph/0411478]

[94] Barack L and Cutler C 2007 Phys. Rev. D75 042003 [arXiv:gr-qc/0612029]

[95] Glampedakis K and Babak S 2006 Class. Quant. Grav. 23 4167-4188 [arXiv:gr-qc/0510057]

[96] Gair J R, Li C and Mandel I 2008 Phys.Rev. D77 024035 [arXiv:0708.0628]

[97] Apostolatos T A, Lukes-Gerakopoulos G and Contopoulos G 2009 Phys.Rev.Lett. 103111101 [arXiv:0906.0093]

[98] Lukes-Gerakopoulos G, Apostolatos T A and Contopoulos G 2010 Phys.Rev. D81 124005 [arXiv:1003.3120] 
[99] Contopoulos G, Lukes-Gerakopoulos G and Apostolatos T 2011 Int.J.Bifurc. Chaos 21 2261-2277 [arXiv:1108.5057]

[100] Pani P, Berti E, Cardoso V, Chen Y and Norte R 2010 Phys.Rev. D81 084011 [arXiv:1001.3031] [101] Gair J and Yunes N 2011 Phys.Rev. D84 064016 [arXiv:1106.6313]

[102] Wade M, Creighton J D E, Ochsner E and Nielsen A B 2013 Phys.Rev. D88 083002 [arXiv:1306.3901]

[103] Macedo C F, Pani P, Cardoso V and Crispino L C 2013 Astrophys.J. 77448 [arXiv:1302.2646]

[104] Barausse E, Cardoso V and Pani P 2014 Phys. Rev. D89 104059 [arXiv:1404.7149]

[105] Barausse E, Cardoso V and Pani P 2015 J. Phys. Conf. Ser. 610012044 [arXiv:1404.7140]

[106] Narayan R 2000 Astrophys. J. 536 663-667 [arXiv:arXiv:astro-ph/9907328]

[107] Barausse E and Rezzolla L 2008 Phys. Rev. D77 104027 [arXiv:0711.4558]

[108] Yunes N, Coleman Miller M and Thornburg J 2011 Phys. Rev. D83 044030 [arXiv:1010.1721]

[109] Yunes N, Kocsis B, Loeb A and Haiman Z 2011 Phys. Rev. Lett. 107171103 [arXiv:1103.4609]

[110] Kocsis B, Yunes N and Loeb A 2011 Phys. Rev. D84 024032 [arXiv:1104.2322]

[111] Hayasaki K, Yagi K, Tanaka T and Mineshige S 2013 Phys. Rev. D87 044051 [arXiv:1201.2858]

[112] Barack L 2009 Class. Quant. Grav. 26213001 [arXiv:0908.1664]

[113] Poisson E, Pound A and Vega I 2011 Living Rev. Rel. 147 [arXiv:1102.0529]

[114] Harko T, Kovacs Z and Lobo F S 2009 Phys.Rev. D80 044021 [arXiv:0907.1449]

[115] Harko T, Kovacs Z and Lobo F S 2009 Class.Quant.Grav. 26215006 [arXiv:0905.1355]

[116] Harko T, Kovacs Z and Lobo F S 2011 Class.Quant.Grav. 28165001 [arXiv:1009.1958]

[117] Bambi C and Barausse E 2011 Astrophys.J. 731121 [arXiv:1012.2007]

[118] Bambi C 2013 JCAP 1308 055 [arXiv:1305.5409]

[119] Vincent F 2013 Class.Quant.Grav. 31025010 [arXiv:1311.3251]

[120] Johannsen T 2014 Phys.Rev. D90 064002 [arXiv:1501.02815]

[121] Kong L, Li Z and Bambi C 2014 Astrophys.J. 79778 [arXiv:1405.1508]

[122] Bambi C 2014 Phys.Rev. D90 047503 [arXiv:1408.0690]

[123] Johannsen T and Psaltis D 2013 Astrophys.J. 77357 [arXiv:1202.6069]

[124] Bambi C 2013 Phys.Rev. D87 023007 [arXiv:1211.2513]

[125] Bambi C and Malafarina D 2013 Phys.Rev. D88 064022 [arXiv:1307.2106]

[126] Bambi C 2013 Phys.Rev. D87 084039 [arXiv:1303.0624]

[127] Jiang J, Bambi C and Steiner J F 2015 JCAP 1505025 [arXiv:1406.5677]

[128] Jiang J, Bambi C and Steiner J F 2015 Astrophys. J. 811130 [arXiv:1504.01970]

[129] Broderick A E and Loeb A 2006 Astrophys.J. 636 L109-L112 [arXiv:astro-ph/0508386]

[130] Johannsen T and Psaltis D 2010 Astrophys.J. 718 446-454 [arXiv:1005.1931]

[131] Amarilla L, Eiroa E F and Giribet G 2010 Phys.Rev. D81 124045 [arXiv:1005.0607]

[132] Amarilla L and Eiroa E F 2012 Phys.Rev. D85 064019 [arXiv:1112.6349]

[133] Bambi C, Caravelli F and Modesto L 2012 Phys.Lett. B711 10-14 [arXiv:1110.2768]

[134] Amarilla L and Eiroa E F 2013 Phys.Rev. D87 044057 [arXiv:1301.0532]

[135] Li Z and Bambi C 2014 JCAP 1401041 [arXiv:1309.1606]

[136] Broderick A E, Johannsen T, Loeb A and Psaltis D (Perimeter Institute for Theoretical Physics) 2014 Astrophys.J. 7847 [arXiv:1311.5564]

[137] Atamurotov F, Abdujabbarov A and Ahmedov B 2013 Astrophys.Space Sci. 348 179-188

[138] Wei S W and Liu Y X 2013 JCAP 1311063 [arXiv:1311.4251]

[139] Atamurotov F, Abdujabbarov A and Ahmedov B 2013 Phys.Rev. D88 064004

[140] Johannsen T 2013 Astrophys.J. 777170 [arXiv:1501.02814]

[141] Tsukamoto N, Li Z and Bambi C 2014 JCAP 1406043 [arXiv:1403.0371]

[142] Bambi C 2015 Class.Quant.Grav. 32065005 [arXiv:1409.0310]

[143] Psaltis D, Ozel F, Chan C K and Marrone D P 2015 Astrophys. J. 814115 [arXiv:1411.1454]

[144] Wei S W, Cheng P, Zhong Y and Zhou X N 2015 JCAP 1508004 [arXiv:1501.06298]

[145] Moffat J 2015 Eur.Phys.J. C75 130 [arXiv:1502.01677]

[146] Johannsen T and Psaltis D 2011 Astrophys.J. 72611 [arXiv:1010.1000] 
[147] Bambi C 2012 JCAP 1209014 [arXiv:1205.6348]

[148] Bambi C 2015 Eur.Phys.J. C75 162 [arXiv:1312.2228]

[149] Maselli A, Gualtieri L, Pani P, Stella L and Ferrari V 2015 Astrophys.J. 801115 [arXiv:1412.3473]

[150] Barausse E and Sotiriou T P 2008 Phys. Rev. Lett. 101099001 [arXiv:0803.3433]

[151] Jacobson T and Mattingly D 2001 Phys.Rev. D64 024028 [arXiv:gr-qc/0007031]

[152] Jacobson T 2007 PoS QG-PH 020 [arXiv:0801.1547]

[153] Eling C and Jacobson T 2006 Class.Quant.Grav. 23 5643-5660 [arXiv:gr-qc/0604088]

[154] Barausse E, Jacobson T and Sotiriou T P 2011 Phys.Rev. D83 124043 [arXiv:1104.2889]

[155] Barausse E and Sotiriou T P 2013 Class.Quant.Grav. 30244010 [arXiv:1307.3359]

[156] Arvanitaki A, Dimopoulos S, Dubovsky S, Kaloper N and March-Russell J 2010 Phys. Rev. D81 123530 [arXiv:0905.4720]

[157] Arvanitaki A and Dubovsky S 2011 Phys. Rev. D83 044026 [arXiv:1004.3558]

[158] Yoshino H and Kodama H 2012 Prog. Theor. Phys. 128 153-190 [arXiv:1203.5070]

[159] Yoshino H and Kodama H 2014 PTEP 2014 043E02 [arXiv:1312.2326]

[160] Yoshino H and Kodama H 2014 PTEP 2015 061E01 [arXiv:1407.2030]

[161] Arvanitaki A, Baryakhtar M and Huang X 2015 Phys. Rev. D91 084011 [arXiv:1411.2263]

[162] Fujii Y and Maeda K I 2003 The Scalar-Tensor Theory of Gravitation (Cambridge University Press) ISBN 0521811597

[163] Polchinski J 2007 String theory. Vol. 1: An introduction to the bosonic string (Cambridge University Press) ISBN 9780511252273, 9780521672276, 9780521633031

[164] Polchinski J 2007 String theory. Vol. 2: Superstring theory and beyond (Cambridge University Press) ISBN 9780511252280, 9780521633048, 9780521672283

[165] Perrotta F, Baccigalupi C and Matarrese S 2000 Phys. Rev. D61 023507 [arXiv:astro-ph/9906066]

[166] Steinhardt P J and Accetta F S 1990 Phys. Rev. Lett. 642740

[167] Hawking S W 1972 Comm. Math. Phys. 25 167-171

[168] Thorne K S and Dykla J J 1971 Astrophys. J. Lett. 166 L35

[169] Chase J E 1970 Communications in Mathematical Physics 19 276-288

[170] Heusler M 1995 Class.Quant.Grav. 12 2021-2036 [arXiv:gr-qc/9503053]

[171] Sotiriou T P and Faraoni V 2012 Phys.Rev.Lett. 108081103 [arXiv:1109.6324]

[172] Graham A A H and Jha R 2014 Phys.Rev. D90 041501 [arXiv:1407.6573]

[173] Hui L and Nicolis A 2013 Phys.Rev.Lett. 110241104 [arXiv:1202.1296]

[174] Campbell B A, Kaloper N and Olive K A 1992 Physics Letters B 285 199-205

[175] Mignemi S and Stewart N 1993 Phys.Rev. D47 5259-5269 [arXiv:hep-th/9212146]

[176] Yunes N and Stein L C 2011 Phys.Rev. D83 104002 [arXiv:1101.2921]

[177] Sotiriou T P and Zhou S Y 2014 Phys.Rev.Lett. 112251102 [arXiv:1312.3622]

[178] Sotiriou T P and Zhou S Y 2014 Phys.Rev. D90 124063 [arXiv:1408.1698]

[179] Cardoso V, Chakrabarti S, Pani P, Berti E and Gualtieri L 2011 Phys.Rev.Lett. 107241101 [arXiv:1109.6021]

[180] Barausse E, Palenzuela C, Ponce M and Lehner L 2013 Phys.Rev. D87 081506 [arXiv:1212.5053]

[181] Palenzuela C, Barausse E, Ponce M and Lehner L 2014 Phys.Rev. D89 044024 [arXiv:1310.4481]

[182] Shibata M, Taniguchi K, Okawa H and Buonanno A 2014 Phys.Rev. D89 084005 [arXiv:1310.0627]

[183] Taniguchi K, Shibata M and Buonanno A 2015 Phys.Rev. D91 024033 [arXiv:1410.0738]

[184] Sampson L, Yunes N, Cornish N, Ponce M, Barausse E et al. 2014 Phys.Rev. D90 124091 [arXiv:1407.7038]

[185] Bhat N D R, Bailes M and Verbiest J P W 2008 Phys. Rev. D77 124017 [arXiv:0804.0956]

[186] Freire P C et al. 2012 Mon.Not.Roy.Astron.Soc. 4233328 [arXiv:1205.1450]

[187] Antoniadis J, Freire P C, Wex N, Tauris T M, Lynch R S et al. 2013 Science 3406131 [arXiv:1304.6875]

[188] Alsing J, Berti E, Will C M and Zaglauer H 2012 Phys.Rev. D85 064041 [arXiv:1112.4903]

[189] Berti E et al. 2015 Class. Quant. Grav. 32243001 [arXiv:1501.07274]

[190] Bertotti B, Iess L and Tortora P 2003 Nature 425374 
[191] Perivolaropoulos L 2010 Phys. Rev. D81 047501 [arXiv:0911.3401]

[192] Liu K, Eatough R, Wex N and Kramer M 2014 Mon.Not.Roy.Astron.Soc. 4453115 [arXiv:1409.3882]

[193] Fierz M and Pauli W 1939 Proc. Roy. Soc. Lond. A173 211-232

[194] van Dam H and Veltman M J G 1970 Nucl. Phys. B22 397-411

[195] Zakharov V I 1970 JETP Lett. 12312 [Pisma Zh.Eksp.Teor.Fiz.12:447-449,1970]

[196] Boulware D G and Deser S 1972 Phys. Rev. D6 3368-3382

[197] de Rham C and Gabadadze G 2010 Phys. Rev. D82 044020 [arXiv:1007.0443]

[198] de Rham C, Gabadadze G and Tolley A J 2011 Phys. Rev. Lett. 106231101 [arXiv:1011.1232]

[199] Hassan S F and Rosen R A 2011 JHEP 07009 [arXiv:1103.6055]

[200] Talmadge C, Berthias J P, Hellings R W and Standish E M 1988 Phys. Rev. Lett. 61 1159-1162

[201] Finn L S and Sutton P J 2002 Phys. Rev. D65 044022 [arXiv:gr-qc/0109049]

[202] Goldhaber A S and Nieto M M 1974 Phys. Rev. D9 1119-1121

[203] Choudhury S R, Joshi G C, Mahajan S and McKellar B H J 2004 Astropart. Phys. 21 559-563 [arXiv:hep-ph/0204161]

[204] Sjors S and Mortsell E 2013 JHEP 1302080 [arXiv:1111.5961]

[205] Kocsis B, Haiman Z and Menou K 2008 Astrophys. J. 684 870-887 [arXiv:0712.1144]

[206] Hazboun J S and Larson S L 2013 [arXiv:1311.3153]

[207] Nishizawa A and Nakamura T 2014 Phys.Rev. D90 044048 [arXiv:1406.5544]

[208] Metsaev R and Tseytlin A A 1987 Nucl.Phys. B293 385-419

[209] Maeda K i, Ohta N and Sasagawa Y 2009 Phys.Rev. D80 104032 [arXiv:0908.4151]

[210] Jackiw R and Pi S 2003 Phys.Rev. D68 104012 [arXiv:gr-qc/0308071]

[211] Smith T L, Erickcek A L, Caldwell R R and Kamionkowski M 2008 Phys. Rev. D77 024015 [arXiv:0708.0001]

[212] Alexander S and Yunes N 2009 Phys.Rept. 480 1-55 [arXiv:0907.2562]

[213] Yagi K 2012 Phys.Rev. D86 081504 [arXiv:1204.4524]

[214] Yagi K, Stein L C and Yunes N 2016 Phys. Rev. D93 024010 [arXiv:1510.02152]

[215] Randall L and Sundrum R 1999 Phys. Rev. Lett. 83 4690-4693 [arXiv:hep-th/9906064]

[216] Garriga J and Tanaka T 2000 Phys. Rev. Lett. 84 2778-2781 [arXiv:hep-th/9911055]

[217] Adelberger E G et al. 2007 Phys. Rev. Lett. 98131104 [arXiv:hep-ph/0611223]

[218] Emparan R, Fabbri A and Kaloper N 2002 JHEP 08043 [arXiv:hep-th/0206155]

[219] Tanaka T 2003 Prog. Theor. Phys. Suppl. 148 307-316 [arXiv:gr-qc/0203082]

[220] Maldacena J M 1998 Adv. Theor. Math. Phys. 2 231-252 [arXiv:hep-th/9711200]

[221] Aharony O, Gubser S S, Maldacena J M, Ooguri H and Oz Y 2000 Phys. Rept. 323 183-386 [arXiv:hep-th/9905111]

[222] Gregory R 2000 Class. Quant. Grav. 17 L125-L132 [arXiv:hep-th/0004101]

[223] Johannsen T, Psaltis D and McClintock J E 2009 Astrophys. J. 691 997-1004 [arXiv:0803.1835]

[224] Johannsen T 2009 Astron. and Astrophys. 507 617-620 [arXiv:0812.0809]

[225] Figueras P and Wiseman T 2011 Phys.Rev.Lett. 107081101 [arXiv:1105.2558]

[226] Abdolrahimi S, Cattoen C, Page D N and Yaghoobpour-Tari S 2013 Phys.Lett. B720 405-409 [arXiv:1206.0708]

[227] Yunes N, Pretorius F and Spergel D 2010 Phys. Rev. D81 064018 [arXiv:0912.2724]

[228] Babichev E, Dokuchaev V and Eroshenko Y 2004 Phys.Rev.Lett. 93021102 [arXiv:gr-qc/0402089]

[229] Babichev E, Dokuchaev V and Eroshenko Y 2005 J.Exp.Theor.Phys. 100 528-538 [arXiv:astro$\mathrm{ph} / 0505618]$

[230] Nordtvedt K 1968 Phys.Rev. 169 1017-1025

[231] Damour T and Deruelle N 1986 Ann. Inst. Henri Poincaré Phys. Théor., Vol. 44, No. 3, p. 263 29244 263-292 URL http://www.numdam.org/item?id=AIHPA_1986__44_3_263_0

[232] Damour T and Taylor J H 1992 Phys.Rev. D45 1840-1868

[233] Taylor J H and Weisberg J 1989 Astrophys.J. 345 434-450

[234] Hu W and Sawicki I 2007 Phys.Rev. D76 104043 [arXiv:0708.1190] 
[235] Baker T, Ferreira P G and Skordis C 2013 Phys.Rev. D87 024015 [arXiv:1209.2117]

[236] Li Y H, Zhang J F and Zhang X 2014 Phys.Rev. D90 063005 [arXiv:1404.5220]

[237] Montgomery C, Orchiston W and Whittingham I 2009 Journal of Astronomical History and Heritage 12 90-96

[238] Price R H 1972 Phys. Rev. D5 2419-2438

[239] Geroch R P 1970 J.Math.Phys. 11 1955-1961

[240] Geroch R P 1970 J.Math.Phys. 11 2580-2588

[241] Wald R 1984 General Relativity (Chicago: The University of Chicago Press)

[242] Vigeland S, Yunes N and Stein L 2011 Phys. Rev. D83 104027 [arXiv:1102.3706]

[243] Manko V S and Novikov I D 1992 Classical and Quantum Gravity 9 2477-2487

[244] Backdahl T and Herberthson M 2005 Class.Quant.Grav. 22 1607-1621 [arXiv:gr-qc/0502012]

[245] Backdahl T and Herberthson M 2005 Class.Quant.Grav. 22 3585-3594 [arXiv:gr-qc/0506086]

[246] Backdahl T 2007 Class.Quant.Grav. 24 2205-2215 [arXiv:gr-qc/0612043]

[247] Johannsen T 2013 Phys.Rev. D87 124017 [arXiv:1304.7786]

[248] Collins N A and Hughes S A 2004 Phys. Rev. D69 124022 [arXiv:gr-qc/0402063]

[249] Vigeland S J and Hughes S A 2010 Phys. Rev. D81 024030 [arXiv:0911.1756]

[250] Vigeland S J 2010 Phys.Rev. D82 104041 [arXiv:1008.1278]

[251] Suen W, Price R and Redmount I 1988 Phys.Rev. D37 2761-2789

[252] Newman E and Janis A 1965 J.Math.Phys. 6 915-917

[253] Hansen D and Yunes N 2013 Phys.Rev. D88 104020 [arXiv:1308.6631]

[254] Hartle J B and Thorne K S 1968 Astrophys.J. 153807

[255] Johannsen T and Psaltis D 2010 Astrophys.J. 716 187-197 [arXiv:1003.3415]

[256] Johannsen T and Psaltis D 2011 Phys.Rev. D83 124015 [arXiv:1105.3191]

[257] Cardoso V, Pani P and Rico J 2014 Phys.Rev. D89 064007 [arXiv:1401.0528]

[258] Rezzolla L and Zhidenko A 2014 Phys.Rev. D90 084009 [arXiv:1407.3086]

[259] Yunes N and Pretorius F 2009 Phys.Rev. D79 084043 [arXiv:0902.4669]

[260] Yagi K, Yunes N and Tanaka T 2012 Phys.Rev. D86 044037 [arXiv:1206.6130]

[261] Johannsen T 2013 Phys.Rev. D88 044002 [arXiv:1501.02809]

[262] Maggiore M 2007 Gravitational Waves: Volume 1: Theory and Experiments (OUP Oxford) ISBN 9780198570745 URL https://books.google.it/books?id=AqVpQgAACAAJ

[263] Arun K G, Iyer B R, Sathyaprakash B S and Sundararajan P A 2005 Phys. Rev. D71 084008 [Erratum-ibid.D72:069903,2005] [arXiv:gr-qc/0411146]

[264] Finn L S 1992 Phys. Rev. D46 5236-5249 [arXiv:gr-qc/9209010]

[265] Cutler C and Flanagan E E 1994 Phys. Rev. D49 2658-2697 [arXiv:gr-qc/9402014]

[266] Cornish N J and Littenberg T B 2007 Phys.Rev. D76 083006 [arXiv:0704.1808]

[267] Littenberg T B and Cornish N J 2009 Phys.Rev. D80 063007 [arXiv:0902.0368]

[268] Veitch J, Raymond V, Farr B, Farr W, Graff P et al. 2015 Phys.Rev. D91 042003 [arXiv:1409.7215]

[269] Yunes N and Hughes S A 2010 Phys.Rev. D82 082002 [arXiv:1007.1995]

[270] Burgay M, D’Amico N, Possenti A, Manchester R N, Lyne A G, Joshi B C, McLaughlin M A, Kramer M, Sarkissian J M, Camilo F, Kalogera V, Kim C and Lorimer D R 2003 Nature 426 531-533

[271] Lyne A G, Burgay M, Kramer M, Possenti A, Manchester R N, Camilo F, McLaughlin M A, Lorimer D R, D'Amico N, Joshi B C, Reynolds J and Freire P C C 2004 Science 303 1153-1157 [arXiv:arXiv:astro-ph/0401086]

[272] Kramer M, Stairs I H, Manchester R, McLaughlin M, Lyne A et al. 2006 Science 314 97-102 [arXiv:astro-ph/0609417]

[273] Kramer M and Wex N 2009 Class.Quant.Grav. 26073001

[274] Barack L and Cutler C 2004 Phys. Rev. 69082005 [arXiv:gr-qc/0310125]

[275] Hartle J B 1967 Astrophys.J. 150 1005-1029

[276] Liebling S L and Palenzuela C 2012 Living Rev.Rel. 156 [arXiv:1202.5809]

[277] Palenzuela C, Olabarrieta I, Lehner L and Liebling S L 2007 Phys.Rev. D75 064005 [arXiv:gr- 
qc/0612067]

[278] Palenzuela C, Lehner L and Liebling S L 2008 Phys.Rev. D77 044036 [arXiv:0706.2435]

[279] Mazur P O and Mottola E 2001 [arXiv:gr-qc/0109035]

[280] Berti E, Cardoso V and Starinets A O 2009 Class.Quant.Grav. 26163001 [arXiv:0905.2975]

[281] Ward W R 1997 Icarus 126 261-281

[282] Armitage P J 2007 [arXiv:astro-ph/0701485]

[283] Heckman T M, Kauffmann G, Brinchmann J, Charlot S, Tremonti C and White S D M 2004 Astrophys. J. 613 109-118 [arXiv:astro-ph/0406218]

[284] Kelly B C, Vestergaard M, Fan X, Hopkins P, Hernquist L and Siemiginowska A 2010 Astrophys. J. 719 1315-1334 [arXiv:1006.3561]

[285] Papaloizou J and Pringle J E 1977 Mon. Not. Roy. Astron. Soc. 181 441-454

[286] Goldreich P and Tremaine S 1979 Astrophys. J. 233 857-871

[287] Artymowicz P and Lubow S H 1994 Astrophys. J. 421 651-667

[288] Hayasaki K, Mineshige S and Sudou H 2007 PASJ 59 427-441 [arXiv:arXiv:astro-ph/0609144]

[289] Shi J M, Krolik J H, Lubow S H and Hawley J F 2012 Astrophys. J. 749118 [arXiv:1110.4866]

[290] Bondi H and Hoyle F 1944 Mon. Not. Roy. Astron. Soc. 104273

[291] Seto N, Kawamura S and Nakamura T 2001 Phys. Rev. Lett. 87221103 [arXiv:astro-ph/0108011]

[292] Takahashi R and Nakamura T 2005 Prog. Theor. Phys. 113 63-71 [arXiv:astro-ph/0408547]

[293] Nishizawa A, Yagi K, Taruya A and Tanaka T 2012 Phys. Rev. D85 044047 [arXiv:1110.2865]

[294] Yagi K, Nishizawa A and Yoo C M 2012 JCAP 1204031 [arXiv:1112.6040]

[295] Konno K, Matsuyama T and Tanda S 2009 Prog.Theor.Phys. 122 561-568 [arXiv:0902.4767]

[296] Yagi K, Stein L C, Yunes N and Tanaka T 2013 Phys.Rev. D87 084058 [arXiv:1302.1918]

[297] Will C M and Wiseman A G 1996 Phys.Rev. D54 4813-4848 [arXiv:gr-qc/9608012]

[298] Cornish N, Sampson L, Yunes N and Pretorius F 2011 Phys.Rev. D84 062003 [arXiv:1105.2088]

[299] Psaltis D and Johannsen T 2012 Astrophys.J. 7451 [arXiv:1011.4078]

[300] Vincent F, Paumard T, Gourgoulhon E and Perrin G 2011 Class.Quant.Grav. 28225011 [arXiv:1109.4769]

[301] Krawczynski H 2012 Astrophys. J. 754133 [arXiv:1205.7063]

[302] Liu D, Li Z, Cheng Y and Bambi C 2015 Eur. Phys. J. C75 383 [arXiv:1504.06788]

[303] Wex N and Kopeikin S M 1999 Astrophys. J. 514 388-401 [arXiv:astro-ph/9811052]

[304] Will C M 2008 Astrophys. J. Lett. 674 L25-L28 [arXiv:0711.1677]

[305] Merritt D, Alexander T, Mikkola S and Will C M 2010 Phys. Rev. D81 062002 [arXiv:0911.4718]

[306] Sadeghian L and Will C M 2011 Class. Quant. Grav. 28225029 [arXiv:1106.5056]

[307] Liu K, Wex N, Kramer M, Cordes J M and Lazio T J W 2012 Astrophys. J. 7471 [arXiv:1112.2151]

[308] Li Z, Kong L and Bambi C 2014 Astrophys. J. 787152 [arXiv:1401.1282]

[309] Li Z and Bambi C 2014 Phys. Rev. D90 024071 [arXiv:1405.1883]

[310] Liu D, Li Z and Bambi C 2015 JCAP 1501020 [arXiv:1411.2329]

[311] Bambi C 2012 Phys. Rev. D85 043002 [arXiv:1201.1638]

[312] Bambi C 2012 Phys. Rev. D86 123013 [arXiv:1204.6395]

[313] Novikov I D and Thorne K S 1973 Astrophysics of black holes. Black Holes (Les Astres Occlus) ed Dewitt C and Dewitt B S pp 343-450

[314] Kovacs Z and Harko T 2010 Phys.Rev. D82 124047 [arXiv:1011.4127]

[315] Takahashi R and Harada T 2010 Class.Quant.Grav. 27075003 [arXiv:1002.0421]

[316] Steiner J F, McClintock J E, Remillard R A, Gou L, Yamada S and Narayan R 2010 Astrophys. J. 718 L117-L121 [arXiv:1006.5729]

[317] Fabian A C, Rees M J, Stella L and White N E 1989 Mon. Not. Roy. Astron. Soc. $238729-736$

[318] Tanaka Y, Nandra K, Fabian A C, Inoue H, Otani C, Dotani T, Hayashida K, Iwasawa K, Kii T, Kunieda H, Makino F and Matsuoka M 1995 Nature 375 659-661

[319] Reynolds C S and Nowak M A 2003 Phys.Rept. 377 389-466 [arXiv:astro-ph/0212065]

[320] Miller J M 2007 ARA \& A 45 441-479 [arXiv:0705.0540]

[321] Moore C J and Gair J R 2015 Phys. Rev. D92 024039 [arXiv:1507.02998] 
[322] Bambi C 2012 Astrophys.J. 761174 [arXiv:1210.5679]

[323] Falcke H, Melia F and Agol E 2000 Astrophys.J. 528 L13 [arXiv:astro-ph/9912263]

[324] Takahashi R 2004 J.Korean Phys.Soc. 45 S1808-S1812 [arXiv:astro-ph/0405099]

[325] Takahashi R 2005 Publ.Astron.Soc.Jap. 57273 [arXiv:astro-ph/0505316]

[326] Huang L, Cai M, Shen Z Q and Yuan F 2007 Mon.Not.Roy.Astron.Soc. 379 833-840 [arXiv:astro$\mathrm{ph} / 0703254$

[327] Broderick A and Loeb A 2009 Astrophys.J. 697 1164-1179 [arXiv:0812.0366]

[328] Bambi C and Freese K 2009 Phys.Rev. D79 043002 [arXiv:0812.1328]

[329] Hioki K and Maeda K i 2009 Phys.Rev. D80 024042 [arXiv:0904.3575]

[330] Bambi C and Yoshida N 2010 Class.Quant.Grav. 27205006 [arXiv:1004.3149]

[331] Takahashi R and Mineshige S 2011 Astrophys.J. 72986 [arXiv:1102.3583]

[332] Lu R S, Broderick A E, Baron F, Monnier J D, Fish V L et al. 2014 Astrophys.J. 788120 [arXiv:1404.7095]

[333] Cunha P V P, Herdeiro C A R, Radu E and Runarsson H F 2015 Phys. Rev. Lett. 115211102 [arXiv:1509.00021]

[334] Ghasemi-Nodehi M, Li Z and Bambi C 2015 Eur. Phys. J. C75 315 [arXiv:1506.02627]

[335] Mashhoon B 1985 Phys. Rev. D31 290-293

[336] Cardoso V, Miranda A S, Berti E, Witek H and Zanchin V T 2009 Phys. Rev. D79 064016 [arXiv:0812.1806]

[337] McClintock J E and Remillard R A 2006 Black hole binaries Compact stellar X-ray sources ed Lewin W H G and van der Klis M pp 157-213 ISBN 9780521826594

[338] Lewin W H G and van der Klis M 2006 Compact stellar X-ray sources (Cambridge University Press) ISBN 9780521826594

[339] Abramowicz M A and Kluzniak W 2001 A 83 A 374 L19-L20 [arXiv:astro-ph/0105077]

[340] Aliev A N and Galtsov D V 1981 Gen. Rel. Grav. 13 899-912

[341] Aliev A N, Galtsov D V and Petukhov V I 1986 Astr. Space Sci. 124 137-157

[342] Torok G, Kotrlova A, Sramkova E and Stuchlik Z 2011 Astron.Astrophys. 531 A59 [arXiv:1103.2438]

[343] Aliev A N, Esmer G D and Talazan P 2013 Class.Quant.Grav. 30045010 [arXiv:1205.2838]

[344] Stella L and Vietri M 1998 Astrophys.J. 492 L59 [arXiv:astro-ph/9709085]

[345] Stella L and Vietri M 1999 Phys.Rev.Lett. 82 17-20 [arXiv:astro-ph/9812124]

[346] Morsink S M and Stella L 1999 Astrophys.J. 513 827-844 [arXiv:astro-ph/9808227]

[347] Stella L, Vietri M and Morsink S 1999 Astrophys.J. 524 L63-L66 [arXiv:astro-ph/9907346]

[348] Strohmayer T E 2001 Astrophys.J. 552 L49-L53 [arXiv:astro-ph/0104487]

[349] Casella P, Belloni T and Stella L 2005 Astrophys.J. 629 403-407 [arXiv:astro-ph/0504318]

[350] Motta S, Homan J, Munoz-Darias T, Casella P, Belloni T et al. 2012 Mon.Not.Roy.Astron.Soc. 427595 [arXiv:1209.0327]

[351] Motta S, Belloni T, Stella L, Munoz-Darias T and Fender R 2014 Mon.Not.Roy.Astron.Soc. 437 2554 [arXiv:1309.3652]

[352] Beer M E and Podsiadlowski P 2002 Mon.Not.Roy.Astron.Soc. 331351 [arXiv:astro-ph/0109136]

[353] Shafee R, McClintock J E, Narayan R, Davis S W, Li L X et al. 2006 Astrophys.J. 636 L113-L116 [arXiv:astro-ph/0508302]

[354] Čadež A, Calvani M and Kostić U 2008 A \& $A 487$ 527-532 [arXiv:0809.1783]

[355] Kostić U, Čadež A, Calvani M and Gomboc A 2009 A \&3 A 496 307-315 [arXiv:0901.3447]

[356] Abramowicz M A, Karas V, Kluzniak W, Lee W H and Rebusco P 2003 Publ. Astron. Soc. Jap. 55 467-466 [arXiv:astro-ph/0302183]

[357] Török G, Abramowicz M A, Kluźniak W and Stuchlík Z 2005 A \& A 436 1-8

[358] Kato S 2001 Publ. Astron. Soc. Jap. 53 1-24

[359] Kato S 2004 Publ. Astron. Soc. Jap. 56 559-567

[360] Kato S 2004 Publ. Astron. Soc. Jap. 56 905-922 [arXiv:astro-ph/0409051]

[361] Kato S 2005 Publ. Astron. Soc. Jap. 57 699-703 [arXiv:astro-ph/0507234] 
[362] Kato S 2008 Publ. Astron. Soc. Jap. 60 111- [arXiv:0709.2467]

[363] Bursa M, Abramowicz M A, Karas V and Kluźniak W 2004 Astrophys. J. Lett. 617 L45-L48 [arXiv:astro-ph/0406586]

[364] Török G, Bakala P, Šrámková E, Stuchlík Z and Urbanec M 2010 Astrophys. J. $714748-757$ [arXiv:1008.0088]

[365] Kato S and Fukue J 1980 Publ. Astron. Soc. Jap. 32377

[366] Okazaki A T, Kato S and Fukue J 1987 Publ. Astron. Soc. Jap. 39 457-473

[367] Nowak M A and Wagoner R V 1992 Astrophys. J. 393 697-707

[368] Perez C A, Silbergleit A S, Wagoner R V and Lehr D E 1997 Astrophys.J. 476 589-604 [arXiv:astro-ph/9601146]

[369] Silbergleit A S, Wagoner R V and Ortega-Rodríguez M 2001 Astrophys. J. 548 335-347 [arXiv:astro-ph/0004114]

[370] Wagoner R V, Silbergleit A S and Ortega-Rodríguez M 2001 Astrophys. J. Lett. 559 L25-L28 [arXiv:astro-ph/0107168]

[371] Ortega-Rodríguez M, Silbergleit A S and Wagoner R V 2002 Astrophys. J. 567 1043-1056 [arXiv:astro-ph/0611010]

[372] Silbergleit A S and Wagoner R V 2008 Astrophys. J. 680 1319-1325 [arXiv:0711.4848]

[373] Tsang D and Lai D 2009 Mon. Not. Roy. Astron. Soc. 393 992-998 [arXiv:0810.1299]

[374] Fu W and Lai D 2009 Astrophys. J. 690 1386-1392 [arXiv:0806.1938]

[375] Fu W and Lai D 2011 Mon. Not. Roy. Astron. Soc. 410 399-416 [arXiv:1006.3763]

[376] Wambsganss J, Paczynski B and Schneider P 1990 Astrophys. J. Lett. 358 L33-L36

[377] Rauch K P and Blandford R D 1991 Astrophys. J. Lett. 381 L39-L42

[378] Pooley D, Blackburne J A, Rappaport S and Schechter P L 2007 Astrophys. J. 661 19-29 [arXiv:astro-ph/0607655]

[379] Dai X, Kochanek C S, Chartas G, Kozlowski S, Morgan C W, Garmire G and Agol E 2010 Astrophys. J. 709 278-285 [arXiv:0906.4342]

[380] Morgan C W, Kochanek C S, Morgan N D and Falco E E 2010 Astrophys. J. 712 1129-1136 [arXiv:1002.4160]

[381] Blackburne J A and Kochanek C S 2010 Astrophys. J. 718 1079-1084 [arXiv:1002.3126]

[382] Jimenez-Vicente J, Mediavilla E, Munoz J A and Kochanek C S 2012 Astrophys. J. 751106 [arXiv:1201.3187]

[383] Jimenez-Vicente J, Mediavilla E, Kochanek C S, Munoz J A, Motta V, Falco E and Mosquera A M 2014 Astrophys. J. 78347 [arXiv:1401.2785]

[384] Miller M C and Miller J M 2014 Phys. Rept. 548 1-34 [arXiv:1408.4145]

[385] Davis S W, Done C and Blaes O M 2006 Astrophys. J. 647 525-538 [arXiv:astro-ph/0602245]

[386] Fragos T, Tremmel M, Rantsiou E and Belczynski K 2010 Astrophys. J. Lett. 719 L79-L83 [arXiv:1001.1107]

[387] Bardeen J M and Petterson J A 1975 Astrophys. J. 195 L65

[388] Martin R G, Tout C A and Pringle J E 2008 Mon. Not. Roy. Astron. Soc. 387188 [arXiv:0802.3912]

[389] Maccarone T J 2002 Mon. Not. Roy. Astron. Soc. 3361371 [arXiv:astro-ph/0209105]

[390] Fragile P C, Mathews G J and Wilson J R 2001 Astrophys. J. 553955 [arXiv:astro-ph/0007478]

[391] Steiner J F and McClintock J E 2012 Astrophys. J. 745136 [arXiv:1110.6849]

[392] Hjellming R M and Rupen M P 1995 Nature 375 464-468

[393] Greene J, Bailyn C D and Orosz J A 2001 Astrophys. J. 5541290 [arXiv:astro-ph/0101337]

[394] Orosz J A, Kuulkers E, van der Klis M, McClintock J E, Garcia M R, Callanan P J, Bailyn C D, Jain R K and Remillard R A 2001 Astrophys. J. 555489 [arXiv:astro-ph/0103045]

[395] Li L X, Zimmerman E R, Narayan R and McClintock J E 2005 Astrophys. J. Suppl. 157 335-370 [arXiv:astro-ph/0411583]

[396] Davis S W, Blaes O M, Hubeny I and Turner N J 2005 Astrophys. J. 621 372-387 [arXiv:astro$\mathrm{ph} / 0408590]$

[397] Noble S C, Krolik J H, Schnittman J D and Hawley J F 2011 Astrophys. J. 743115 
[arXiv:1105.2825]

[398] Beckwith K, Hawley J and Krolik J 2008 Mon. Not. Roy. Astron. Soc. 39021 [arXiv:0801.2974]

[399] Noble S C, Krolik J H and Hawley J F 2009 Astrophys. J. 692 411-421 [arXiv:0808.3140]

[400] Horndeski G W 1974 Int.J.Theor.Phys. 10 363-384

[401] Deffayet C, Gao X, Steer D and Zahariade G 2011 Phys.Rev. D84 064039 [arXiv:1103.3260]

[402] Bergmann P G 1968 Int.J.Theor.Phys. 1 25-36

[403] Wagoner R V 1970 Phys.Rev. D1 3209-3216

[404] Brans C and Dicke R H 1961 Phys. Rev. 124 925-935

[405] Damour T and Esposito-Farese G 1992 Class.Quant.Grav. 9 2093-2176

[406] Damour T and Esposito-Farese G 1993 Phys.Rev.Lett. 70 2220-2223

[407] Damour T and Esposito-Farese G 1996 Phys.Rev. D54 1474-1491 [arXiv:gr-qc/9602056]

[408] Jacobson T 1999 Phys.Rev.Lett. 83 2699-2702 [arXiv:astro-ph/9905303]

[409] Pena I and Sudarsky D 1997 Class.Quant.Grav. 14 3131-3134

[410] Herdeiro C A R and Radu E 2014 Phys.Rev.Lett. 112221101 [arXiv:1403.2757]

[411] Herdeiro C and Radu E 2015 Class. Quant. Grav. 32144001 [arXiv:1501.04319]

[412] Herdeiro C A R, Radu E and Rúnarsson H 2015 Phys. Rev. D92 084059 [arXiv:1509.02923]

[413] Herdeiro C A R and Radu E 2015 Int. J. Mod. Phys. D24 1542014 [arXiv:1504.08209]

[414] Cardoso V, Carucci I P, Pani P and Sotiriou T P 2013 Phys.Rev.Lett. 111111101 [arXiv:1308.6587]

[415] Cardoso V, Carucci I P, Pani P and Sotiriou T P 2013 Phys.Rev. D88 044056 [arXiv:1305.6936]

[416] Babichev E and Charmousis C 2014 JHEP 1408106 [arXiv:1312.3204]

[417] Kobayashi T, Motohashi H and Suyama T 2012 Phys.Rev. D85 084025 [arXiv:1202.4893]

[418] Kobayashi T, Motohashi H and Suyama T 2014 Phys.Rev. D89 084042 [arXiv:1402.6740]

[419] Will C M 1977 Astrophys. J. 214 826-839

[420] Will C M and Zaglauer H W 1989 Astrophys. J. 346366

[421] Mirshekari S and Will C M 2013 Phys.Rev. D87 084070 [arXiv:1301.4680]

[422] Lang R N 2014 Phys.Rev. D89 084014 [arXiv:1310.3320]

[423] Lang R N 2015 Phys.Rev. D91 084027 [arXiv:1411.3073]

[424] Eardley D M 1975 Astrophys. J. 196 L59-L62

[425] Sahni V and Wang L M 2000 Phys.Rev. D62 103517 [arXiv:astro-ph/9910097]

[426] Hu W, Barkana R and Gruzinov A 2000 Phys.Rev.Lett. 85 1158-1161 [arXiv:astro-ph/0003365]

[427] Nan R, Li D, Jin C, Wang Q, Zhu L et al. 2011 Int.J.Mod.Phys. D20 989-1024 [arXiv:1105.3794]

[428] Carilli C L and Rawlings S 2004 New Astron.Rev. 48979 [arXiv:astro-ph/0409274]

[429] Horbatsch M and Burgess C 2012 JCAP 1205010 [arXiv:1111.4009]

[430] Valtonen M J, Lehto H J, Nilsson K, Heidt J, Takalo L O, Sillanpää A, Villforth C, Kidger M, Poyner G, Pursimo T, Zola S, Wu J H, Zhou X, Sadakane K, Drozdz M, Koziel D, Marchev D, Ogloza W, Porowski C, Siwak M, Stachowski G, Winiarski M, Hentunen V P, Nissinen M, Liakos A and Dogru S 2008 Nature 452 851-853 [arXiv:0809.1280]

[431] Rubakov V A and Tinyakov P G 2008 Phys. Usp. 51 759-792 [arXiv:0802.4379]

[432] Hinterbichler K 2012 Rev. Mod. Phys. 84 671-710 [arXiv:1105.3735]

[433] de Rham C 2014 Living Rev.Rel. 177 [arXiv:1401.4173]

[434] Vainshtein A I 1972 Phys. Lett. B39 393-394

[435] Dvali G R, Gabadadze G and Porrati M 2000 Phys. Lett. B485 208-214 [arXiv:hep-th/0005016]

[436] Nicolis A and Rattazzi R 2004 JHEP 06059 [arXiv:hep-th/0404159]

[437] Creminelli P, Nicolis A, Papucci M and Trincherini E 2005 JHEP 0509003 [arXiv:hep-th/0505147]

[438] Rubakov V A 2004 [arXiv:hep-th/0407104]

[439] Dubovsky S L 2004 JHEP 10076 [arXiv:hep-th/0409124]

[440] Blas D and Sibiryakov S 2015 Zh.Eksp.Teor.Fiz. 147 578-594 [arXiv:1410.2408]

[441] Chamseddine A H and Mukhanov V 2010 JHEP 08011 [arXiv:1002.3877]

[442] Alberte L, Chamseddine A H and Mukhanov V 2010 JHEP 12023 [arXiv:1008.5132]

[443] Chamseddine A H and Mukhanov V 2011 JHEP 08091 [arXiv:1106.5868]

[444] Hassan S and Rosen R A 2012 Phys.Rev.Lett. 108041101 [arXiv:1106.3344] 
[445] Hassan S, Rosen R A and Schmidt-May A 2012 JHEP 1202026 [arXiv:1109.3230]

[446] Hassan S and Rosen R A 2012 JHEP 1202126 [arXiv:1109.3515]

[447] Hassan S and Rosen R A 2012 JHEP 1204123 [arXiv:1111.2070]

[448] de Rham C, Gabadadze G and Tolley A J 2012 Phys.Lett. B711 190-195 [arXiv:1107.3820]

[449] de Rham C, Gabadadze G and Tolley A J 2011 JHEP 11093 [arXiv:1108.4521]

[450] Huang Q G, Piao Y S and Zhou S Y 2012 Phys.Rev. D86 124014 [arXiv:1206.5678]

[451] Huang Q G, Zhang K C and Zhou S Y 2013 JCAP 1308050 [arXiv:1306.4740]

[452] De Felice A and Mukohyama S 2014 Phys.Lett. B728 622-625 [arXiv:1306.5502]

[453] De Felice A, Emir Gumrukcuoglu A and Mukohyama S 2013 Phys.Rev. D88 124006 [arXiv:1309.3162]

[454] Mukohyama S 2013 [arXiv:1309.2146]

[455] Deffayet C and Jacobson T 2012 Class. Quant. Grav. 29065009 [arXiv:1107.4978]

[456] Babichev E and Fabbri A 2014 Phys.Rev. D90 084019 [arXiv:1406.6096]

[457] Babichev E and Fabbri A 2013 Class.Quant.Grav. 30152001 [arXiv:1304.5992]

[458] Brito R, Cardoso V and Pani P 2013 Phys.Rev. D88 023514 [arXiv:1304.6725]

[459] Brito R, Cardoso V and Pani P 2013 Phys.Rev. D87 124024 [arXiv:1306.0908]

[460] Brito R, Cardoso V and Pani P 2013 Phys.Rev. D88 064006 [arXiv:1309.0818]

[461] Volkov M S 2013 Class. Quant. Grav. 30184009 [arXiv:1304.0238]

[462] Tasinato G, Koyama K and Niz G 2013 Class. Quant. Grav. 30184002 [arXiv:1304.0601]

[463] Volkov M S 2015 Lect.Notes Phys. 892 161-180 [arXiv:1405.1742]

[464] Babichev E and Brito R 2015 Class. Quant. Grav. 32154001 [arXiv:1503.07529]

[465] Jiménez J B, Piazza F and Velten H 2016 Phys. Rev. Lett. 116061101 [arXiv:1507.05047]

[466] Apostolatos T A, Cutler C, Sussman G J and Thorne K S 1994 Phys. Rev. D49 6274-6297

[467] Ajith P et al. 2008 Phys. Rev. D77 104017 [Erratum-ibid.D79:129901,2009] [arXiv:0710.2335]

[468] Ajith P et al. 2011 Phys. Rev. Lett. 106241101 [arXiv:0909.2867]

[469] Amelino-Camelia G 2001 Phys.Lett. B510 255-263 [arXiv:hep-th/0012238]

[470] Magueijo J and Smolin L 2002 Phys.Rev.Lett. 88190403 [arXiv:hep-th/0112090]

[471] Amelino-Camelia G 2002 Nature 418 34-35 [arXiv:gr-qc/0207049]

[472] Amelino-Camelia G 2010 Symmetry 2 230-271 [arXiv:1003.3942]

[473] Sefiedgar A, Nozari K and Sepangi H 2011 Phys.Lett. B696 119-123 [arXiv:1012.1406]

[474] Hořava P 2009 JHEP 0903020 [arXiv:0812.4287]

[475] Hořava P 2009 Phys.Rev. D79 084008 [arXiv:0901.3775]

[476] Garattini R and Mandanici G 2011 Phys.Rev. D83 084021 [arXiv:1102.3803]

[477] Collins J, Perez A, Sudarsky D, Urrutia L and Vucetich H 2004 Phys. Rev. Lett. 93191301 [arXiv:gr-qc/0403053]

[478] Collins J, Perez A and Sudarsky D 2009 Lorentz invariance violation and its role in quantum gravity phenomenology Approaches to Quantum Gravity ed Oriti D (Cambridge University Press) pp 528-547 ISBN 9780521860451 [arXiv:hep-th/0603002]

[479] Hansen D, Yunes N and Yagi K 2015 Phys.Rev. D91 082003 [arXiv:1412.4132]

[480] Rovelli C 2008 Living Rev.Rel. 115

[481] Rovelli C 2011 Class.Quant.Grav. 28153002 [arXiv:1012.4707]

[482] Gross D J and Sloan J H 1987 Nucl.Phys. B291 41-89

[483] Mignemi S 1995 Phys.Rev. D51 934-937 [arXiv:hep-th/9303102]

[484] Kleihaus B, Kunz J and Radu E 2011 Phys.Rev.Lett. 106151104 [arXiv:1101.2868]

[485] Taveras V and Yunes N 2008 Phys. Rev. D78 064070 [arXiv:0807.2652]

[486] Alexander S and Yunes N 2008 Phys. Rev. D77 124040 [arXiv:0804.1797]

[487] Kanti P, Mavromatos N, Rizos J, Tamvakis K and Winstanley E 1996 Phys.Rev. D54 5049-5058 [arXiv:hep-th/9511071]

[488] Pani P and Cardoso V 2009 Phys.Rev. D79 084031 [arXiv:0902.1569]

[489] Ali-Haimoud Y and Chen Y 2011 Phys.Rev. D84 124033 [arXiv:1110.5329]

[490] Delsate T, Hilditch D and Witek H 2015 Phys.Rev. D91 024027 [arXiv:1407.6727] 
[491] Torii T, Yajima H and Maeda K i 1997 Phys.Rev. D55 739-753 [arXiv:gr-qc/9606034]

[492] Alexeev S and Pomazanov M 1997 Phys.Rev. D55 2110-2118 [arXiv:hep-th/9605106]

[493] Kanti P, Mavromatos N, Rizos J, Tamvakis K and Winstanley E 1998 Phys.Rev. D57 6255-6264 [arXiv:hep-th/9703192]

[494] Sago N, Nakano H and Sasaki M 2003 Phys. Rev. D67 104017 [arXiv:gr-qc/0208060]

[495] Konno K and Takahashi R 2014 Phys.Rev. D90 064011 [arXiv:1406.0957]

[496] Stein L C 2014 [arXiv:1407.0744]

[497] Stein L C 2014 Phys.Rev. D90 044061 [arXiv:1407.2350]

[498] Yunes N and Sopuerta C F 2008 Phys. Rev. D77 064007 [arXiv:0712.1028]

[499] Cardoso V and Gualtieri L 2009 Phys.Rev. D80 064008 [arXiv:0907.5008]

[500] Molina C, Pani P, Cardoso V and Gualtieri L 2010 Phys.Rev. D81 124021 [arXiv:1004.4007]

[501] Motohashi H and Suyama T 2012 Phys. Rev. D85 044054 [arXiv:1110.6241]

[502] Dyda S, Flanagan E E and Kamionkowski M 2012 Phys.Rev. D86 124031 [arXiv:1208.4871]

[503] Garfinkle D, Pretorius F and Yunes N 2010 Phys.Rev. D82 041501 [arXiv:1007.2429]

[504] Pani P, Macedo C F, Crispino L C and Cardoso V 2011 Phys.Rev. D84 087501 [arXiv:1109.3996]

[505] Ayzenberg D and Yunes N 2014 Phys.Rev. D90 044066 [arXiv:1405.2133]

[506] Ayzenberg D, Yagi K and Yunes N 2014 Phys.Rev. D89 044023 [arXiv:1310.6392]

[507] Lue A, Wang L M and Kamionkowski M 1999 Phys.Rev.Lett. 83 1506-1509 [arXiv:astro$\mathrm{ph} / 9812088]$

[508] Jackiw R and Pi S Y 2003 Phys. Rev. D68 104012 [arXiv:gr-qc/0308071]

[509] Satoh M, Kanno S and Soda J 2008 Phys.Rev. D77 023526 [arXiv:0706.3585]

[510] Satoh M and Soda J 2008 JCAP 0809019 [arXiv:0806.4594]

[511] Ali-Haimoud Y 2011 Phys. Rev. D83 124050 [arXiv:1105.0009]

[512] Ruffini R and Sasaki M 1981 Prog.Theor.Phys. 66 1627-1638

[513] Gair J R and Glampedakis K 2006 Phys.Rev. D73 064037 [arXiv:gr-qc/0510129]

[514] Hughes S A 2000 Phys.Rev. D61 084004 [arXiv:gr-qc/9910091]

[515] Ioka K and Taniguchi K 2000 Astrophys.J. 537327 [arXiv:astro-ph/0001218]

[516] Harko T, Kovacs Z and Lobo F S 2010 Class.Quant.Grav. 27105010 [arXiv:0909.1267]

[517] Kluzniak W and Abramowicz M A 2001 Acta Physica Polonica B 323605

[518] Abramowicz M A and Kluzniak W 2001 Astron.Astrophys. 374 L19 [arXiv:astro-ph/0105077]

[519] Schnittman J D and Bertschinger E 2004 Astrophys.J. 606 1098-1111 [arXiv:astro-ph/0309458]

[520] Amendola L, Charmousis C and Davis S C 2007 JCAP 0710004 [arXiv:0704.0175]

[521] Gonzalez Hernandez J I, Rebolo R and Casares J 2012 Astrophys.J. 744 L25 [arXiv:1112.1839]

[522] Arkani-Hamed N, Dimopoulos S and Dvali G R 1998 Phys. Lett. B429 263-272 [arXiv:hep$\mathrm{ph} / 9803315]$

[523] Arkani-Hamed N, Dimopoulos S and Dvali G R 1999 Phys. Rev. D59 086004 [arXiv:hep$\mathrm{ph} / 9807344]$

[524] Randall L and Sundrum R 1999 Phys. Rev. Lett. 83 3370-3373 [arXiv:hep-ph/9905221]

[525] Tanahashi N and Tanaka T 2011 Prog. Theor. Phys. Suppl. 189 227-268 [arXiv:1105.2997]

[526] 't Hooft G 1993 Dimensional reduction in quantum gravity Salamfest 1993:0284-296 pp 0284-296 [arXiv:gr-qc/9310026]

[527] Susskind L 1995 J. Math. Phys. 36 6377-6396 [arXiv:hep-th/9409089]

[528] Hawking S W, Hertog T and Reall H S 2000 Phys. Rev. D62 043501 [arXiv:hep-th/0003052]

[529] Garriga J and Sasaki M 2000 Phys. Rev. D62 043523 [arXiv:hep-th/9912118]

[530] Duff M J and Liu J T 2001 Class. Quant. Grav. 18 3207-3214 [Phys.Rev.Lett.85:2052-2055,2000] [arXiv:hep-th/0003237]

[531] Anderson P R, Balbinot R and Fabbri A 2005 Phys. Rev. Lett. 94061301 [arXiv:hep-th/0410034]

[532] Anderson P R and Fabbri A 2007 Phys. Rev. D75 044015 [arXiv:gr-qc/0612018]

[533] Shiromizu T and Ida D 2001 Phys. Rev. D64 044015 [arXiv:hep-th/0102035]

[534] Shiromizu T, Torii T and Ida D 2002 JHEP 03007 [arXiv:hep-th/0105256]

[535] Tanaka T 2004 [arXiv:gr-qc/0402068] 
[536] Grisa L and Pujolas O 2008 JHEP 06059 [arXiv:0712.2786]

[537] Hawking S W 1975 Commun. Math. Phys. 43 199-220 [Erratum-ibid.46:206-206,1976]

[538] Emparan R, Garcia-Bellido J and Kaloper N 2003 JHEP 01079 [arXiv:hep-th/0212132]

[539] Gregory R, Rubakov V A and Sibiryakov S M 2000 Class. Quant. Grav. 17 4437-4450 [arXiv:hepth/0003109]

[540] Hogan C J 2000 Phys. Rev. Lett. 85 2044-2047 [arXiv:astro-ph/0005044]

[541] Babichev E O, Dokuchaev V I and Eroshenko Y N 2013 Phys. Usp. 56 1155-1175 [Usp. Fiz. Nauk189,no.12,1257(2013)] [arXiv:1406.0841]

[542] Christodoulou D 1970 Phys. Rev. Lett. 25 1596-1597

[543] Belczynski K, Kalogera V and Bulik T 2001 Astrophys. J. 572 407-431 [arXiv:astro-ph/0111452]

[544] Gair J R et al. 2004 Class. Quant. Grav. 21 S1595-S1606 [arXiv:gr-qc/0405137]

[545] Psaltis D 2007 Phys. Rev. Lett. 98181101 [arXiv:astro-ph/0612611]

[546] Gnedin O Y, Maccarone T J, Psaltis D and Zepf S E 2009 Astrophys. J. 705 L168-L171 [arXiv:0906.5351]

[547] Simonetti J H, Kavic M, Minic D, Surani U and Vejayan V 2011 Astrophys.J. 737 L28 [arXiv:1010.5245]

[548] Weisberg J M and Taylor J H 2005 ASP Conf. Ser. 32825 [arXiv:astro-ph/0407149]

[549] Weisberg J M, Nice D J and Taylor J H 2010 Astrophys. J. 722 1030-1034 [arXiv:1011.0718]

[550] Ponce M, Palenzuela C, Barausse E and Lehner L 2015 Phys.Rev. D91 084038 [arXiv:1410.0638]

[551] Naf J and Jetzer P 2011 Phys.Rev. D84 024027 [arXiv:1104.2200]

[552] De Laurentis M and Capozziello S 2011 Astropart.Phys. 35 257-265 [arXiv:1104.1942]

[553] Blas D, Pujolas O and Sibiryakov S 2010 Phys.Rev.Lett. 104181302 [arXiv:0909.3525]

[554] Blas D, Pujolas O and Sibiryakov S 2011 JHEP 1104018 [arXiv:1007.3503]

[555] Barausse E and Sotiriou T P 2012 Phys.Rev.Lett. 109181101 erratum-ibid. 110, 039902 (2013) [arXiv:1207.6370]

[556] Wang A 2013 Phys.Rev.Lett. 110091101 [arXiv:1212.1876] 